\title{
WATER-LITHIUM BROMIDE DOUBLE-EFFECT \\ ABSORPTION COOLING ANALYSIS
}

Gary C. V1iet,

Michael B. Lawson, and

Rudolfo A. Lithgow

$$
\text { Center for Energy Studies }
$$

The University of Texas at Austin

December 1980

Final Report for

Contract: DE AC03-79SF 10540

(Multiple-Effect Absorption Cycle SoTar Cooling)

with the

U.S. Department of Energy 


\section{DISCLAIMER}

This report was prepared as an account of work sponsored by an agency of the United States Government. Neither the United States Government nor any agency Thereof, nor any of their employees, makes any warranty, express or implied, or assumes any legal liability or responsibility for the accuracy, completeness, or usefulness of any information, apparatus, product, or process disclosed, or represents that its use would not infringe privately owned rights. Reference herein to any specific commercial product, process, or service by trade name, trademark, manufacturer, or otherwise does not necessarily constitute or imply its endorsement, recommendation, or favoring by the United States Government or any agency thereof. The views and opinions of authors expressed herein do not necessarily state or reflect those of the United States Government or any agency thereof. 


\section{DISCLAIMER}

Portions of this document may be illegible in electronic image products. Images are produced from the best available original document. 


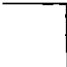

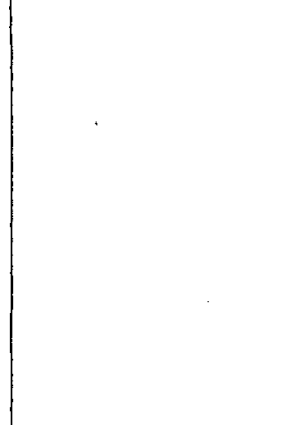




\section{ABSTRACT}

This investigation involved the development of a numerical model for the transient simulation of the double-effect, water-lithium bromide absorption cooling machine, and the use of the model to determine the effect of the various design and input variables on the absorption unit performance. The performance parameters considered were coefficient of performance and cooling capacity. The sensitivity analysis was performed by selecting a "nominal condition" and determining performance sensitivity for each variable with others held constant. The variables considered in the study include source hot water, cooling water, and chilled water temperatures; source hot water, cooling water, and chilled water flow rates; solution circulation rate; heat exchanger areas; pressure drop between evaporator and absorber; solution pump characteristics; and refrigerant flow control methods.

The performance sensitivity study indicated in particular that the distribution of heat exchanger area among the various (seven) heat exchange components is a very important design consideration. Moreover, it indicated that the method of flow control of the first effect refrigerant vapor through the second effect is a critical design feature when absorption units operate over a significant range of cooling capacity.

The model was used to predict the performance of the Trane absorption unit with fairly good accuracy. The dynamic model should be valuable as a design tool for developing new absorption machines or modifying current machines to make them optimal based on current and future energy costs. 


\section{TABLE OF CONTENTS}

Page

Abstract ........................ $i j i$

List of Tables........................... vii

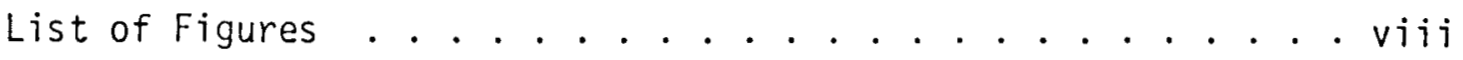

Nomenclature ............... . . . x

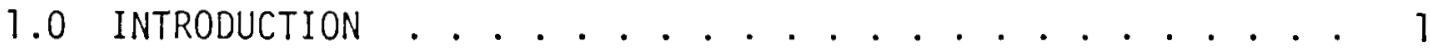

1.1 Background ................... 1

1.2 The Absorption Cool ing System . . . . . . . . . 2

1.3 Commercial Double-Effect Absorption Systems . . . . 6

1.4 Study Objectives............. 7

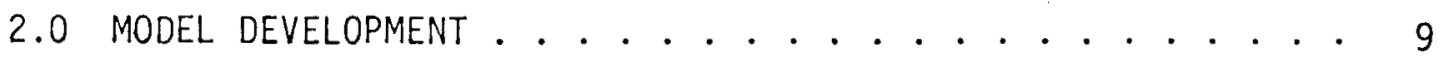

2.1 The Physical Model . . . . . . . . . . . . 9

2.2 The Computer Model . . . . . . . . . . . . 11

2.2.1 Variables ................ . 12

2.2.2 Method of Solution ........... 12

2.3 Component Models............. . . 15

2.3.1 The First Generator . . . . . . . . . 15

2.3.2 The Second Generator . . . . . . . . 19

2.3 .3 The Condenser............ . . 22

2.3 .4 The Evaporator . . . . . . . . . 24

2.3 .5 The Absorber ........... 26

2.3.6 The Heat Exchangers .......... 30

2.4 Mode1 Limitations .............. . . 31

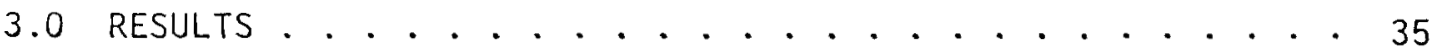

3.1 Method of Analysis . . . . . . . . . 35

3.2 The Nominal Condition ........... 35

3.3 Individual Sensitivity Scans .......... 38

3.3.1 Scans Based on the Nominal Condition .... . 41

(a) Source Hot Water Temperature . . . . . 41

(b) Cooting Water Temperature ....... 43

(c) Chilled Water Temperature . . . . . . 43

(d) Source Hot Water. Flow Rate...... 43

(e) Cooling Water Flow Rate........ 47

(f) Chilled Water Flow Rate . . . . . . . 47

(g) Solution Circulation Rate ....... 47

(h) Areas of Generators, Condenser, Evaporator, and Absorber .......... 51

(i) Heat Exchanger Areas ........ . . 51 
3.3.2 Effect of Orifice Flow Control ...... . 59

3.3.3 Effect of Variable Solution Flow Rate .... 63

3.3.4 Effect of Combined Orifice Flow Control and

Variable Solution Flow Rate....... 63

3.4 Performance Maps ... . . . . . . . . . . 68

3.5 Comparison with Trane Two-Stage Absorption Unit

Performance ............ . 73

3.6 Cost Projections ............... 75

4.0 SUMMARY . . . . . . . . . . . . . . . . . . 78

4.1 Conclusions ................... . . 78

4.2 Recommendations ............ 80

Appendix A: COMPUTER PROGRAM LIBR . . . . . . . . 83

Appendix B: HEAT TRANSFER COEFFICIENTS FOR WATER-LITHIUM

BROMIDE SOLUTIONS AS FUNCTIONS OF HEAT FLUX,

PRESSURE, AND CONCENTRATION ....... 107

Appendix C: EQUILIBRIUM CHART FOR WATER-LITHIUM BROMIDE

SOLUTIONS ................ 111

Appendix D: THE NOMINAL CONDITION . . . . . . . 113

References................. . 1715 


\section{LIST OF TABLES}

Table

Page

2.1 Variation in Fluid Specific Volume over Extreme Operating Range ............. . 34

3.1 Nominal Conditions and Parameter Ranges . . . . . 36 


\section{LIST OF FIGURES}

Figure

Page

1.1 Single-Effect Absorption System . . . . . . . . . 3

1.2 Double-Effect Absorption System . . . . . . . . 5

2.1 Wegstein Solution Method . . . . . . . . . 14

2.2 Schematic Model .............. 16

3.1a The Nominal Condition .. . . . . . . . . . . 39

3.1b Two-Stage Water-Lithium Bromide Cycle for Operation at the Nominal Condition ............ . 40

3.2a Effect of Source Hot Water Temperature on Performance 42

3.2b Effect of Cooling Water Temperature on Performance . 44

3.2c Effect of Chilled Water Exit Temperature on

Performance............... . . 45

3.3a Effect of Source Hot Water Flow Rate on Performance. 46.

3.3b Effect of Cooling Water Flow Rate on Performance . . 48

3.3c Effect of Chilled Water Flow Rate on Performance . 49

3.4 Effect of Solution Flow Rate on Performance. . . . . 50

3.5a Effect of Generator 1 Area on Performance . . . . . 52

3.5b Effect of Generator 2 Area on Performance . . . . . 53

3.5c Effect of Condenser Area on Performance . . . . . . 54

3.5d Effect of Evaporator Area on Performance . . . . . 55

3.5e Effect of Absorber Area on Performance . . . . . . 56

3.5f Effect of Heat Exchanger 1 Area on Performance . . 57

3.5g Effect of Heat Exchanger 2 Area on Performance . . 58

3.6 Effect of Evaporator-Absorber Flow Coefficient on

Performance ................. 60

3.7 Effect of Orifice Flow Control on Performance . . . 62 
$\underline{\text { Figure }}$

Page

3.8a Effect of Centrifugal Pump I on Performance . . . . . 64

3.8b Effect of Centrifugal Pump II on Performance . . . 65

3.9 Pump I and II Characteristics .......... 66

3.10a Effect of Orifice Flow Control with Centrifugal Pump I 67

3.10b Effect of Orifice Flow Control with Centrifugal Pump II . . . . . . . . . . . . . . . 69

3.11 a Performance for $40^{\circ} \mathrm{F}$ Exit Chilled Water . . . . . 70

3.11b Performance for $50^{\circ} \mathrm{F}$ Exit Chilled Water . . . . . 71

3.11c Performance for $60^{\circ} \mathrm{F}$ Exit Chilled Water . . . . . . 72

3.12 Comparison of Predicted and Trane Results . . . . . 74

3.13 Comparison of Cost per Ton versus Tonnage for WaterLithium Bromide Absorption Systems. . . . . . . 76 


\section{NOMENCLATURE}

\section{Variable Description}

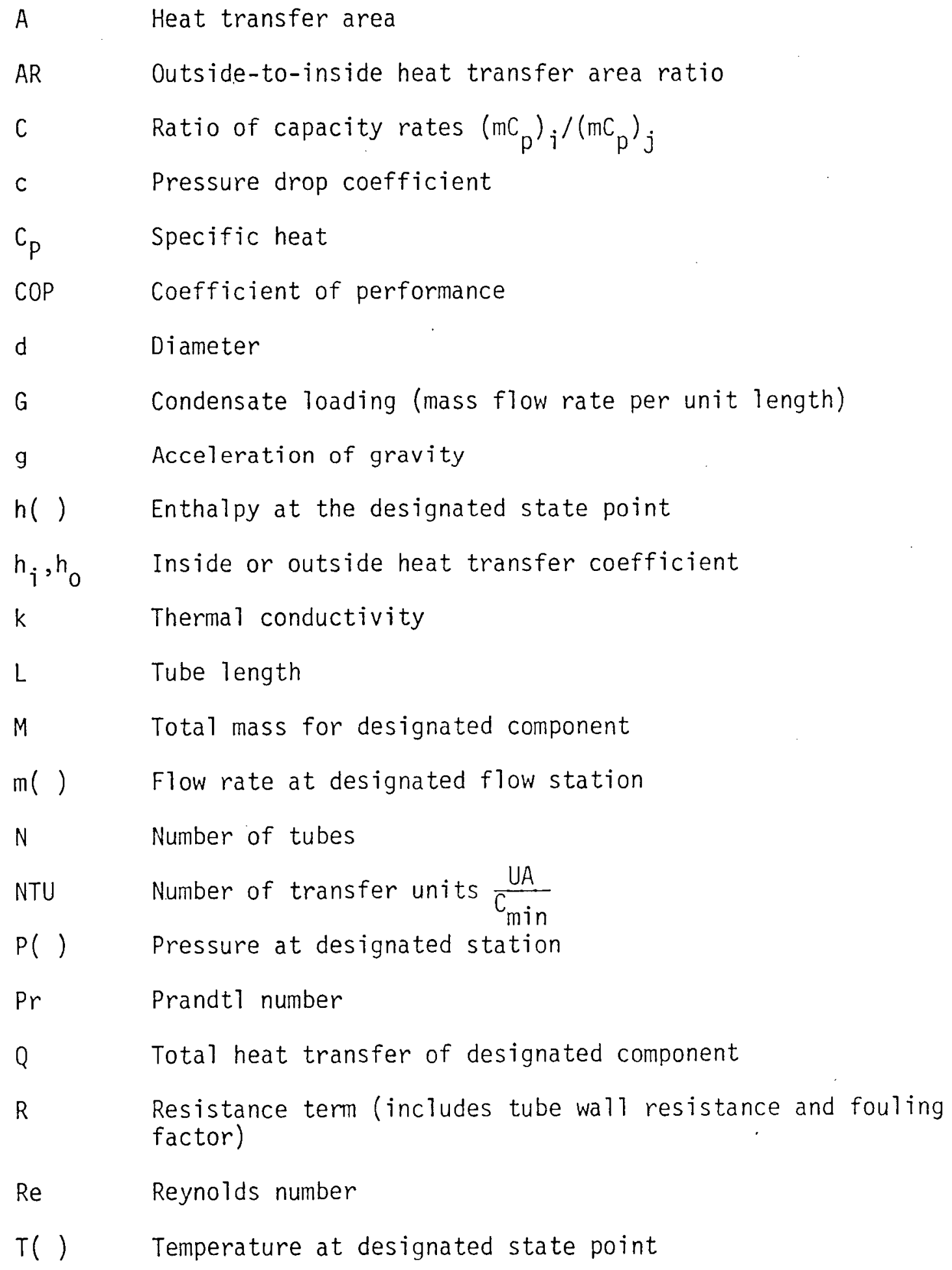


Variable Description

T12 Equilibrium temperature at state point 12

$T_{C} \quad$ Cooling water temperature entering absorber

$T_{C}{ }^{\prime} \quad$ Absorber exiting cooling water temperature

$T_{C} " \quad$ Condenser exiting cooling water temperature

$T_{e} \quad$ Chilled water temperature exiting evaporator

$T_{e}{ }^{\prime} \quad$ Temperature of water entering the evaporator

$T_{S} \quad$ Source hot water entering temperature

$T_{S}^{\prime} \quad$ Source hot water exiting temperature

U Overall heat transfer coefficient

$X($ ) Concentration of solution at designated state point

$\varepsilon \quad$ Heat exchanger effectiveness

$\mu \quad$ Viscosity

$\checkmark \quad$ Kinematic viscosity

$\rho \quad$ Density

$\theta \quad$ Time

Subscript Description

A Absorber

C Condenser

ch chilled water

c Cooling water

E, e Evaporator

G1 First generator

G2 Second generator

min Refers to the minimum value 
Subscript Description

nom Refers to the nominal condition

s Refers to the source hot water supply 


\subsection{INTRODUCTION}

\subsection{Background}

Considerable research is currently being done to improve available methods and to find new or alternative methods for air conditioning buildings. One such concept is double-effect absorption cooling. The particular advantage of the double-effect concept over single-effect is the possibility of considerably improved coefficients of performance (COP), of course at the expense of a higher required input temperature.

The current project arose from an interest in examining the potential of the double-effect absorption cooling concept for applications involving solar, geothermal, or waste energy input. Initially the interest was focused on the possible advantages of separating the "regeneration" and "heat pumping" portions of the cycle and inserting refrigerant and solution storage reservoirs between. The purpose of separation would be both to provide chemical storage and possibly to permit improved operation of each of these functions if they were allowed to operate independently. Subsequently the effort was directed primarily toward developing a dynamic simulation program to model the waterlithium bromide system and to use the model to develop parametric performance data for the system. Secondarily the project examined the cost of double-effect units. The interest resulted in a contract between the U.S. Department of Energy and The University of Texas at Austin, Center for Energy Studies: "Multiple-Effect Absorption Cycle Solar Cooling," Contract DE AC03-79SF10540, for the period September 1, 1979, to November 30, 1980. 


\subsection{The Absorption Cooling System}

The absorption process was first demonstrated by Michael Faraday in 1824 using ammonia as the refrigerant and silver chloride as the absorbent [1]. Since that time, many absorption systems using various refrigerant/absorbent combinations have been developed. Auh provides an overview of absorption cooling [2].

The absorption process can be compared to the vapor compression system in that the process of mechanical compression of the refrigerant gas is replaced by the absorption of the gas into a liquid (solution), followed by pumping the liquid to the required pressure where the refrigerant vapor is liberated from the solution by application of heat. Figure 1.1 illustrates the flow of a typical single-effect absorption system.

Refrigerant vapor (water in the water-lithium bromide system) produced in the evaporator is absorbed in strong (in lithium bromide) solution in the absorber, thus maintaining the low pressure and low temperature required in the evaporater. Dilute solution at low pressure in the absorber is pumped through a heat exchanger to a higher pressure in the generator. In the generator, this solution is boiled and the refrigerant is released, leaving a solution that is stronger in the absorbent (1ithium bromide in the water-lithium bromide system). The strong solution is then returned through the other side of the heat exchanger and back to the absorber.

The heat exchanger has two functions: to heat the solution going to the generator to reduce the source heat requirements of the generator; and to cool the strong solution before returning to the absorber, making it a more efficient absorbent and also reducing the cooling 


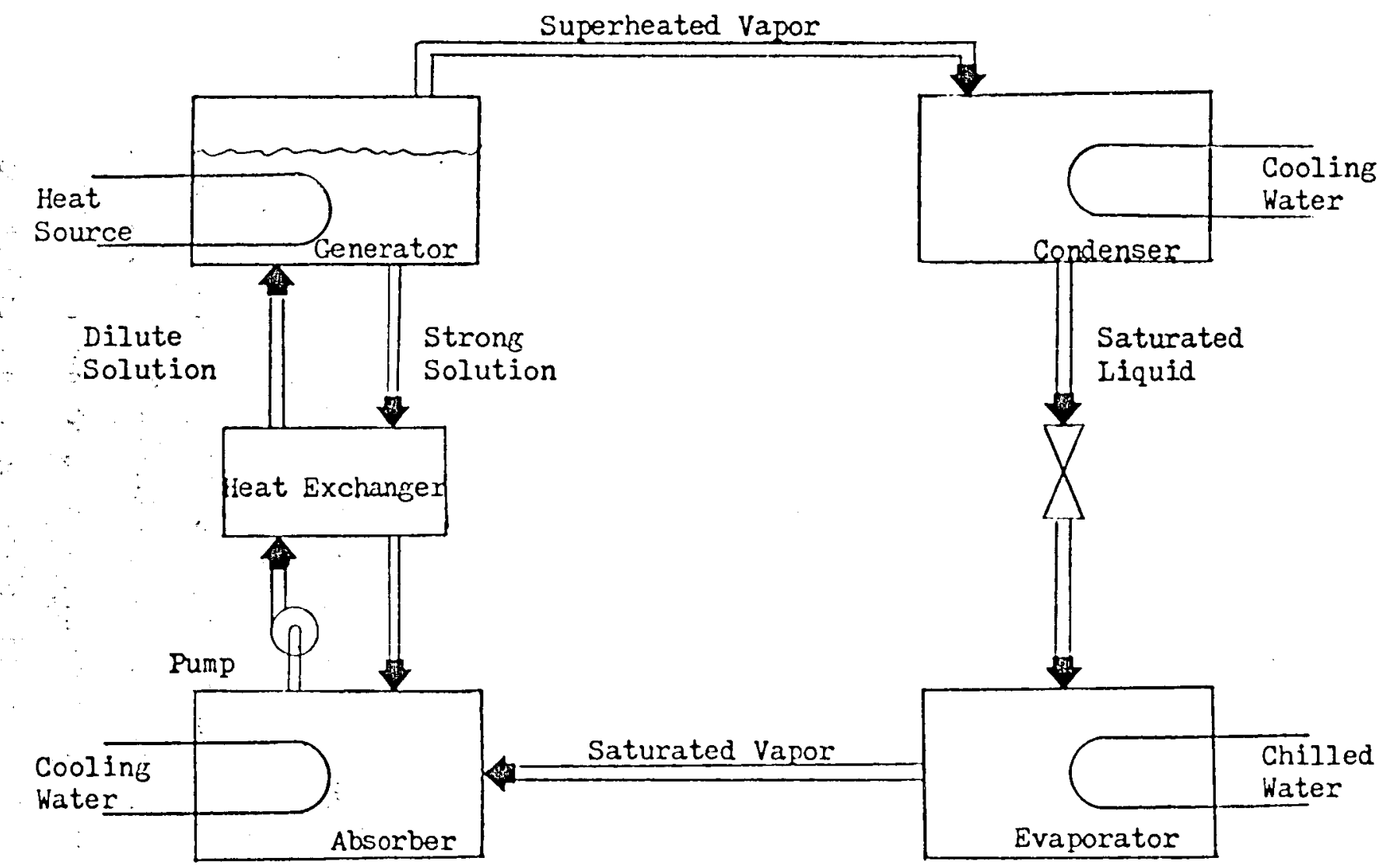

Figure 1.1 SINGLE-EFFECT ABSORPTION SYSTEM 
demand of the absorber. These three components, together with the solution pump, accomplish the task of the compressor in a conventional vapor compression system.

The remaining part of the cycle is almost identical to a vapor compression cycle. Superheated vapor produced in the generator is cooled and condensed in the condenser to a saturated liquid state. This liquid refrigerant is then throttled across a valve into the evaporator, where upon evaporation at low pressure it absorbs energy from the water being chilled. It leaves as nearly saturated vapor and flows to the absorber, where it is absorbed into the solution. The required low pressure and low temperature in the evaporator are made possible by the absorption process.

The coefficients of performance of single-effect systems are less than unity because energy added in the generator must necessarily overcome both the heat of solution and heat of evaporation, but only the heat of evaporation may be used for cooling in the evaporator. Considering other situations that are not ideal, the maximum COP for singleeffect absorption cycles is typically about 0.7 , with actual cycles operating under steady-state conditions exhibiting COPs near 0.6.

In the single-effect cycle the heat delivered to the generator is rejected in the condenser. However, if the vapor produced in the generator is condensed instead by transferring its energy to solution leaving the generator at lower pressure, additional refrigerant vapor can be produced, thus resulting in a higher cycle COP.

Figure 1.2 shows this so-called double-effect absorption system, which utilizes two generators. The superheated refrigerant of the first generator is used as a heat source in the lower pressure second generator, to produce additional refrigerant in the second generator. 


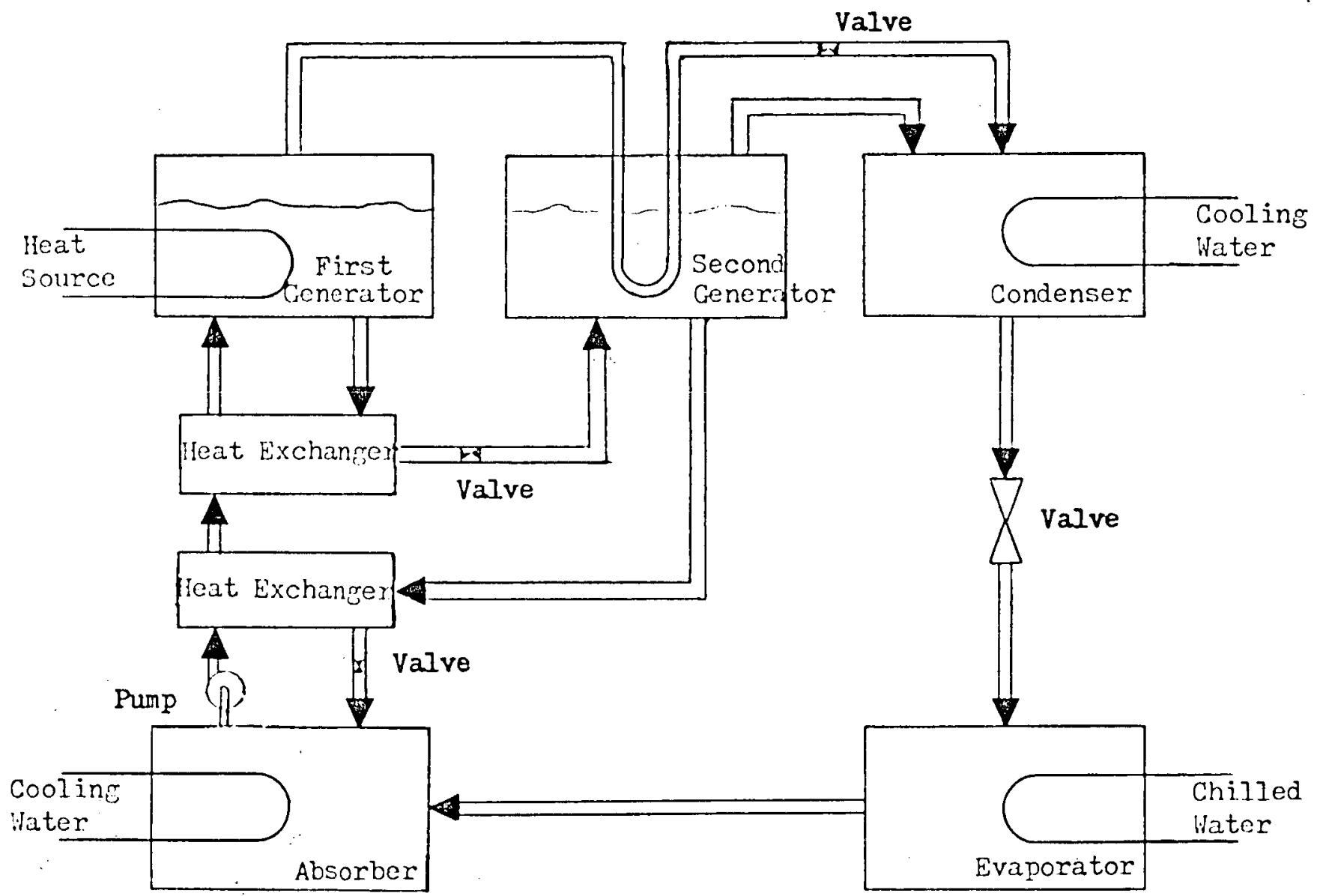

Figure 1.2 DOUBLE-EFFECT ABSORPTION SYSTEM 
Ideally, the first generator's refrigerant will leave the second generator as saturated liquid at the pressure of the first generator. The salient feature of the double-effect concept is that a high COP is achieved, since the source energy produces more refrigerant vapor. Thus, a larger cooling effect is achieved in the evaporator for the same source energy, compared to a single-effect unit. The water cooling load in the double-effect system is also lower per ton of cooling than that of the single-effect system. The disadvantages are that higher source temperatures are needed in the double-effect system, a second heat exchanger is required, and the system is somewhat more complex. However, with markedly increasing energy costs, the potentially greater COP makes the double-effect system very attractive.

\subsection{Commercial Double-Effect Absorption Systems}

There has been only limited manufacture of double-effect absorption systems, all of which use the water-lithium bromide system. The first prototype double-effect unit was developed by Southwest Research Institute in 1956-58 with funding from the American Gas Association [3, 4]. Later, the Iron Fireman Company of Cleveland, Ohio, purchased the manufacturing rights and began production of the "Iron Fireman" unit in 1963. This gas-fired unit had a capacity of 15 tons and a measured gross COP of about 0.9 to 1.0 at design capacity. On the basis of the net heating value of the gas, this should result in an actual COP of 1.2 to 1.3. Only a few of these units were manufactured, and it is not known if any are still in operation. The unit is no longer in production. 
The Trane Company, in 1973, introduced its double-effect absorption units with capacities between 385 and 1,060 tons. These units have COPs in the range of 0.9 to 1.0 at design capacity. The Sanyo Company of Japan had previously built a line of double-effect units; however, the line has been discontinued. Yazaki, also of Japan, has announced it will introduce a gas-fired unit of 20 tons in the fall of 1980.

Although several double-effect cooling systems have been designed, the designs do not appear to be as efficient as they should be, probably because they were designed in the time of cheap energy. Preliminary investigations of double-effect systems indicate potential COPs for the water-lithium bromide systems to be well above those achieved in commercial units [5].

\subsection{Study Objectives}

The objectives of this study were threefold:

(a) To develop a dynamic numerical computer model to simulate the water-lithium bromide double-effect absorption cooling system, including all major components and allowing flexibility in operation.

(b) To develop with the model, performance curves for the unit, indicating how coefficient of performance and capacity are affected by its several design and input variables. These variables include source hot, cooling, and chilled water temperatures and flow rates; solution circulation rate; and heat exchanger areas. In addition, other factors such as: pressure drop between evaporator and absorber, solution pump 
characteristics, control of refrigerant flow from the first generator, and limits of operation were to be examined.

(c) To assess projected costs for intermediate-size double-effect units.

These results will hopefully be used as the basis for designing new, more efficient double-effect water-lithium bromide absorption units. They can also be used to estimate the reduced capacity of units operating at off-design conditions. The computer program itself should be a valuable design tool for optimizing unit performance, and in conjunction with a more general systems program could be used to predict and optimize the performance of cooling units with widely varying heat input sources, such as solar or geothermal energy or waste heat.

This study shows that double-effect absorption units can be operated. at lower temperatures than are generally reported in the 7 iterature, with little effect on COP but with sacrifice in capacity. In addition, the actual cooling unit performance should be greater than that of available commercial units. Finally, it is hoped that this report will reveal that double-effect machines are a viable alternative to other cooling systems and can operate over a wide range of conditions in many more applications than those in which they are presently being used. 


\subsection{MODEL DEVELOPMENT}

\subsection{The Physical Model}

The basic system configuration is shown schematically in Figure 1.2. Mass and thermal storage are allowed in the first generator, the second generator, the condenser, and the absorber; however, the evaporator has no mass storage capability. The first and second generators have fixed mass levels, but the condenser and absorber masses are allowed to vary. The variable masses in these components allow the concentration levels in the unit to adjust as required to satisfy mass, energy, and equilibrium criteria. In specifying many of the details of the design that are discussed below, the Trane double-effect units have been used as guides.

The first generator is a vessel containing water-lithium bromide solution which is heated by heat exchanger tubes with a heating fluid (water in the results which follow), thereby generating refrigerant (water) vapor from the solution. In the second generator the slightly superheated water vapor produced by the first generator is condensed in heat exchanger tubing to generate additional refrigerant (water) vapor from solution. The solution in the second generator is weaker (in refrigerant) than in the first but exists at a lower pressure because it is throttled after leaving the first generator. Constant masses in each of the generators are maintained by float control valves.

The condenser is configured so that the cooling water is carried in horizontal tubes and condensation of the refrigerant (water) vapor occurs on the outside of the tubes. A storage trough for liquid refrigerant collection is incorporated below the condenser tubes in the condenser design. 
The evaporator heat exchanger is assumed to be a horizontal tubetype configuration where the chilled water is cooled inside the tubes and the liquid refrigerant from the condenser is sprayed over the outside of the tubes, where it is evaporated.

The absorber is configured so that solution returning from the second generator is sprayed over horizontal tubes through which the cooling water flows. This solution absorbs water vapor coming from the evaporator, thus maintaining a low evaporator pressure. Solution, rich in refrigerant falling from the tubes, is collected in the bottom of the absorber. An option in the model allows solution from the absorber basin to be recirculated and mixed with the solution from the second generator before being sprayed over the absorber tubes. Solution from the absorber is pumped through two preheating heat exchangers on the way to the first generator.

While shown as individual components, the second generator and the condenser are commonly housed in a single container. Similarly, the evaporator and absorber are often combined. The reason is that the evaporator and absorber operate at essentially the same low pressure, 5 to $8 \mathrm{~mm}$ of mercury, while the condenser and second generator both operate in the range of 50-80 mm of mercury, which is also quite low. All four components operate wel1 below atmospheric pressure.

The two liquid-liquid heat exchangers are of the shell-and-tube type. Dilute (in refrigerant) solutions flow through the tube sides and the strong (in refrigerant) solutions flow through the shells. These heat exchangers are assumed to contain negligible mass.

The main solution circulation pump can be either a centrifugal pump, for which flow is a function of the pressure head or, possibly, a 
constant displacement pump, which maintains constant flow rate at any pressure differential. In the performance analyses that follow, emphasis is placed on the case of constant solution circulation rate. However, comparisons are made of the effect of centrifugal pumps with different characteristics. Pumps for recirculation of liquid over the tubes in the evaporator and absorber are not considered. In the evaporator, however, the evaporation coefficient could be adjusted to account for recirculation if desired. The absorber includes the option for recirculation of solution, though recirculation was not used in this study. The effect of recirculation in the absorber would be to change the states (temperature and concentration) of the solution distributed over the tubes and also the nature of the fluid mechanics and heat and mass transfer for the falling film.

Another important choice in the physical configuration of the system is the mechanism for flow control of the refrigerant from the first generator through the second generator to the condenser. This flow can be controlled so that the fluid leaving the second generator is only liquid, by incorporating a float valve that does not allow vapor to pass. The other alternative is a fixed area orifice-type of restriction which is commonly used in commercial absorption machines built today. The float valve design is used in virtually all of the analyses since it maximizes performance. However, the relative performance of systems with an orifice control is also examined.

\subsection{The Computer Model}

The numerical computer model for this investigation of the waterlithium bromide double-effect absorption cooling system was developed in 
the FORTRAN Tanguage for use on the CDC CYBER 170/750. computer at The University of Texas. Appendix A contains the complete computer program listing. Much of the preliminary work in developing the program was done by Rodolfo A. Lithgow [6].

Basically, the model requires the solution of a large set of nonlinear equations. The solution involves two methods. The first is an iterative method called the "Wegstein Method for Algebraic Convergence." The second is the integration of a computed differential which is determined from the convergence step. Both methods are described by Franks [7].

\subsubsection{Variables}

Three kinds of variables--two algebraic forms and one derivative form--are included in the program. The first type is one which is explicitly defined by an algebraic expression. Implicit variables, determined from an equation in which the variables appear on both sides of the equation, are the second type. The third is an integrated variable. For each integrated variable, a derivative of that variable with respect to time is formed from one of the governing equations (mass of energy), and the variable is integrated to obtain a new value for the next time step.

\subsubsection{Method of Solution}

In mathematically defining a double-effect water-lithium bromide absorption system, a geometry must first be selected. Then equations for energy, mass, mass species, and state are written for each component in the system. 
The equations are arranged sequentially so that variables solved for in one equation can be used in the solution of the next equation. This is continued to a point where the next equation is implicit rather than explicit. This chain of equations defines an iterative loop to be solved by the "Wegstein Method." Optimization and selection of these loops are discussed by Himmelblau and Bischoff [8].

The Wegstein method is carried out in the program by the subroutine CONV, which is listed in Appendix A. Its function can be explained graphically by using Figure 2.1. The desired solution is shown by the intersection of the function and the $45^{\circ}$ diagonal line, where $X$-calculated $(X C)$ is equal to $X$. To start, an assumed value, $X 1$, is chosen for $X$. Using this value for $X$ in the right-hand side of the equation results in a computed value of $X C 1$ (point 1). The value $X C l$ is then used as a value, $X 2$, for the variable $X$ resulting in a computed value, $X C 2$, at point 2. A straight 1 ine through points 1 and 2 is extrapolated to intersect with the $45^{\circ}$ diagonal at point 3 which gives the new value for $x$. When this value is used in the solution of the equation as above, the value at point 4 is found. Then a line using points 2 and 4 is constructed. The intersection of this line and the $45^{\circ}$ diagonal gives a more accurate value of $x$. This process is continued until the desired accuracy is obtained.

Since in this method the equations must be grouped together in an algebraically logical manner rather than a functionally logical manner, extreme care must be taken to insure that all functional requirements are met. However, when the problem is properly defined and constrained, this technique for solution is rapid. 


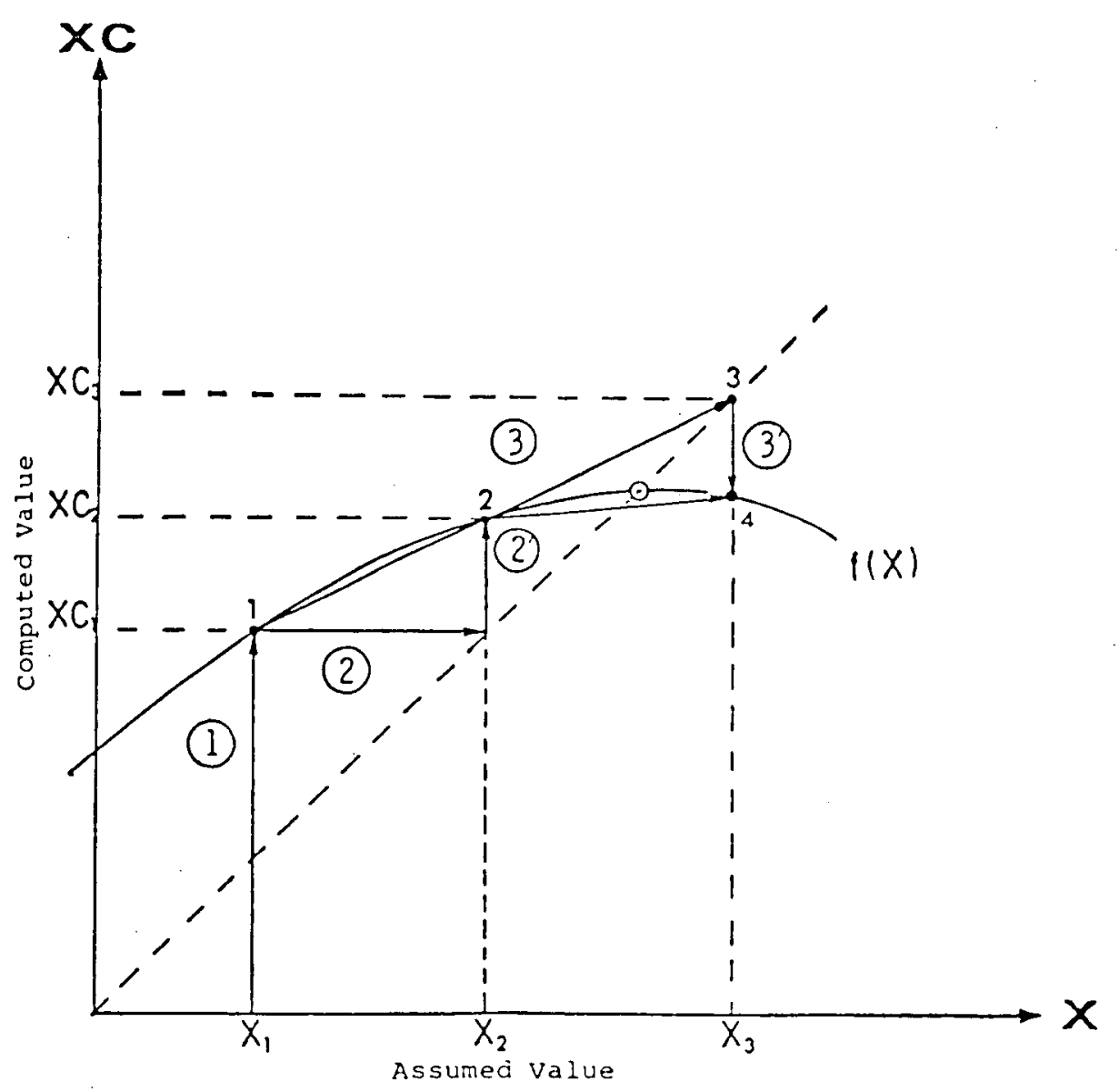

Figure 2.1 WEGSTEIN SOLUTION METHOD 


\subsection{Component Models}

Figure 2.2 shows a schematic of the system for which the state points correspond to those in the computer model. In the model, the following units are used:

- Temperatures, ${ }^{\circ} \mathrm{F}$

- Pressures, $1 \mathrm{bf} / \mathrm{in}^{2}$

- Flows, $\mathrm{lb} / \mathrm{hr}$

- Concentrations, lb LiBr/lb solution

- Linear dimensions, $\mathrm{ft}$

- Areas, $\mathrm{ft}^{2}$

- Masses, ib

- Times, $\mathrm{hr}$

\subsubsection{The First Generator}

The first or high-temperature generator is modeled as a pool of water-lithium bromide solution heated by a submerged tube bundle through which pressurized hot water is circulated. For this component, a transport equation can be written in the following form:

$Q_{G]}=m_{s}\left(T_{S}-T_{S}{ }^{\prime}\right)=(U A) \frac{\left[T_{S}-T(1)\right]-\left(T_{S}{ }^{\prime}-T 12\right)}{\ln \frac{T_{S}-T(1)}{T_{S}{ }^{\prime}-T 12}}$.

Note that specific heat here is inferred to be 1.0 since the heating fluid is water. In the computer program, the equation is rewritten in an implicit form so that $T_{S}{ }^{\prime}$ is the unknown. The overall heat transfer coefficient-area product, UA, is obtained by

$$
(U A)_{G l}=\frac{A_{G l}}{\frac{A R}{h_{i}}+\frac{1}{h_{0}}+R}
$$




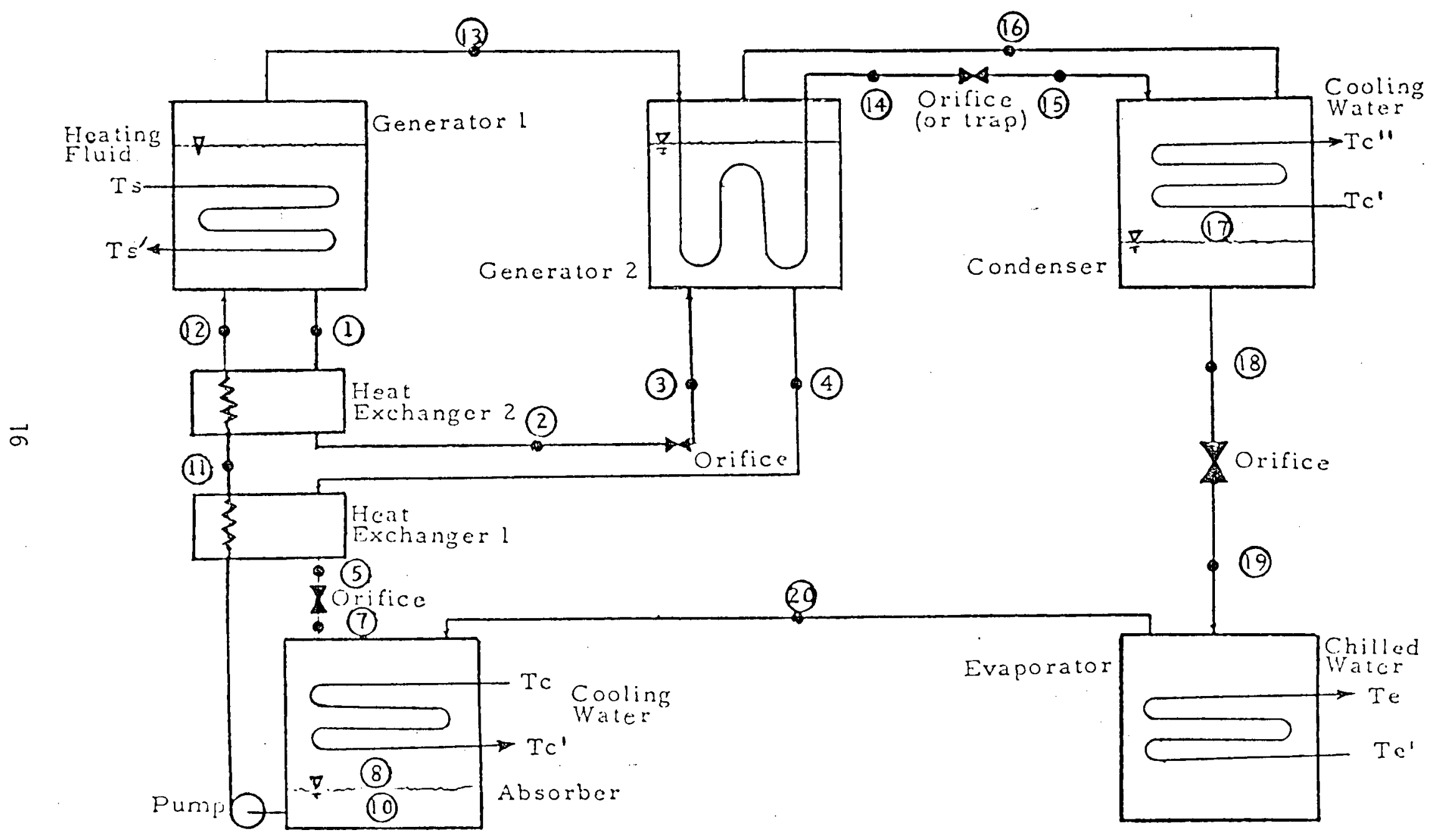

Figure 2.2 SCHEMATIC OF PHYSICAL MODEL 
where

$$
h_{i}=\frac{0.023 \cdot k \cdot \operatorname{Re}^{0.8} \operatorname{Pr}^{0.3}}{d},
$$

which is the commonly used expression for a fluid in turbulent flow being cooled inside tubes, for example, [9]. The outside heat transfer coefficient is given by

$$
h_{0}=f(Q / A, P, X)
$$

The particular functional relationship was determined from the experimental results of Minchenko and Firsova [10] for water-lithium bromide, and is plotted in English units in Appendix B. Subroutine HOGEN shown in Appendix A gives the curve fitted equations used in the program to calculate $h_{0}$. The term $A R$ is the outside to inside heat transfer area ratio, and $R$ is the combined tube wall/fouling factor resistance. (AR is set to 1.08 throughout the program, and the resistance is set to zero for this study.)

An energy balance equation written for the generator results in a time derivative for $T(1)$ :

$$
\frac{d}{d \theta}\left[M_{G 1} C_{p} T(1)\right]=m(12) h(12)-m(1) h(1)-m(13) h(13)+Q_{G 1}
$$

which simplifies to

$$
\frac{d T(1)}{d \theta}=\frac{m(12) h(12)-m(1) h(1)-m(13) h(13)+Q_{G I}+M_{G I} T(1) \frac{d C_{p}}{d X} \frac{d X}{d \theta}}{M_{G l} C_{p}}
$$


Approximating the value for the variation of specific heat with respect to concentration as 0.7157 (derived from data in reference [11]) gives the final form:

$\frac{d T(1)}{d \theta}=\frac{m(12) h(12)-m(1) h(1)-m(13) h(13)+Q_{G 1}+0.7157 M_{G 1} T(1) \frac{d X}{d \theta}}{M_{G 1} C_{p}}$

This expression (Equation 7) is linearly integrated after all the convergence criteria have been met. $T 12$, the remaining variable in the log-mean temperature expression (Equation 1), is determined by the equilibrium condition at state point 12 and is given by

$$
T 12=f[P(12), X(12)] .
$$

An equilibrium chart to evaluate this function is given in Appendix C. The subroutine TEMPSOL, shown in Appendix A, presents the numerical method for evaluating the function in the program. It should be noted that in order to use the log-mean temperature approach, state point 12 must be at the equilibrium condition, or else the log-mean approach is a poor approximation. The actual temperature at state point 12, $T(12)$, is not the equilibrium temperature, but a better representation for the heat transfer is obtained when the equilibrium temperature $\mathrm{T} 12$ is used in the log-mean temperature difference expression than the actual temperature $T(12)$.

The actual temperature is given by an energy balance on heat exchanger 2: 


$$
T(12)=T(11)+\frac{m(1) C_{p}[T(1)-T(2)]}{m(12) C_{p}}
$$

where the specific heats are each functions of the concentrations of the two streams.

In addition to the energy and transport equations, a species mass balance equation for the first generator is obtained by writing a concentration derivative for $x(1)$ :

$$
\frac{d}{d \theta}\left[M_{G T} \times(1)\right]=m(12) \times(12)-m(1) \times(1)
$$

which simplifies to

$$
\frac{\mathrm{d} X(1)}{\mathrm{d} \theta}=\frac{\mathrm{m}(12) \times(12)-\mathrm{m}(1) \times(1)}{M_{G 1}}
$$

since the generator mass is constant.

Finally, the overall mass balance on the first generator is given by

$$
m(1)=m(12)-m(13)
$$

\subsubsection{The Second Generator}

As in the first generator, the second or low-temperature generator is modeled as a pool of water-lithium bromide solution with submerged heat exchanger tubes. Refrigerant vapor from the first generator condenses on the insides of these tubes. 
The transport equation for the second generator is:

$$
Q_{G 2}=(U A)_{G 2} \frac{T(4)-T(3)}{\ln \left[\frac{T(14)-T(3)}{T(74)-T(4)}\right]}
$$

where the overall heat transfer coefficient is obtained as it was for the first generator (Equation 2). The inside condensing heat transfer coefficient is specified by:

$$
h_{i}=1.51\left(\frac{4 G}{\mu}\right)^{-1 / 3}\left(\frac{k^{3} p^{2} g}{k^{2}}\right)^{1 / 3}
$$

This inside heat transfer coefficient is based on an expression from Kern for condensing vapors [12]. For vapors condensing inside horizontal tubing, the loading $G$ in the above equation is defined as:

$$
G=\frac{2 m}{L N}
$$

where $\mathrm{m}, \mathrm{L}$, and $\mathrm{N}$ are the mass flow rate, tube length, and number of tubes, respectively.

The outside heat transfer coefficient is calculated as it was for the first generator (Equation 4), using subroutine HOGEN, which is based on the boiling heat transfer coefficient data of Minchenko and Firsova.

When an energy balance is written for the second generator, the following time derivative for $T(4)$ results:

$$
\frac{d}{d \theta}\left[M_{G 2} C_{p} T(4)\right]=m(3) h(3)-m(16) h(16)-m(4) h(4)+Q_{G 2}
$$


which simplifies to

$\frac{d T(4)}{d \theta}=\frac{m(3) h(3)-m(16) h(16)-m(4) h(4)+Q_{G 2}+0.7157 M_{G 2} T(4) \frac{d X(4)}{d \theta}}{M_{G 2} C_{p}}$

This is then integrated to obtain a value for $T(4)$.

The temperature (TI4) is forced to the saturated condition

$$
T(14)=f[P(14)]
$$

by the float flow control feature. The actual functional relationship is given in subroutine TVSP, which is available in Appendix $A$. When an orifice-type of control is used, Equation 18 is appropriate if complete condensation does not occur, but if complete condensation and subcooling occur, this is accounted for correctly in the program.

The solution at state point 3 , entering the second generator, is assumed to be in equilibrium. Thus, T(3) in the log-mean temperature difference (Equation 13) is derived as:

$$
T_{7}(3)=f[P(3), X(3)]
$$

The overall mass balance on the second generator is given by:

$$
m(4)=m(3)-m(16) .
$$

The species mass balance is determined by writing a time derivative for the concentration at state point 4 as follows: 


$$
\frac{d}{d \theta}\left[M_{G 2} \times(4)\right]=m(3) \times(3)-m(4) \times(4)
$$

which simplifies to

$$
\frac{d X(4)}{d \theta}=\frac{m(3) \times(3)-m(4) X(4)}{M_{G 2}}
$$

\subsubsection{The Condenser}

The condenser is modeled assuming the refrigerant vapor condenses on the outside of the tubes through which the cooling water flows. A log-mean temperature difference equation is written as:

$$
Q_{C}=m_{C}\left(T_{C}^{\prime \prime}-T_{C}^{\prime}\right)=(U A) \frac{T_{C}^{\prime \prime}-T_{C}^{\prime}}{\ln \left[\frac{T(17)-T_{C}}{T(17)-T_{C}^{\prime \prime}}\right]} .
$$

This can be simplified to

$$
T_{C}^{\prime \prime}=T(17)-\frac{\left[T(17)-T_{C}^{\prime}\right]}{\exp \frac{(U A)_{C}}{m_{C}}}
$$

which is the form used in the program. The overall heat transfer coefficient is determined as it was for the generators (Equation 2). On the inside, the heat transfer coefficient is again based on the turbulent correlation for flow inside tubes [9]:

$$
h_{i}=\frac{0.023 \cdot k \cdot R e^{0.8 P^{0.4}}}{d}
$$


(Note that for the case of fluid being heated, the Prandtl number exponent is 0.4.) The outside condensing heat transfer coefficient is obtained using a similar expression to that used for the inside heat transfer coefficient equation from the second generator (Equation 14), except that since the fluid is condensing on the outside of horizontal tubes, the loading is changed to

$$
G=\frac{m}{L(N)^{2 / 3}} .
$$

as suggested by Kern [12].

Two energy balance equations can be written for the condenser. One is for the cooling tube bundle which is located above the refrigerant storage trough, and the other is for the trough. For the tube bundle:

$$
Q_{C}=m(16)[h(16)-h(17)]+m(15)[h(15)-h(17)]
$$

For the trough, the energy balance results in the following time differential for $T(18)$ :

$$
\frac{d}{d \theta}\left[M_{C} C_{p} T(18)\right]=[m(15)+m(16)] h(17)-m(18) h(18)
$$

which simplifies to

$$
\frac{d T(28)}{d \theta}=\frac{[m(15)+m(16)] h(17)-m(18) h(18)-T(18) \frac{d M_{C}}{d \theta}}{M_{C}}
$$


where

$$
\frac{d M_{C}}{d \theta}=m(15)+m(16)-m(18) .
$$

Equation 30 is also the mass balance for the condenser.

The condensation temperature, $T(17)$, used in Equation 23, is determined by the pressure in the condenser as follows:

$$
T(17)=f(P(17)) \text {. }
$$

Subroutine TVSP in Appendix A evaluates this expression. ${ }^{C}$ ', for Equation 23, is determined from the absorber and is discussed in Section 2.3.5.

\subsubsection{The Evaporator}

The evaporator is modeled as a heat exchanger in which the chilled water flows inside the tubes and is cooled by the evaporation of a liquid refrigerant (water) sprayed on the outside of the tubes. The transport equation can be written as:

$$
Q_{E}=m_{c h}\left(T_{c h}{ }^{\prime}-T_{c h}\right)=(U A)_{E} \frac{T_{c h}{ }^{\prime}-T_{c h}}{2 n\left[\frac{T_{c h}{ }^{\prime}-T(20)}{T_{c h}-T(20)}\right]} \text {. }
$$

This equation is rearranged to produce $T(20)$ as the dependent variable in the program. 
For the evaporator, the UA is calculated as it has been for the other components using Equation 2. The inside heat transfer coefficient is calculated by the turbulent expression (Equation 3) presented earlier. The outside heat transfer coefficient is determined either by

$$
h_{0}=0.8221\left(\frac{k^{3} g}{v^{2}}\right)^{1 / 3} \operatorname{Re}^{-0.22}
$$

if the outside film is laminar, or by

$$
h_{0}=0.0038 \operatorname{Re}^{0.4} \operatorname{Pr} 0.65\left(\frac{k^{3} g}{v^{2}}\right)^{1 / 3}
$$

if the flow is turbulent. The equation for the determination of laminar or turbulent flow is given by

$$
\mathrm{Re}_{\text {Transition }}=5,800 \mathrm{Pr}-1.06
$$

These equations are based on experimental data from Chun and Seban [13] for film flow on vertical tubes where the loading is defined as

$$
G=\frac{m}{L N} .
$$

For horizontal tubes, these correlations can be used if $G$ is given by

$$
G=\frac{m}{L(N)^{2 / 3}} .
$$


The film Reynolds number for these expressions (Equations 33 and 34 ) is calculated by

$$
\operatorname{Re}=\frac{4 G}{\mu}
$$

Because the evaporator has no mass storage, unlike the other major components of the system, the energy balance is simply

$$
Q_{E}=m(18)[h(20)-h(19)]
$$

and the mass balance is

$$
m(19)=m(20)
$$

The implication is that all refrigerant entering the evaporator is evaporated.

It should be pointed out that the evaporator is modeled somewhat fictitiously, because the refrigerant is always assumed to leave the evaporator in a saturated vapor condition. Since the evaporator chilled water exiting temperature is assumed to be fixed in the program and the chilled water flow rate is also fixed, the chilled water entering temperature must be the external parameter that changes. This method is unrealistic when considering actual machine operation, but it makes the reporting of machine performance easier, since most performance charts are reported at a fixed chilled water exiting temperature.

\subsubsection{The Absorber}

The absorber is modeled as a bundle of cooling tubes over which solution returning from the second generator is sprayed in the presence 
of refrigerant vapor from the evaporator. During this process, the refrigerant vapor is absorbed into solution. The combined heat of condensation and solution is then removed from the absorber through the cooling tubes. As in the condenser, the cooling tubes are situated above a storage trough.

For the absorber, the heat transfer is written as:

$$
Q_{A}=(U A)_{A} \frac{\left[T(7)-T_{C}{ }^{\prime}\right]-\left[T(8)-T_{C}\right]}{\frac{T(7)-T_{C}{ }^{\prime}}{T(8)-T_{C}}}
$$

Again, the UA is calculated by Equation 2. The inside heat transfer coefficient is given by the turbulent flow expression (Equation 25), and the outside heat transfer coefficient is assumed to be a constant with a value of $400 \mathrm{BTU} / \mathrm{hr} \mathrm{ft}^{2}{ }^{\circ} \mathrm{F}$. This value was selected after consultation with a representative of Arkla Industries, who indicated that heat transfer coefficients from 350 to $450 \mathrm{BTU} / \mathrm{hr} \mathrm{ft}{ }^{2}{ }^{\circ} \mathrm{F}$ can be obtained with proper solution distribution, tube spacing, and surface qualities in Arkla's absorption machines [14]. Furthermore, the solution leaving the tube bundle can be assumed to be at an equilibrium condition.

The decision to use a constant outside heat transfer coefficient and assume the equilibrium condition for the solution at the tube bundle exit was made because no suitable mathematical model could be defined. An extensive literature search, consultation with industry representatives [14], [15], and considerable independent work revealed that the coupled mass and heat transfer phenomenon occurring in the absorber was too physically and mathematically complex to be analyzed within the 
scope of this study. Thus, more reliable experimental data were used. Work is now being done, however, at The University of Texas at Austin to solve this problem for future absorber models.

$T(7)$ and $T(8)$, which are used in the heat transfer equation (Equation 41), are each assumed to be at an equilibrium condition and are evaluated at each state point's concentration and pressure as. follows:

$$
\begin{aligned}
& T(7)=f[P(7), X(7)] \\
& T(8)=f[P(8), X(8)]
\end{aligned}
$$

These functions are evaluated in the program by subroutine TEIPSOL. $T_{C}{ }^{\prime}$ is determined by the equation

$$
T_{C}^{\prime}=Q_{A} / m_{C}+T_{C}
$$

Two energy balance equations can be written for the absorber as a result of a separation of the cooling tubes and trough storage, as mentioned above. The energy equation for the tube section of the absorber is

$$
Q_{A}=m(20) h(20)+m(7) h(7)-m(8) h(8) .
$$

For the trough section, the energy equation is

$$
\frac{d}{d \theta}\left[M_{A} C_{p} T(10)\right]=m(8) h(8)-m(10) h(10)
$$


which, when simplified to the time derivative of $T(10)$, becomes

$\frac{d T(10)}{d \theta}=\frac{m(8) h(8)-m(10) h(10)-T(10) C_{p} \frac{d M_{A}}{d \theta}+0.7157 T(10) M_{A} \frac{d X(10)}{d \theta}}{M_{A} C_{p}}$.

The overall mass balance for the absorber is written as

$$
\frac{d M_{A}}{d \theta}=m(8)-m(10)
$$

which is also a term in the above energy equation. The species mass balance equation is

$$
\frac{d}{d \theta}\left[M_{A} X(10)\right]=m(8) \times(8)-m(10) \times(10)
$$

or in the form used in the program

$$
\frac{d x(10)}{d \theta}=\frac{m(8) \times(8)-m(10) \times(10)-x(10) \frac{d M_{A}}{d \theta}}{M_{A}}
$$

Additionally, a pressure drop is allowed for the refrigerant vapor which flows from the evaporator to the absorber. This is determined in the model by

$$
P(8)=P(20)-c[m(20)]^{2}
$$

The actual value of this pressure drop is small, but it has a considerable impact on system performance. The quantity $c$ is a coefficient whose influence is examined in Chapter 3. 


\subsubsection{The Heat Exchangers}

Both liquid-to-liquid heat exchangers are assumed to be counter flow-type shell and tube heat exchangers. In order to avoid specifying considerable geometry, a scaling approach was used to calculate the performance of each heat exchanger. A heat transfer coefficient was first calculated by hand for the tube side of the heat exchangers using Equation 25. It was then assumed that a geometry could be found so that the shell side heat transfer coefficient would be equal to the tube side. This was done for a "nominal condition," which is discussed further in Section 3.2. Then the shell side heat transfer coefficient was calculated as:

$$
h_{0}=h_{\text {nom }}\left(\frac{m}{m_{n o m}}\right)^{0.55}
$$

and the tube side as

$$
h_{i}=h_{\text {nom }}\left(\frac{m}{m_{n o m}}\right)^{0.8}
$$

The exponents in the above equations typify the effect of mass flow on heat transfer coefficients for the shell and tube sides respectively. The coefficients were combined using Equation 2. Then using the effectiveness-NTU method, the effectiveness of each counter flow heat exchanger was found by [16]

$$
\varepsilon=\frac{1-\exp [-N T U(1-C)]}{1-C \cdot \exp [-\operatorname{NTU}(1-C)]}
$$

where 


$$
N T U=\frac{U A}{C_{\min }}
$$

and

$$
c=\frac{C_{\min }}{C_{\max }}
$$

The quantities $C_{\min }$ and $C_{\max }$ are the minimum and maximum flow thermal capacitances, $\mathrm{mC}_{\mathrm{p}}$, for the two fluid streams.

\subsection{Model Limitations}

The computer model is a dynamic program that is capable of responding accurately to changing external conditions. For short periods after discontinuous inputs, errors may result.

Iterative variables are considered converged when

$$
\left|\frac{x_{\text {old }}-x_{\text {new }}}{x_{\text {old }}+x_{\text {new }}}\right|<0.0005
$$

This degree of accuracy has been found to be a reasonable compromise of accuracy and computer run time.

The time integration is broken into $0.005-\mathrm{hr}$ steps. This time interval has provided good results during test runs, but is only of importance when "transient solutions" are of interest: The size of the interval does not affect final steady state values.

A minor limitation is the modeling of the heat transfer in the first generator where the entering solution temperature in the log-mean 
temperature difference is assumed to be saturated. (The actual solution temperature is used in the overall heat balance; thus, energy is properly conserved.) This represents an error of only about $0.3 \%$ for the logmean temperature difference and gives a conservative value of heat transfer.

Similarly, the second generator assumes the incoming vapor to be saturated rather than slightly superheated in the log-mean temperature difference calculation. Although on the conservative side, this procedure results in no more than a $4 \%$ error at most conditions. (The second generator energy balance is modeled exactly.)

Frictional pressure drop is not used in the program, except that for the refrigerant between the evaporator and the absorber. With the exception of this pressure loss, most frictional losses are insignificant compared to the system pressure reduction between state point pairs 2-3, 5-7, 14-15, and 18-19. Careful system design could eliminate the remaining areas where frictional pressure drop could be of concern.

The model uses mass rather than volume for controlling solution storage. The reason was more for convenience than for accuracy of modeling. Relative changes in specific volume (or density) for typical operating ranges are shown in Table 2.1. Density changes are significant for components containing water-lithium bromide. The modeling for the first and second generators is the most critical since they maintain constant levels in practice but are assumed to maintain constant mass in the computer program. The absorber, on the other hand, is not as critical since it is free to adjust its level as the conditions warrant. 
Lastly, it should be pointed out that the boiling heat transfer data of Minchenko and Firsova [10] are being extrapolated to considerably lower heat flux levels than those for which the data were obtained. The accuracy of the results for the two generators is quite dependent upon these extrapolations. 
Table 2.1 VARIATION IN FLUID SPECIFIC VOLUME OVER EXTREME OPERATING RANGE

\begin{tabular}{llc}
\hline & & \multicolumn{1}{c}{$\begin{array}{c}\text { Percentage } \\
\text { Change in } \\
\text { Compecific Volume }\end{array}$} \\
\hline Evaporator & $40-90^{\circ} \mathrm{F}$ & 0.5 \\
Condenser & $75-175^{\circ} \mathrm{F}$ & 1.0 \\
First generator & $\begin{array}{l}0.5-0.6 \mathrm{C} \text { concentration } \\
200-300^{\circ} \mathrm{F}\end{array}$ & 3.0 \\
Second generator & $0.53-0.65$ concentration \\
& $\begin{array}{l}130-200^{\circ} \mathrm{F} \\
0.45-0.57 \text { concentration } \\
77-97^{\circ} \mathrm{F}\end{array}$ & 9.0 \\
Absorber & & 12.0 \\
\hline
\end{tabular}




\subsection{RESULTS}

\subsection{Method of Analysis}

Because the number of design and input parameters which may be varied in a water-7ithium bromide double-effect absorption unit is large, it is not feasible to develop performance data for the entire range of these quantities. For this reason the technique of varying one parameter at a time while holding all others at a "nominal condition" was applied. The result is a sensitivity scan of system performance for each of the variables being analyzed, compared to the nominal condition. To good approximation, the individual sensitivities can also be linearly combined over small variations to determine system performance when varying multiple parameters.

In this study, sensitivity scans were produced for the external variables of the system (such as cooling and chilled and source water temperatures) as well as design variables (such as heat exchanger areas, flow rates, and certain other internal flow control features). Table 3.1 provides a summary of the sensitivity scans performed. The second column represents the "nominal condition" about which individual variables were varied, and the third column indicates the range of variation for each. The table also identifies the figure which illustrates the effect on COP and capacity for each variable. Most of the performance sensitivity results presented herein are the work of Lawson [17].

\subsection{The Nominal Condition}

For this parametric analysis, a nominal condition for system operation was established. Appendix $D$ outlines the external constraints 
Table 3.1 NOMINAL CONDITIONS AND PARAMETER RANGES

\begin{tabular}{|c|c|c|c|c|}
\hline & Investigated Condition & Nominal Condition* & Range & Figure \\
\hline$T_{s}$ & Source hot water temperature & $280^{\circ} \mathrm{F}$ & $220-320^{\circ} \mathrm{F}$ & $3.2 \mathrm{a}$ \\
\hline $\mathrm{T}_{\mathrm{c}}$ & Cooling water temperature & $85^{\circ} \mathrm{F}$ & $50-i 00^{\circ} \mathrm{F}$ & $3.2 \mathrm{~b}$ \\
\hline$T_{c h}$ & Chilled water temperature & $44^{\circ} \mathrm{F}$ & $34-74^{\circ} \mathrm{F}$ & $3.2 \mathrm{c}$ \\
\hline$m_{s}$ & Source hot water flow rate & $5,000 \mathrm{lb} / \mathrm{hr}$ & $2,000-10,000 \mathrm{lb} / \mathrm{hr}$ & $3.3 a$ \\
\hline$m_{c}$ & Cooling water flow rate & $18,000 \mathrm{ib} / \mathrm{hr}$ & $8,000-28,000 \mathrm{lb} / \mathrm{hr}$ & $3.3 \mathrm{~b}$ \\
\hline $\mathrm{n}_{\mathrm{ch}}$ & Chilled water flow rate & $10,000 \mathrm{lb} / \mathrm{hr}$ & $5,000-15,000 \mathrm{lb} / \mathrm{hr}$ & $3.3 c$ \\
\hline $\mathrm{m}(10)$ & Solution flow rate & $900 \mathrm{lb} / \mathrm{hr}$ & $500-1,500 \mathrm{lb} / \mathrm{hr}$ & 3.4 \\
\hline$A_{G 1}$ & Area of first generator & $35 \mathrm{ft}^{2}$ & $5-105 \mathrm{ft}^{2}$ & $3.5 \mathrm{a}$ \\
\hline$A_{G 2}$ & Area of second generator & $20 \mathrm{ft}^{2}$ & $5-105 \mathrm{ft}^{2}$ & $3.5 \mathrm{~b}$ \\
\hline$A_{C}$ & Area of condenser & $20 \mathrm{ft}^{2}$ & $5-105 \mathrm{ft}^{2}$ & $3.5 c$ \\
\hline$A_{E}$ & Area of evaporator & $55 \mathrm{ft}^{2}$ & $5-105 \mathrm{ft}^{2}$ & $3.5 d$ \\
\hline$A_{A}$ & Area of absorber & $60 \mathrm{ft}^{2}$ & $5-105 \mathrm{ft}^{2}$ & $3.5 \mathrm{e}$ \\
\hline$A_{H \times 1}$ & Area of first heat exchanger & $5 \mathrm{ft}^{2}$ & $0-20 \mathrm{ft}^{2}$ & $3.5 f$ \\
\hline$A_{H X 2}$ & Area of second heat exchanger & $5 \mathrm{ft}^{2}$ & $0-20 \mathrm{ft}^{2}$ & 3.59 \\
\hline \multirow[t]{6}{*}{ c } & $\begin{array}{l}\text { Pressure drop coefficient between } \\
\text { the evaporator and the absorber }\end{array}$ & $0.5 \times 10^{-6}$ & $0-5 \times 10^{-6}$ & 3.6 \\
\hline & Orifice flow control (varying $T_{s}$ ) & & $220-320^{\circ} \mathrm{F}$ & 3.7 \\
\hline & Centrifugal pump I (varying $T_{s}$ ) & & $220-320^{\circ} \mathrm{F}$ & $3.8 \mathrm{a}$ \\
\hline & Centrifugal pump 11 (varying $T_{s}$ ) & & $220-320^{\circ} \mathrm{F}$ & $3.3 \mathrm{~b}$ \\
\hline & $\begin{array}{l}\text { Orifice flow control with } \\
\text { centrifugal pump I (varying } T_{s} \text { ) }\end{array}$ & & $220-320^{\circ} \mathrm{F}$ & $3.10 \mathrm{a}$ \\
\hline & $\begin{array}{l}\text { Orifice flow control with } \\
\text { centrifugal purlp II (varying } T_{s} \text { ) }\end{array}$ & & $220-320^{\circ} \mathrm{F}$ & $3.10 \mathrm{~b}$ \\
\hline
\end{tabular}

*The nominal condition corresponds to a cooling capacity of approximately 10 tons. 
chosen for this "Nominal Condition." An attempt was made to apply the same external parameters used by industry wherever applicable, but others were selected to provide a reasonable range for the analys is of a 10-ton unit.

External conditions, including cooling water temperature, cooling water flow rate (per ton), and chilled water exiting temperature are standard values for the Trane "Two-Stage Absorption Cold Generator" units at their design conditions [18]. Inlet hot water temperature, inlet hot water flow rate, and chilled water flow rate were arbitrarily picked near the middle of a reasonable range for 10 -ton units.

The nominal condition also requires that certain geometrical constraints be defined. Al1 the tubes in the various heat exchange components were chosen to be $1 / 2$ inch in outside diameter. The number of tubes in each unit was selected to obtain velocities of approximately $4 \mathrm{ft} / \mathrm{sec}$. This velocity allowed reasonably good heat transfer characteristics but avoided large pressure drops.

The area ratio $(A R)$, defined as the ratio of the outside to inside heat transfer area, was chosen arbitrarily to be 1.08 . This ratio was considered reasonable and consistent with currently available heat exchanger tubing.

The areas of the heat exchange components chosen were based on two constraints. The first was that the total capacity at the nominal condition be 10 tons. The second required that changes in the areas of each of the components must have an approximately equal effect on the capacity of the system. 
Additionally, certain flow control methods were specified for the nominal condition. The solution flow rate was fixed, assuming the use of a constant displacement pump. The refrigerant flow through the second generator was assumed to be controlled by a float-type valve, which required all the refrigerant to be in the condensed phase (1iquid) at the generator exit.

Finally, the nominal condition was assumed to have a pressure drop between the evaporator and the absorber of $5 \%$ of the absolute pressure in the evaporator. This corresponds to a coefficient of $5 \times 10^{-7}$ [see Equation (51)].

Figure 3.1 a illustrates the state points of a 10 -ton system characterized by the external constraints mentioned above and defines the nominal condition. Figure $3.1 \mathrm{~b}$ presents the state points for the double-effect cycle operating at the nominal condition on a pressuretemperature-concentration diagram. It is important to note that many computer runs were made to iterate toward this chosen nominal condition.

\subsection{Individual Sensitivity Scans}

The individual scans are tabulated in Table 3.1 in the order in which they are discussed below. The range for each variable is also specified in the table.

Figures 3.2 through 3.6 present individual performance curves for each external and design variable of the system, with all other variables remaining at the nominal condition. Figures 3.7 through 3.10 illustrate the effect on performance of changing various control methods in the system. For example, most double-effect systems would use a 


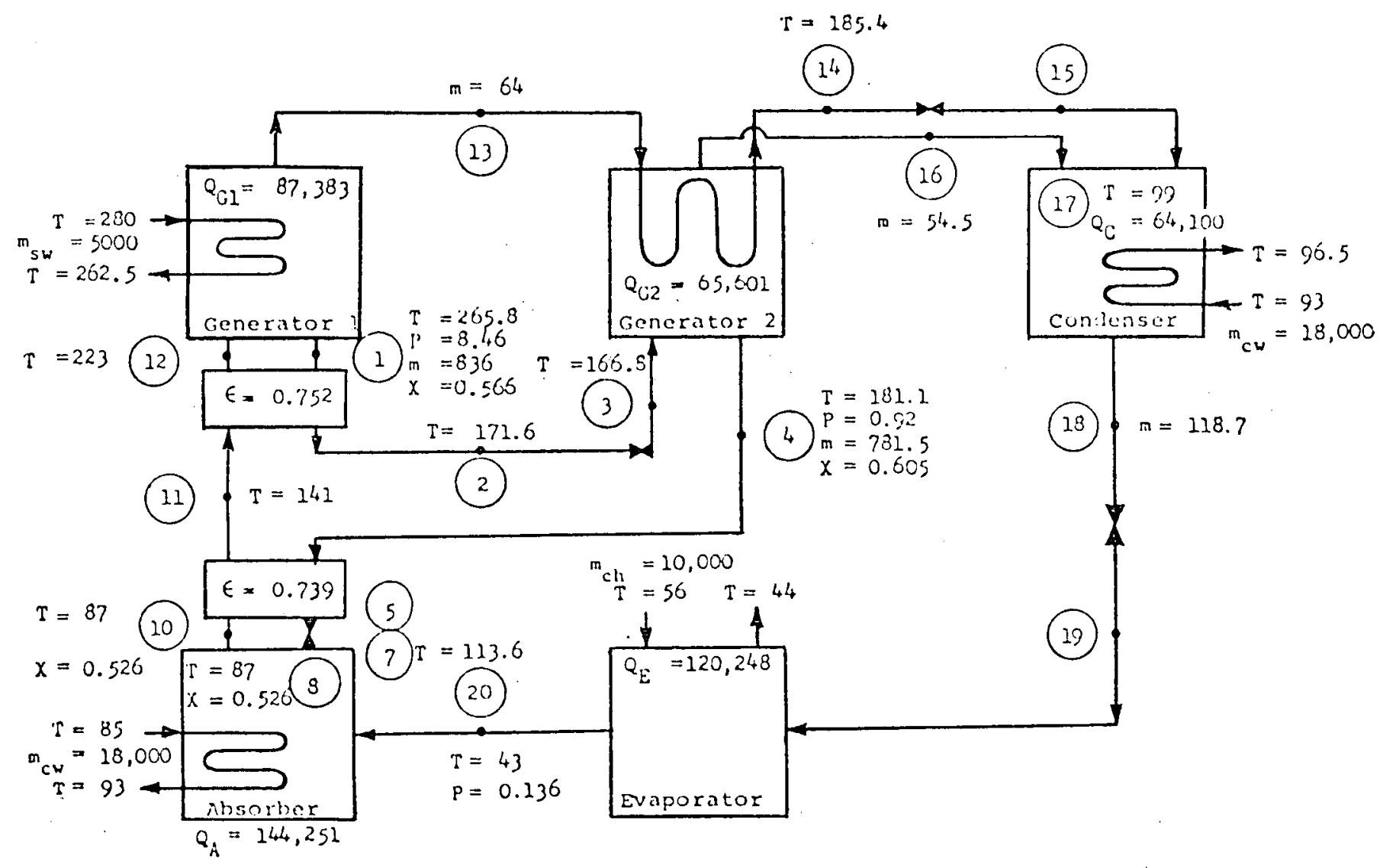

Figure 3.1a THE NOMINAL CONDITION 


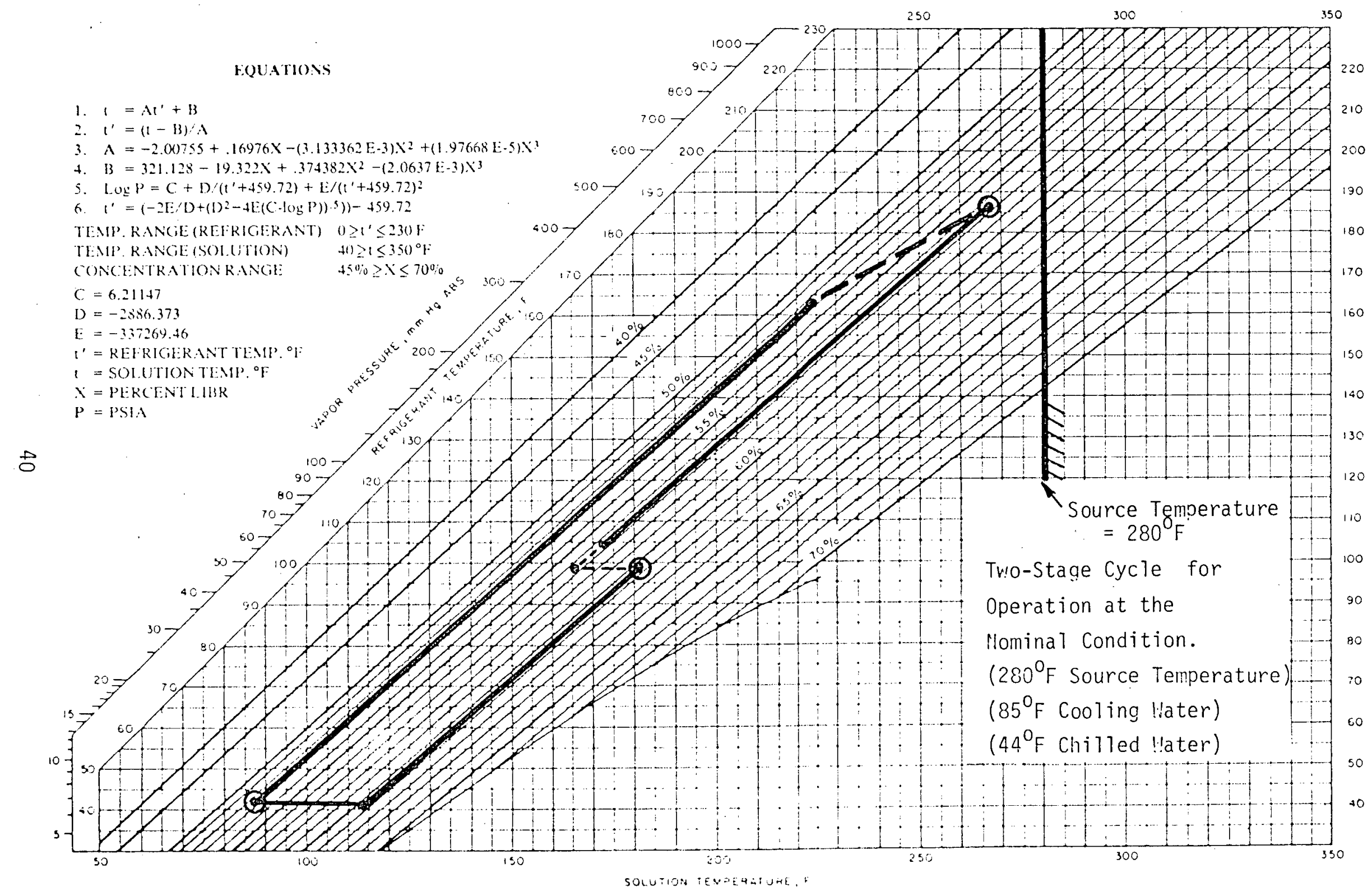

Figure 3.1b TWO-STAGE WATER-LITHIUM BROMIDE CYCLE FOR OPERATION AT THE NOMINAL CONDITION 
centrifugal solution pump rather than the constant displacement pump specified by the nominal condition. Thus, to study this effect, two pumps with different head-flow characteristics are examined. Additionally, the more commonly used orifice flow control, rather than the float control method specified by the nominal condition, is investigated. Then, the combinations of orifice flow and centrifugal pump flow are explored.

For each scan, the fractional capacity and the coefficient of performance are plotted as functions of the changing variable. (The actual machine capacity can be determined by multiplying the fractional capacity by 10-tons. Note that at the nominar condition, the fractional capacity is 1.0 , or 10 tons.) The curve characteristics are also discussed and operational limitations of the variables are defined in the following paragraphs.

\subsubsection{Scans Based on the Nominal Condition}

(a) Source Hot Water Temperature: Figure 3.2a shows the effect on performance of the source hot water temperature. As the hot water temperature increases, the COP increases asymptotically to about 1.37. The capacity, however, increases in an approximately linear fashion. The source hot water temperature is limited on the high end by the crystalization limit, which occurs at $320^{\circ} \mathrm{F}$. It is limited at low temperature to about $190^{\circ} \mathrm{F}$, where the COP and capacity approach zero.

Another interesting trend is the shifting of the concentration levels in the system with an increase in operating temperature. The weakest concentration of the cycle (occurring in absorber) will change 


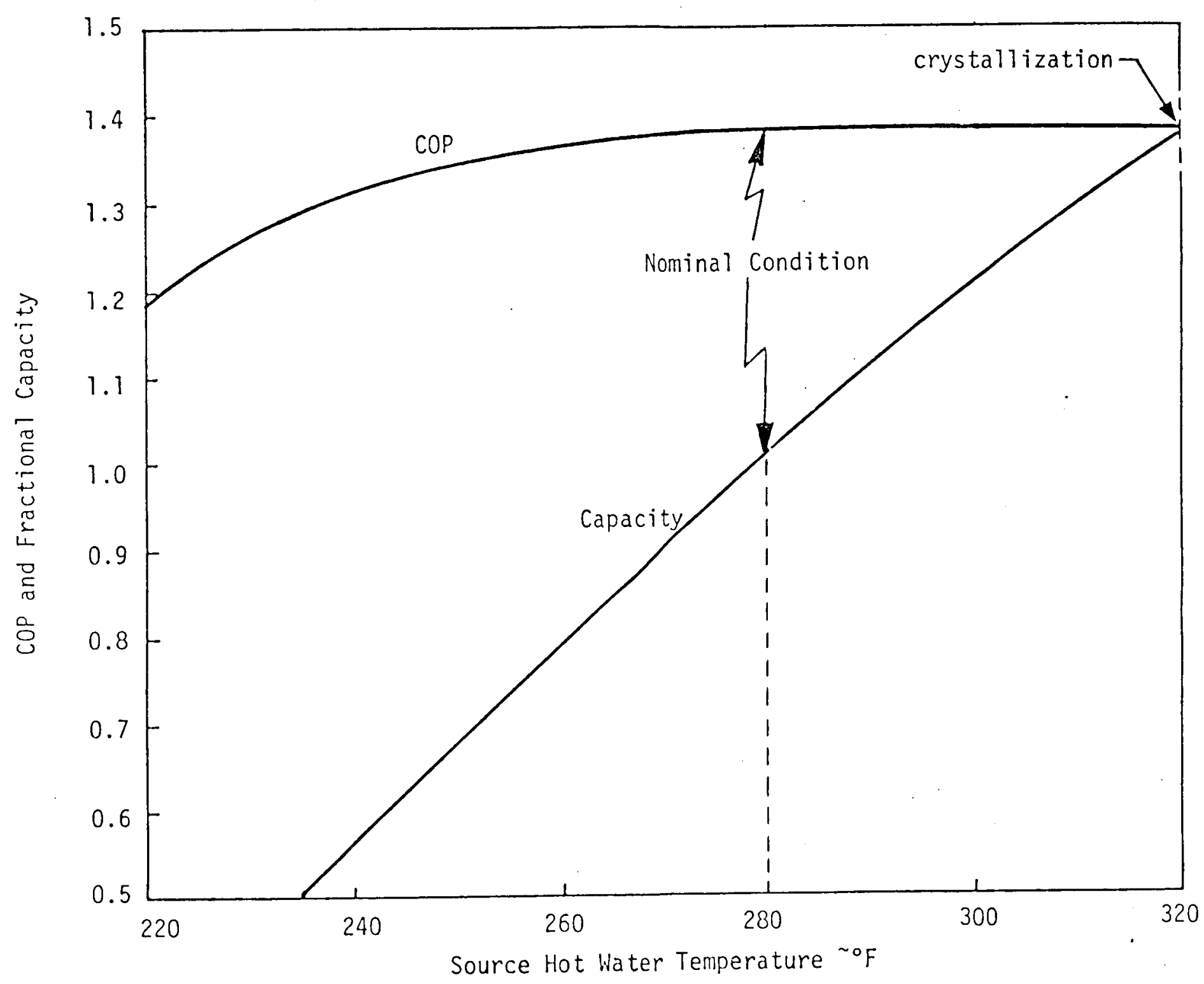

Figure 3.2a EFFECT OF SOURCE HOT WATER TEMPERATURE ON PERFORMANCE 
from $0.5161 \mathrm{~b} \mathrm{LiBr} / 1 \mathrm{~b}$ solution at $220^{\circ} \mathrm{F}$ to 0.533 at $320^{\circ} \mathrm{F}$. This difference is caused by the change in generator temperature and is allowed by an increase in the refrigerant storage in the condenser.

(b) Cooling Water Temperature: Figure 3.2b shows the effect of cooling water temperature. The COP remains nearly constant until rather high-temperature cooling water is used, beyond which system performance degrades sharply. The capacity of the system, on the other hand, is a nearly linear decreasing function of cooling water temperature. The cooling water temperature is limited at low temperature by the crystalization limit, which occurs near $52^{\circ} \mathrm{F}$. The upper range is limited to approximately $108^{\circ} \mathrm{F}$, where both COP and capacity approach zero.

(c) Chilled Water Temperature: Figure 3.2c shows the effect of the chilled water exiting temperature on system performance. As this temperature increases, the COP asymptotically increases to a maximum of about 1.54, and the capacity increases in a linear fashion. The lower limit on chilled water temperature is determined by the approach temperature in the evaporator and the freezing point of water. The upper limit is determined only by the application for which the chilled water is used. These results show that for highest machine performance, the highest chilled water temperature that is acceptable for the application should be used. However, for air-conditioning purposes, chilled water temperatures above $60^{\circ} \mathrm{F}$ are normally inadequate.

(d) Source Hot Water Flow Rate: The effect of source hot water flow rate is shown in Figure $3.3 \mathrm{a}$. The COP is, for all practical purposes, independent of this parameter. The capacity of the system, however, is dependent on source hot water flow rate at low flows, but asymptotically approaches 1.05 (10.5 tons) for high flows. 


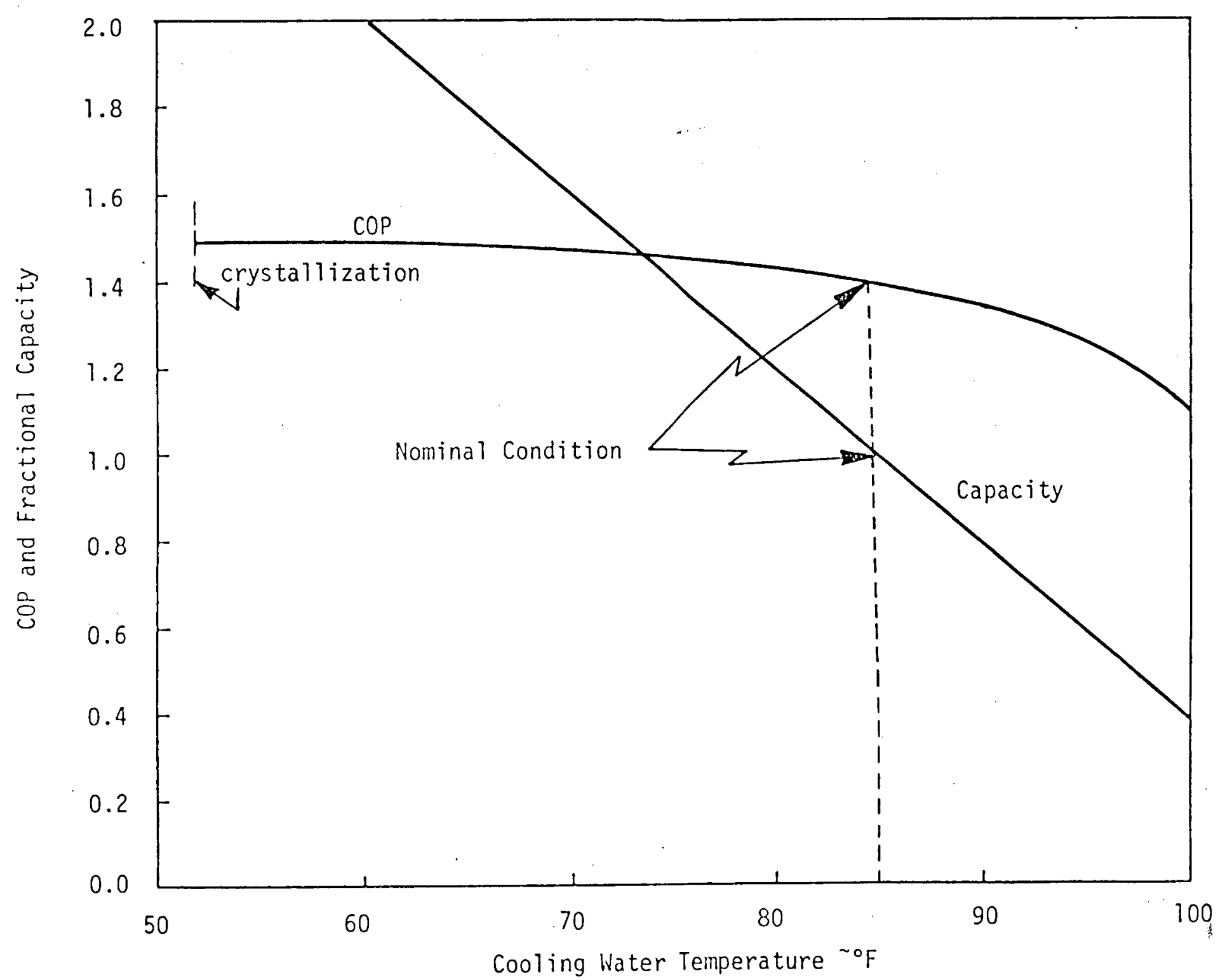

Figure 3.2b EFFECT OF COOLING WATER TEMPERATURE ON PERFORMANCE 


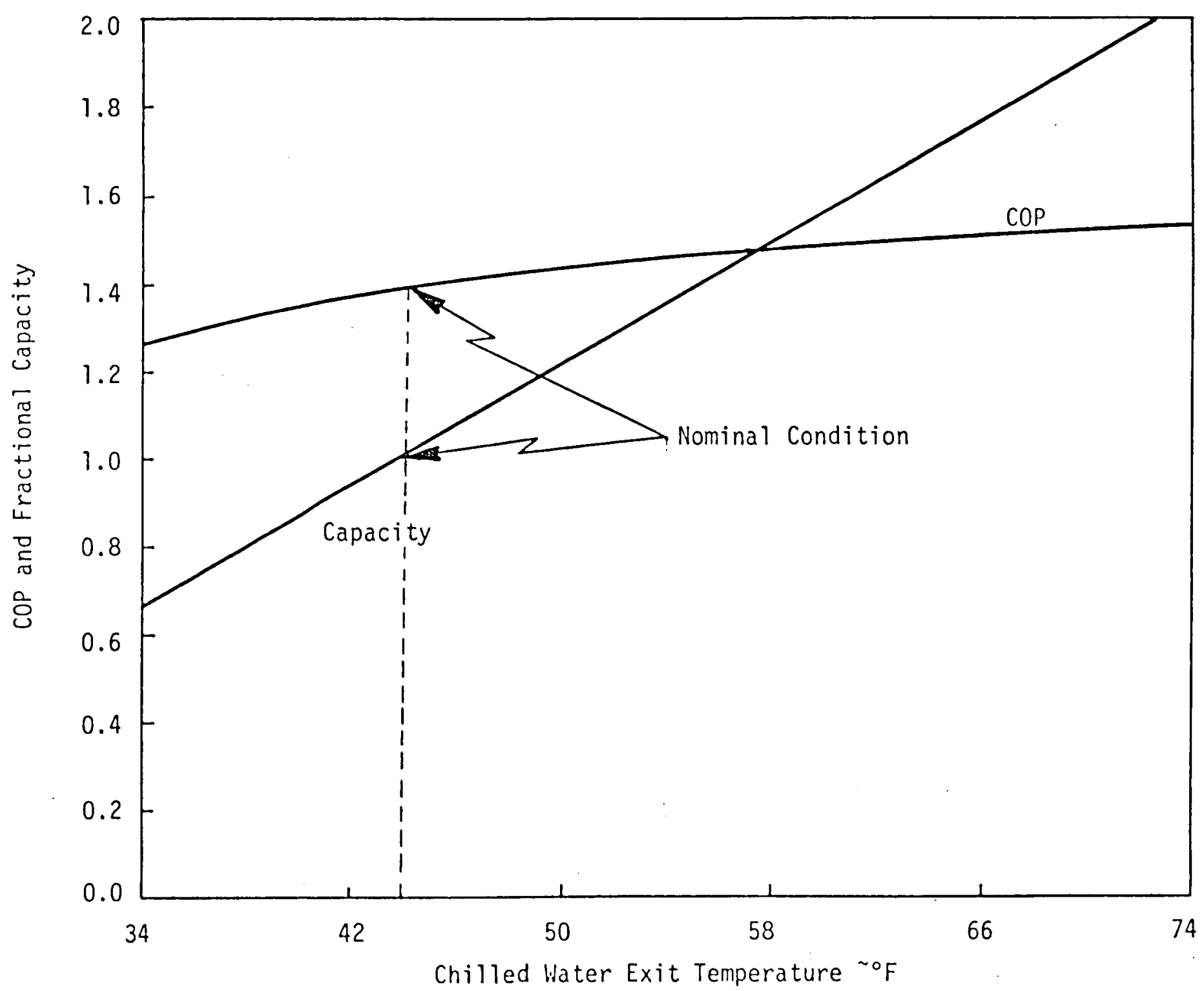

Figure $3.2 \mathrm{C}$ EFFECT OF CHILLED WATER EXIT TEMPERATURE ON PERFORMANCE 


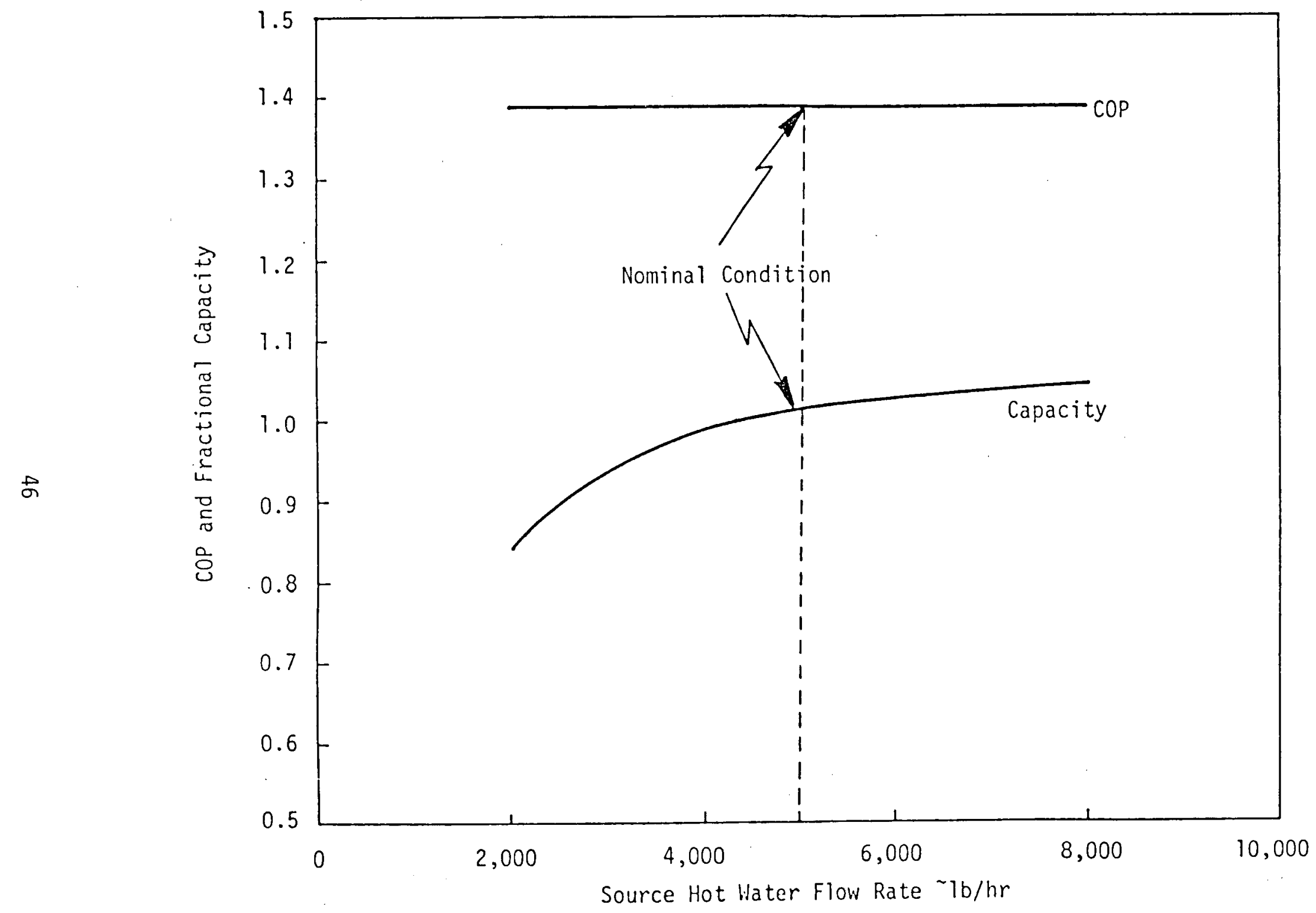

Figure 3.3a EFFECT OF SOURCE HOT WATER FLOW RATE ON PERFORMANCE 
(e) Cooling Water Flow Rate: Figure $3.3 \mathrm{~b}$ gives the system performance as a function of cooling water flow rate. Both COP and capacity are asymptotic functions of this variable. The COP reaches a limiting value of 1.4, while the capacity approaches about 1.2. This study examined the cooling water flowing only in series between the absorber and condenser. Future work should include a study of the effect of cooling water flow in reverse series and in parallel to determine the most efficient use of cooling water for these systems.

(f) Chilled Water Flow Rate: The chilled water flow rate as shown in Figure $3.3 \mathrm{c}$ does not significantly affect system performance. The very slight decrease in capacity with flow rate is due primarily to the method used in the program model, which specifies the chilled water exit temperature. As the flow rate increases, the chilled water inlet temperature decreases, resulting in a lower effective temperature difference for heat transfer. This lower effective temperature difference is, however, offset by a rising overall heat transfer coefficient due to increasing the flow. The evaporator was configured so that the outside heat transfer coefficient had the dominant effect on the overall heat transfer coefficient, but an evaporator could be designed in which the inside heat transfer coefficient is dominant and the chilled water flow rate would then have a more pronounced effect. This effect would still be small compared to those other system parameters.

(g) Solution Circulation Rate: Figure 3.4 shows the performance due to variations in solution circulation rate. With increased circulation, the COP decreases almost linearly. Increasing the flow rate increases the capacity asymptotically toward about 1.1. Increasing 


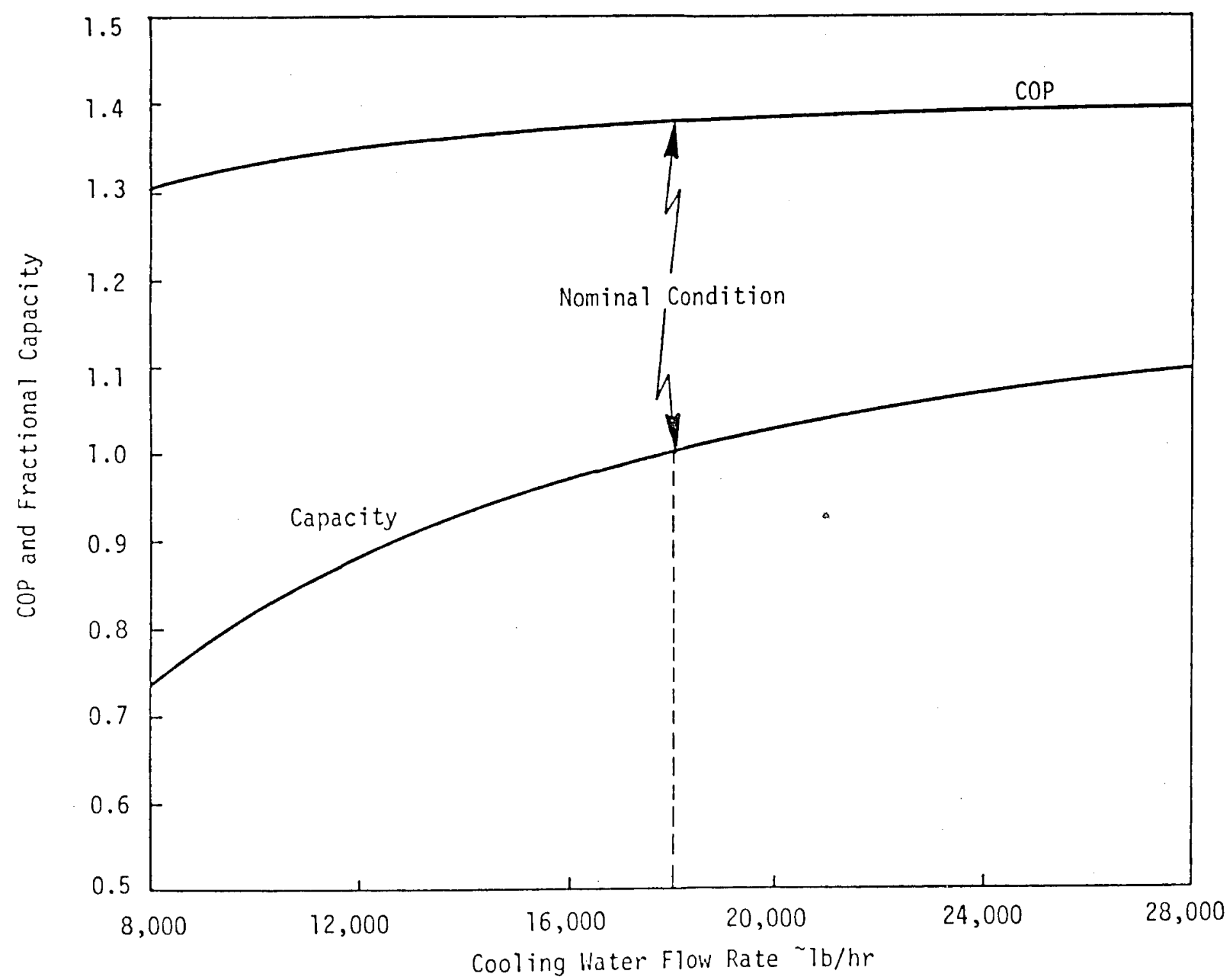

Figure 3.3b EFFECT OF COOLING WATER FLOW RATE ON PERFORMANCE 


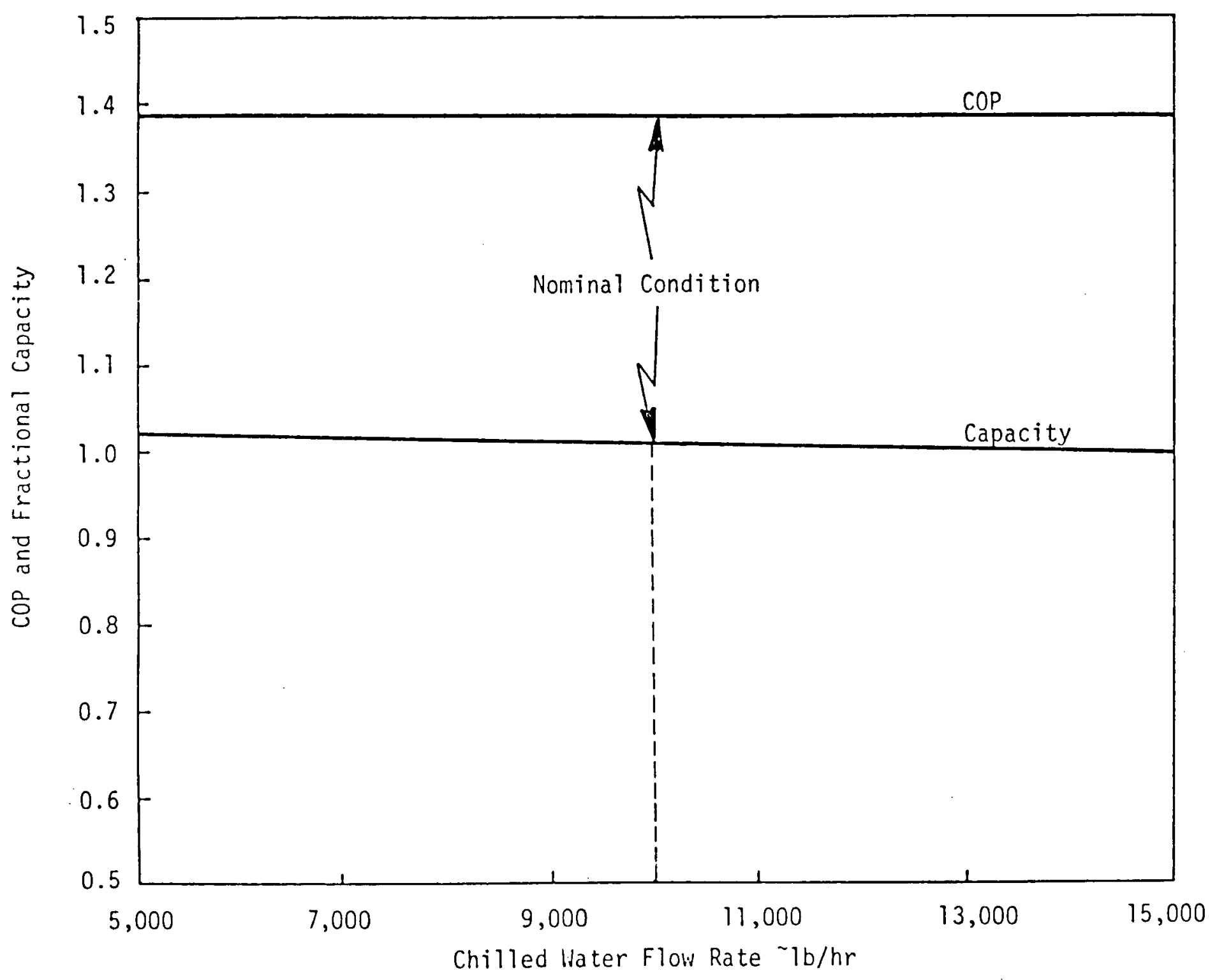

Figure 3.3C EFFECT OF CHILLED WATER FLOW RATE ON PERFORMANCE 


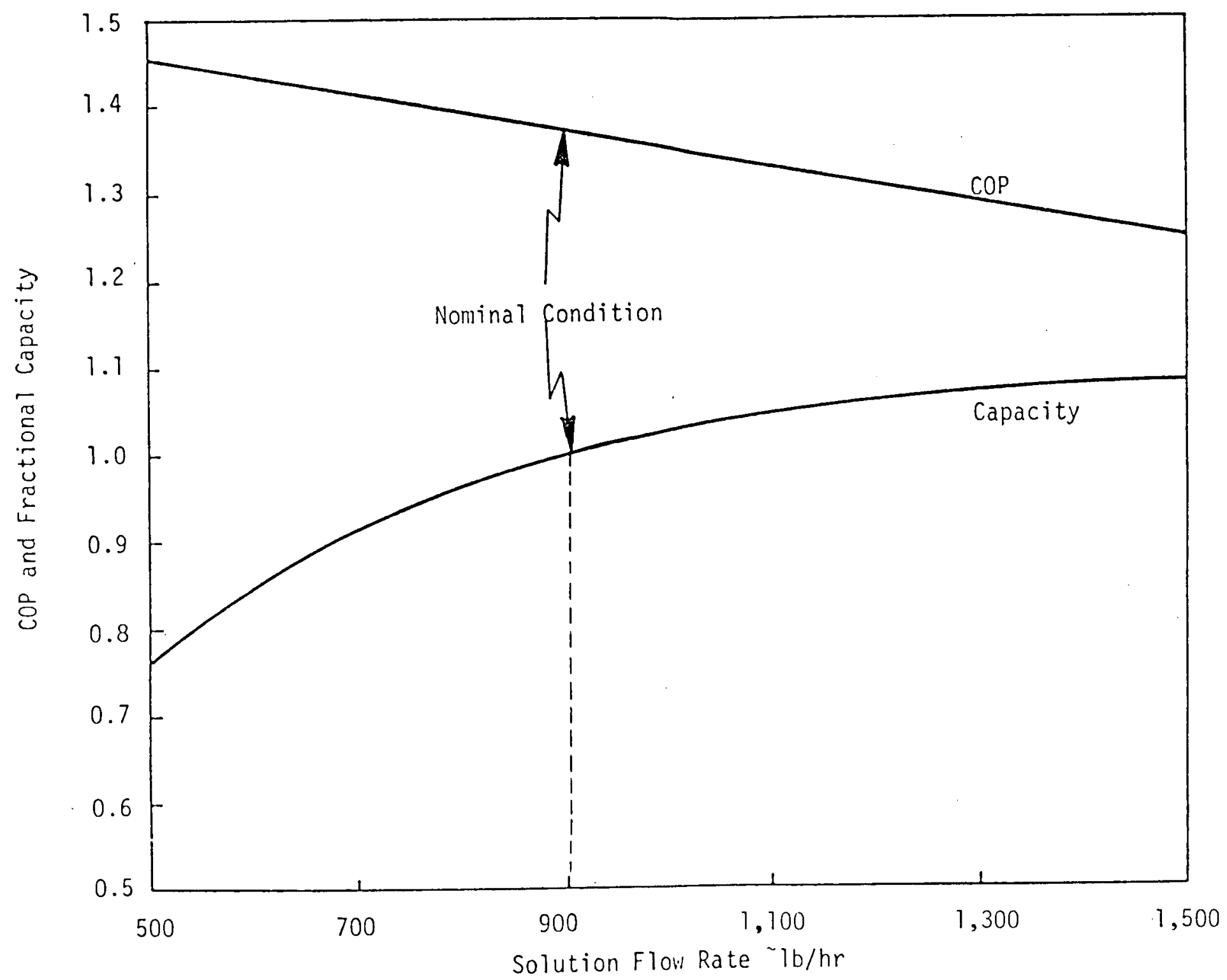

Figure 3.4 EFFECT OF SOLUTION FLOW RATE ON PERFORMANCE 
the circulation rate also has a tendency to narrow the concentration range over which the system operates. This affects the pressure levels in the system and makes the second generator more effective at lower circulation rates, a condition which in turn increases the COP, but at the expense of capacity. An increased circulation rate also allows greater heat transfer to occur in the generators, thus raising system capacity.

(h) Areas of Generators, Condenser, Evaporator, and Absorber: The next series of figures shows the effects on performance of varying the heat transfer surface areas of the different heat transfer components. The first, Figure 3.5a, shows the effect of changing the first generator's surface area. The nominal area for the generator is fairly high on the curve, indicating a larger generator area alone will not produce a significantly larger COP or capacity. This is also true of Figures 3.5b-e, which illustrate the effects of the second generator, condenser, evaporator, and absorber areas. The slopes at the nominal conditions are similar because of the selection criteria for the nominal design condition described in Section 3.2. This means that a small change in surface area in any of these components will produce essentially the same effect on system performance.

(i) Heat Exchanger Areas: While both COP and capacity are affected by the heat exchange components discussed above, the influence of the areas of the two heat exchangers on performance is quite different. The COPs are shown in Figures $3.5 \mathrm{f}$ and $3.5 \mathrm{~g}$ to be strong functions of the heat exchanger areas, while capacities are very weakly affected. Heat exchanger 1 affects capacity only slightly, and heat exchanger 2 


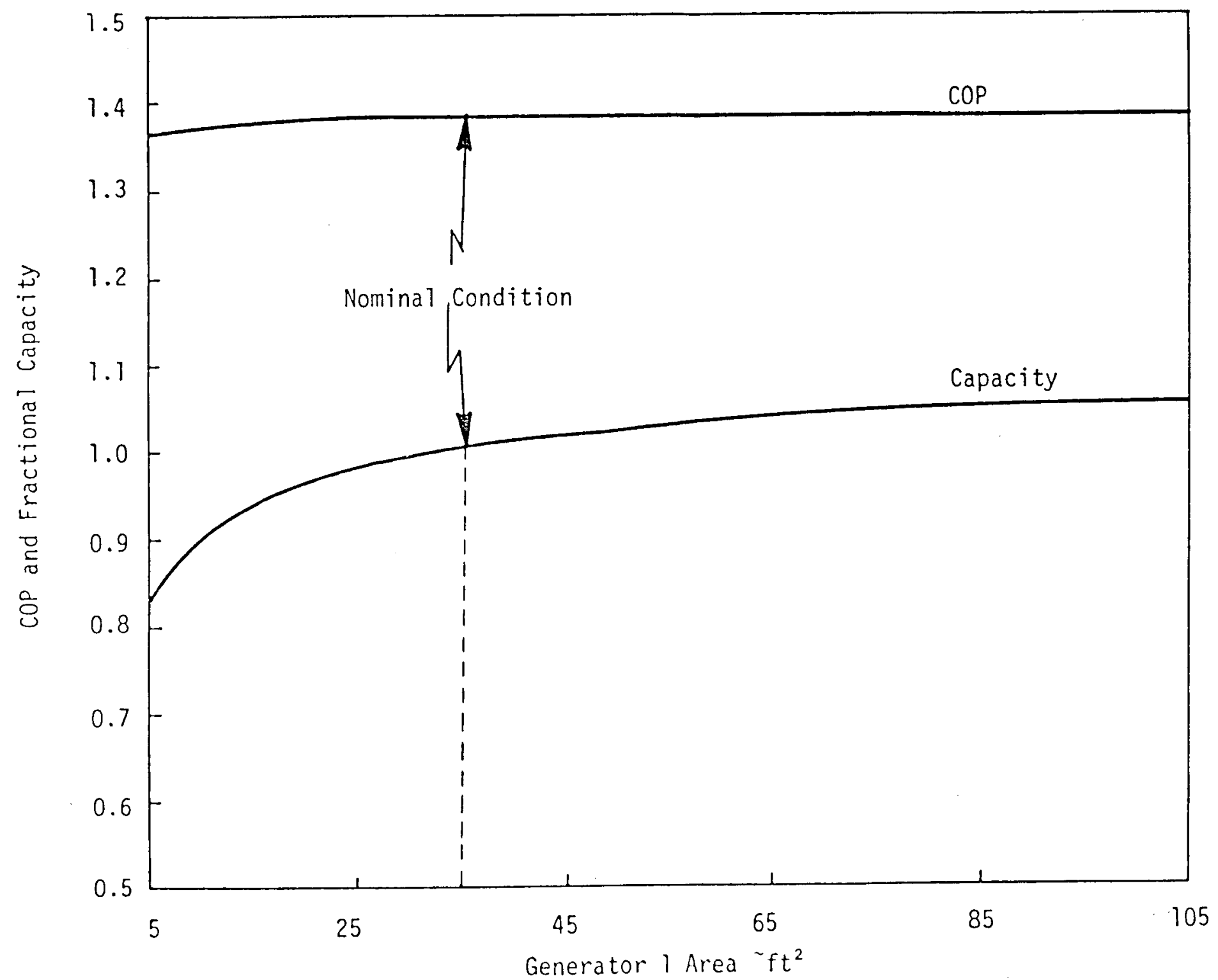

Figure $3.5 \mathrm{a}$ EFFECT OF GENERATOR I AREA ON PERFORMANCE 


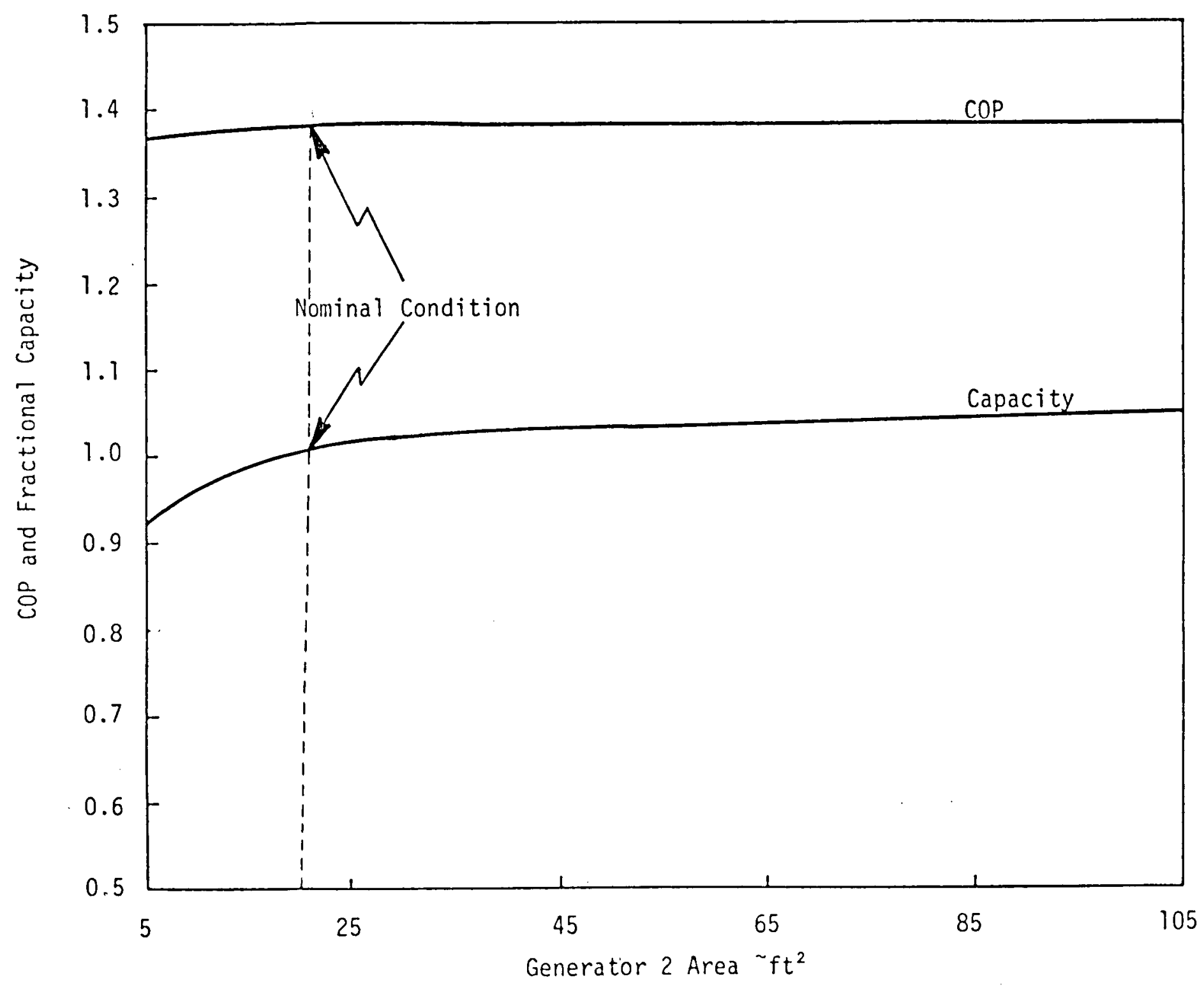

Figure $3.5 \mathrm{~b}$ EFFECT OF GENERATOR 2 AREA ON PERFORMANCE 


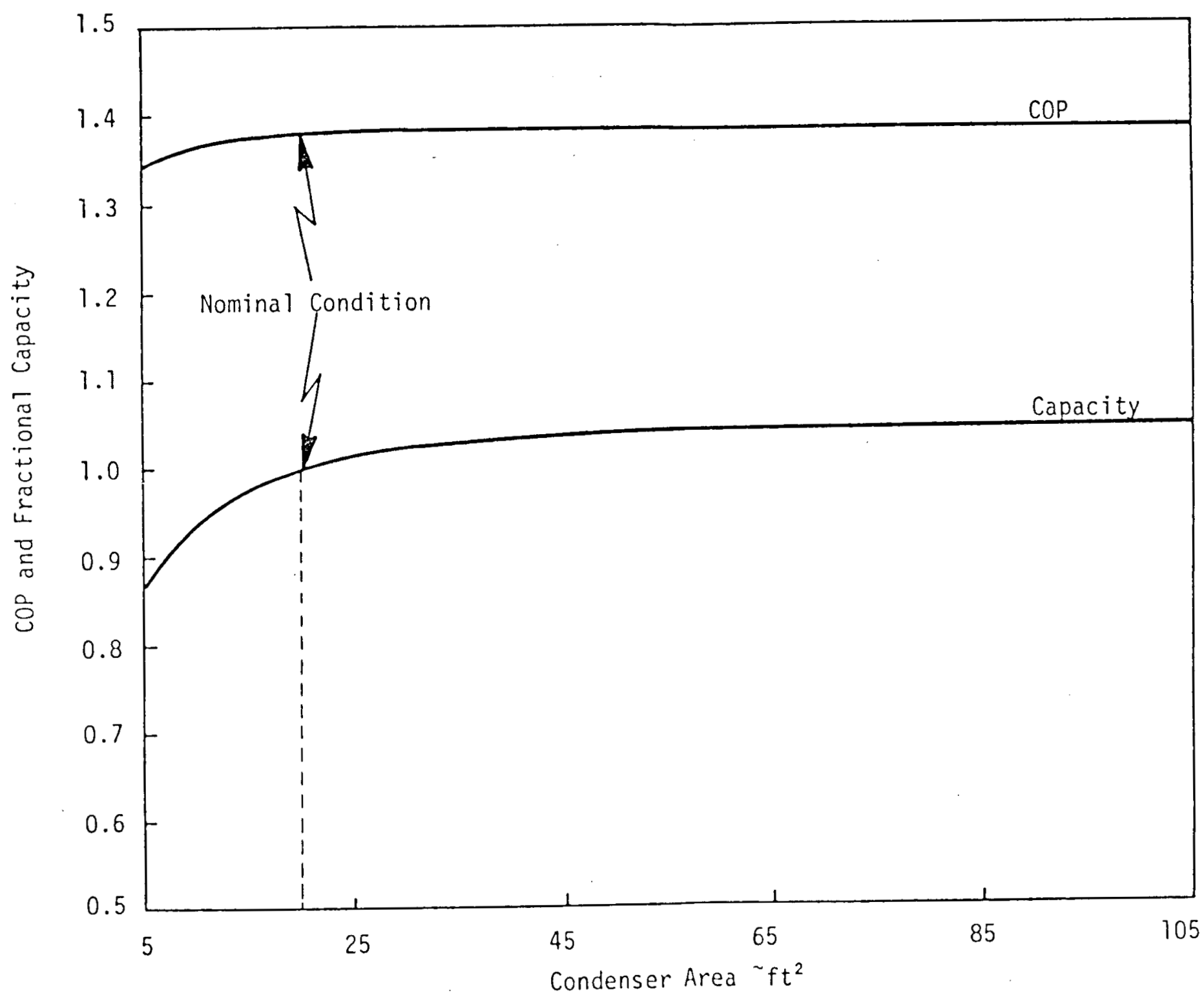

Figure $3.5 \mathrm{C}$ EFFECT OF CONDENSER AREA ON PERFORMANCE 


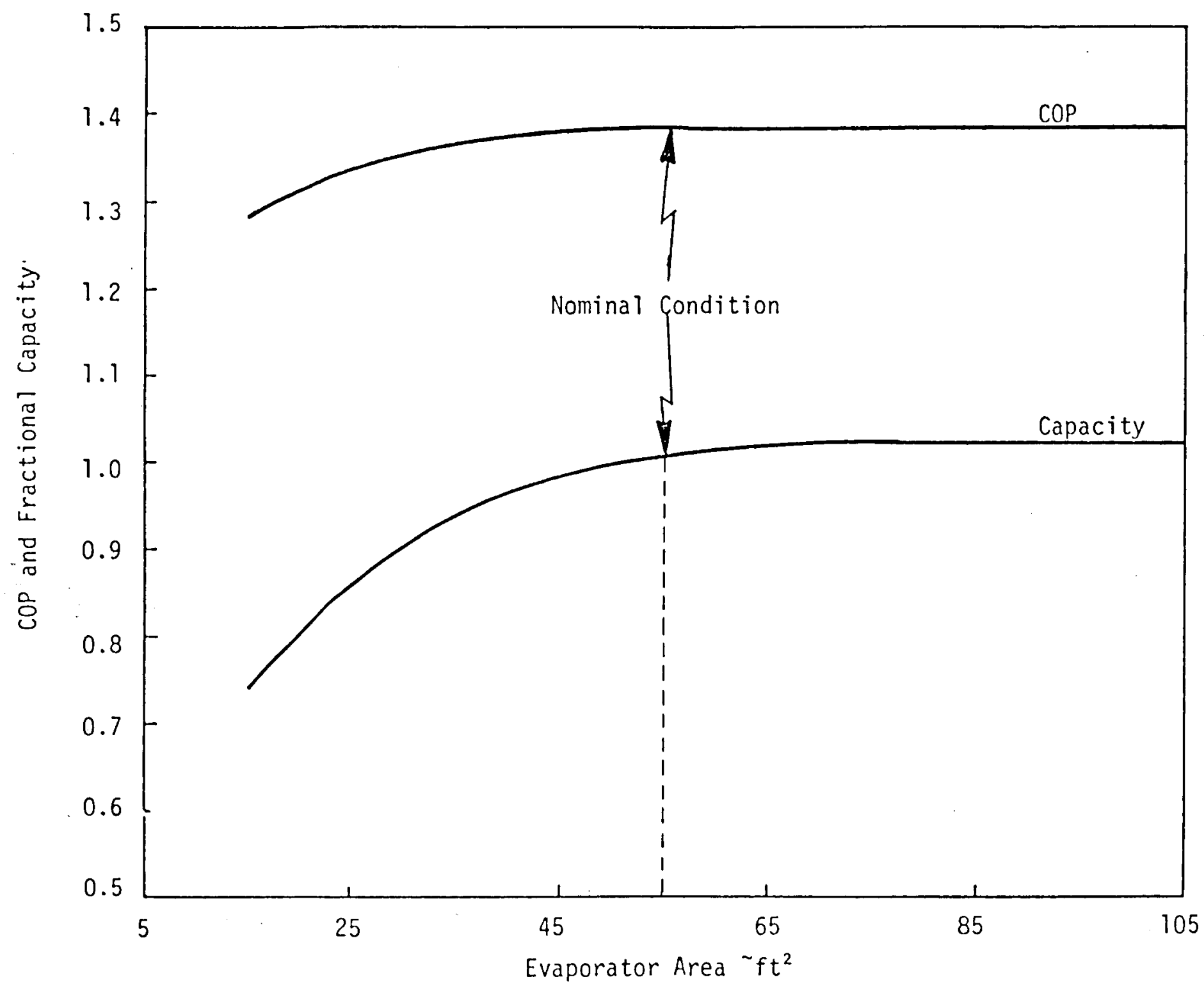

Figure $3.5 \mathrm{~d}$ EFFECT OF EVAPORATOR AREA ON PERFORMANCE 


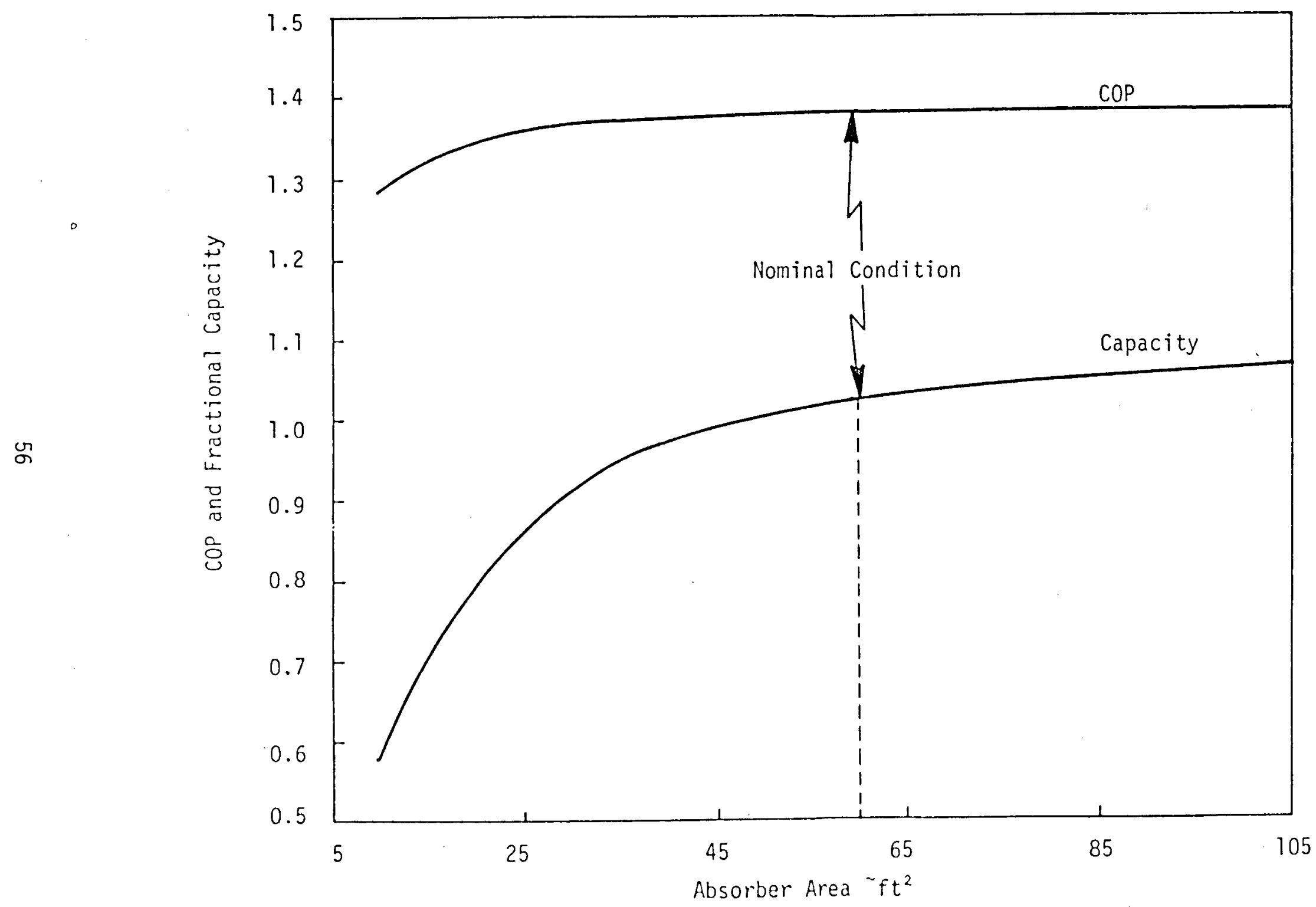

Figure 3.5e EFFECT OF ABSORBER AREA ON PERFORMANCE 


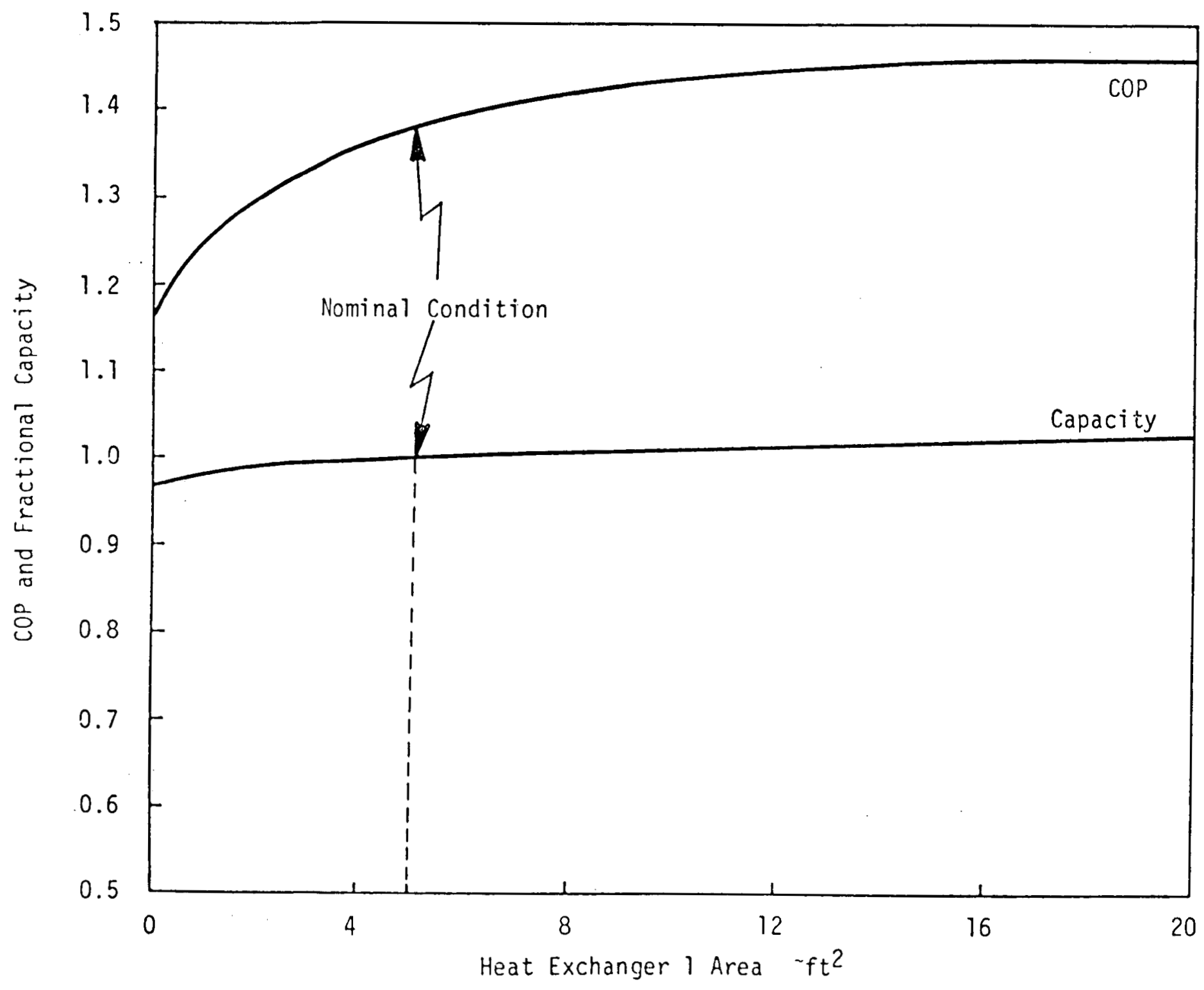

Figure $3.5 \mathrm{f}$ EFFECT OF HEAT EXCHANGER 1 AREA ON PERFORMANCE 


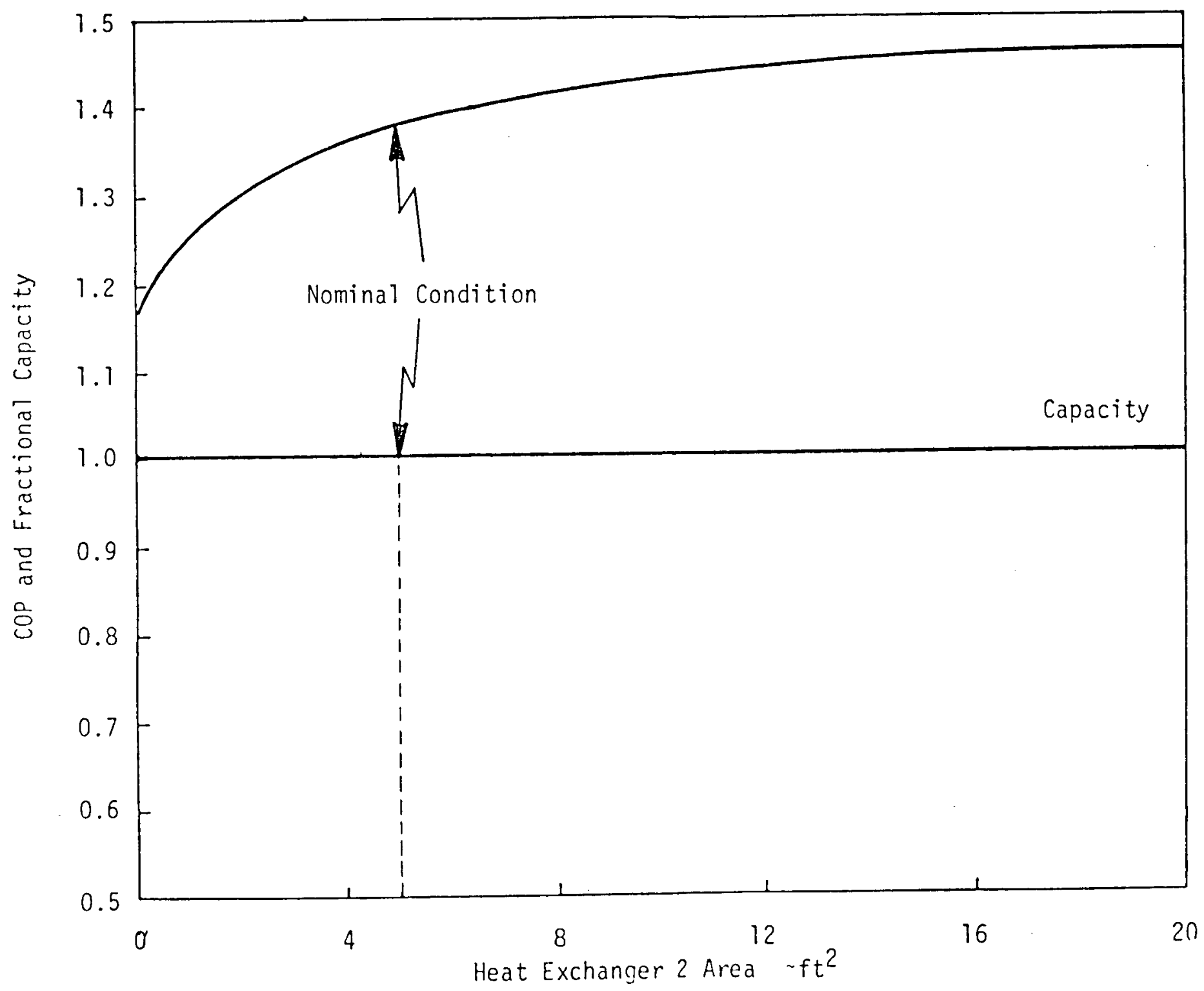

Figure $3.5 \mathrm{~g}$ EFFECT OF HEAT EXCHANGER 2 AREA ON PERFORMANCE 
has virtually no effect on capacity. These results indicate that the proper selection of heat exchanger areas should be a major consideration during system design, in order to yield optimum performance in terms of COP and capacity. These results also show that the two heat exchangers are almost equally important to the overall system performance.

Figure 3.6 shows the importance of minimizing the pressure drop between the evaporator and the absorber, particularly because of the effect of the pressure drop on capacity. Although it is not great in absolute terms, this pressure drop is important because of the extremely low pressures that exist in the two components. At the nominal condition the evaporator operates at 0.136 psi. With the nominal $5 \%$ pressure loss $\left(c=5 \times 10^{-7}\right)$, the absorption process takes place at $0.129 \mathrm{psi}$. This is a pressure reduction of only $0.007 \mathrm{psi}(0.4 \mathrm{~mm}$ of $\mathrm{Hg})$, yet it results in a reduction in capacity of approximately $5 \%$. Figure 3.6 also shows that a $50 \%$ pressure drop $\left(c=5 \times 10^{-6}\right)$ or $0.068 \mathrm{psi}(3.5 \mathrm{~mm}$ of $\mathrm{Hg})$ can reduce the COP by $6 \%$ and the capacity by $30 \%$. Thus, this parameter is of considerable importance in the design of absorption systems.

\subsubsection{Effect of Orifice Flow Control}

All of the above results are based on a float control device which requires that all refrigerant vapor from the first-effect generator be condensed in the second-effect generator. The following results examine the comparable performance when orifice control is used. The size of the orifice is optional, but in the results that follow the orifice was sized such that at the nominal condition $\left(280^{\circ} \mathrm{F}\right)$ all of the refrigerant vapor from the first effect is condensed in the second effect. That is, 


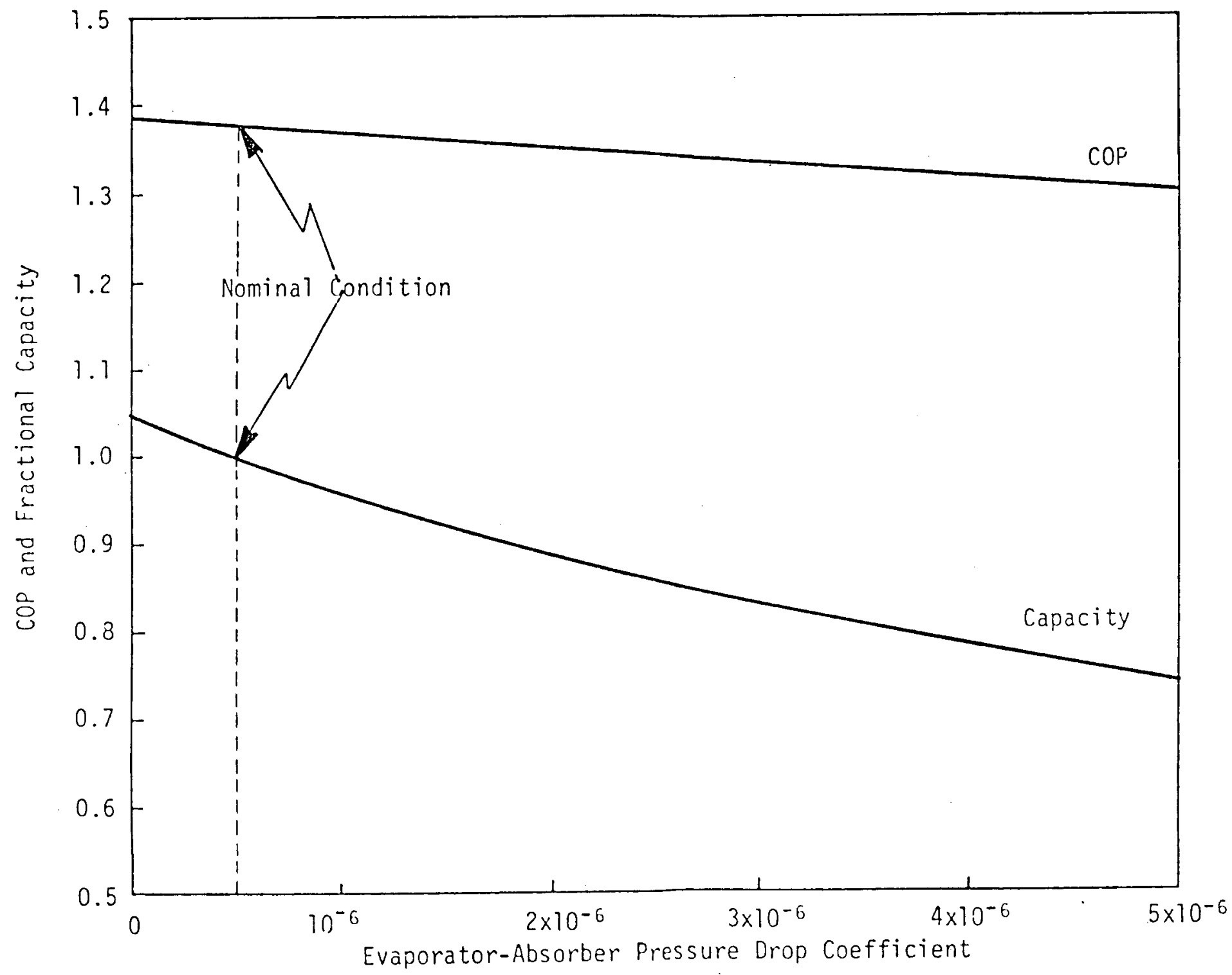

Figure 3.6 EFFECT OF EVAPORATOR-ABSORBER FLOW COEFFICIENT ON PERFORMANCE 
at the nominal conditions the two designs will give the same performance. At other than the nominal condition differences will arise because complete condensation of refrigerant may not occur with orifice control.

Figure 3.7 shows the effect of source hot water temperature on performance for the nominal system run with an orifice-type of flow control device between the second generator and the condenser. Comparing this to the float control results in Figure $3.2 \mathrm{a}$ shows that the COP curve for the orifice device is generally lower than the COP curve for the float device, but the two curves become almost tangent in the range of 275 to $310^{\circ} \mathrm{F}$ source hot water temperature. In fact, if a series of different-sized orifices were used, a family of COP curves would be generated, with the maximums from these curves effectively representing the performance locus for the float control device of Figure 3.2a.

When an orifice control device is used, the COP drops considerably faster at lower temperatures than with the float control device, because the refrigerant vapor flow to the second generator cannot be totally condensed. For example, at a source temperature of $225^{\circ} \mathrm{F}$, the quality of the refrigerant leaving the second generator is $99 \%$. The system is then effectively operating as a single-effect unit. At the other extreme, with a source temperature of $320^{\circ} \mathrm{F}$, the system could condense even more refrigerant in the second generator. The system under these conditions is capable of totally condensing the flow and partially subcooling it. This effect limits the performance at high temperatures and causes the COP curves to begin to slope down. It also decreases the slope of the capacity curve. For comparison, the performance with the float control device is presented in Figure 3.7 as dashed curves. 


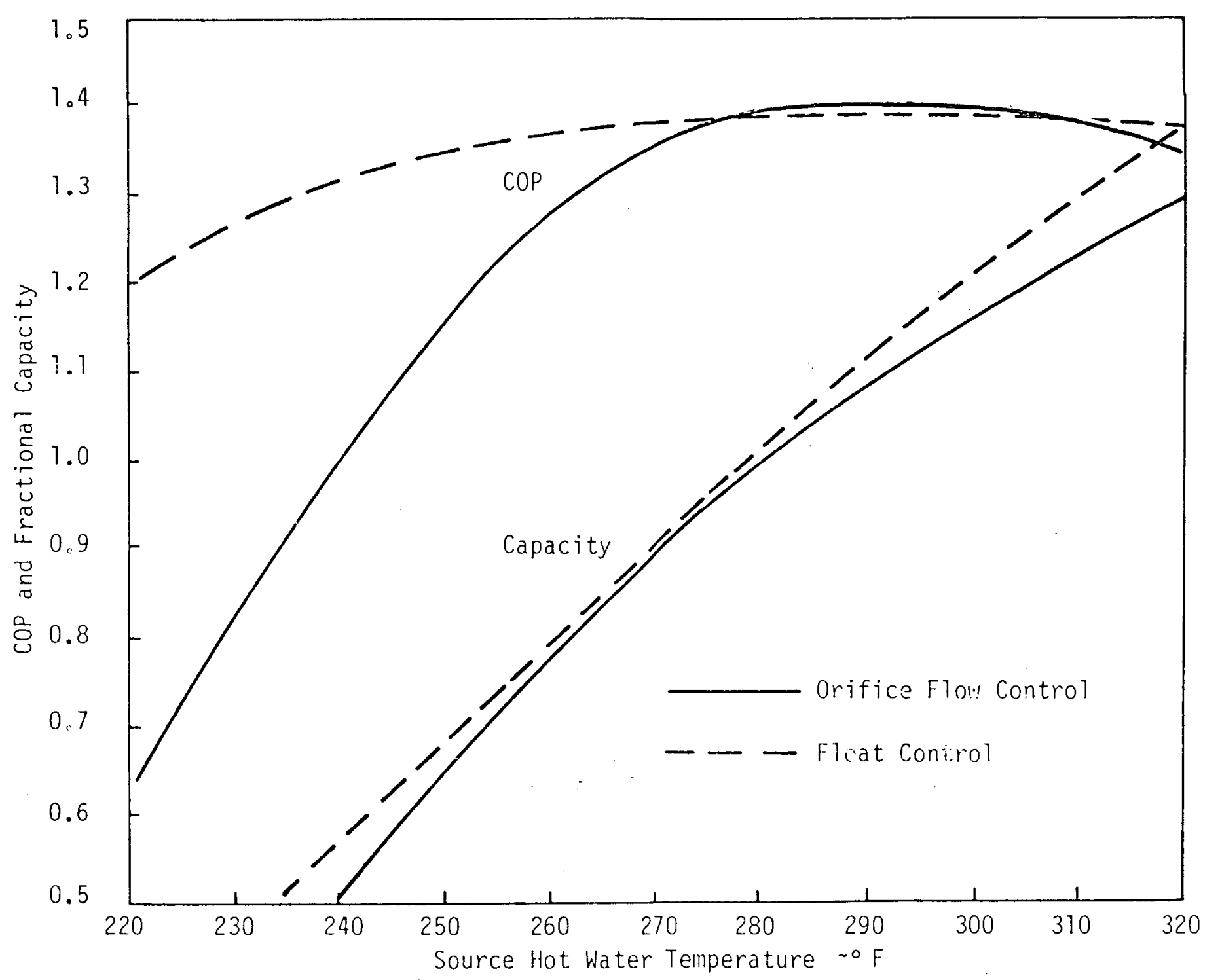

Figure 3.7 EFFECT OF ORIFICE FLOW CONTROL ON PERFORMANCE 


\subsubsection{Effect of Variable Solution Flow Rate}

All of the performance curves up to this point have been based on a constant solution circulation rate of $900 \mathrm{lb} / \mathrm{hr}$, (i.e., assuming a constant displacement pump). Figures $3.8 \mathrm{a}$ and $3.8 \mathrm{~b}$ show the effect (for float refrigerant control) of having solution pumps with the characteristics shown in Figure 3.9. Notice that at high operating temperatures, the capacity levels off in Figure 3.8a and actually turns downward in Figure 3.8b. As the operating temperature increases, the pressure differential against which the pump must work increases and, therefore, decreases the solution flow rate. This change is the opposite of what is desired at these higher operating temperatures. Figures $3.8 \mathrm{a}$ and $3.8 \mathrm{~b}$ also show as dashed curves the performance with constant solution circulation rate. Below the nominal condition of $280^{\circ} \mathrm{F}$ source hot water temperature, constant solution circulation results in better COP but slightly deteriorated capacity, while above $280^{\circ} \mathrm{F}$ the reverse is true. These effects should be considered when designing systems that have widely varying input source temperatures, such as solar input.

\subsubsection{Effect of Combined Orifice Flow Control and Variable Solution Flow Rate}

The curves of Figure 3.10a show the results of using an orificetype of flow control and a pump with characteristics of curve I in Figure 3.9. They follow fairly closely the performance curves of the system with the orifice-type of flow control at low temperatures (Figure 3.7), and follow the performance curves for pump I at higher temperatures (Figure $3.8 \mathrm{a}$ ). This is to be expected because of the 


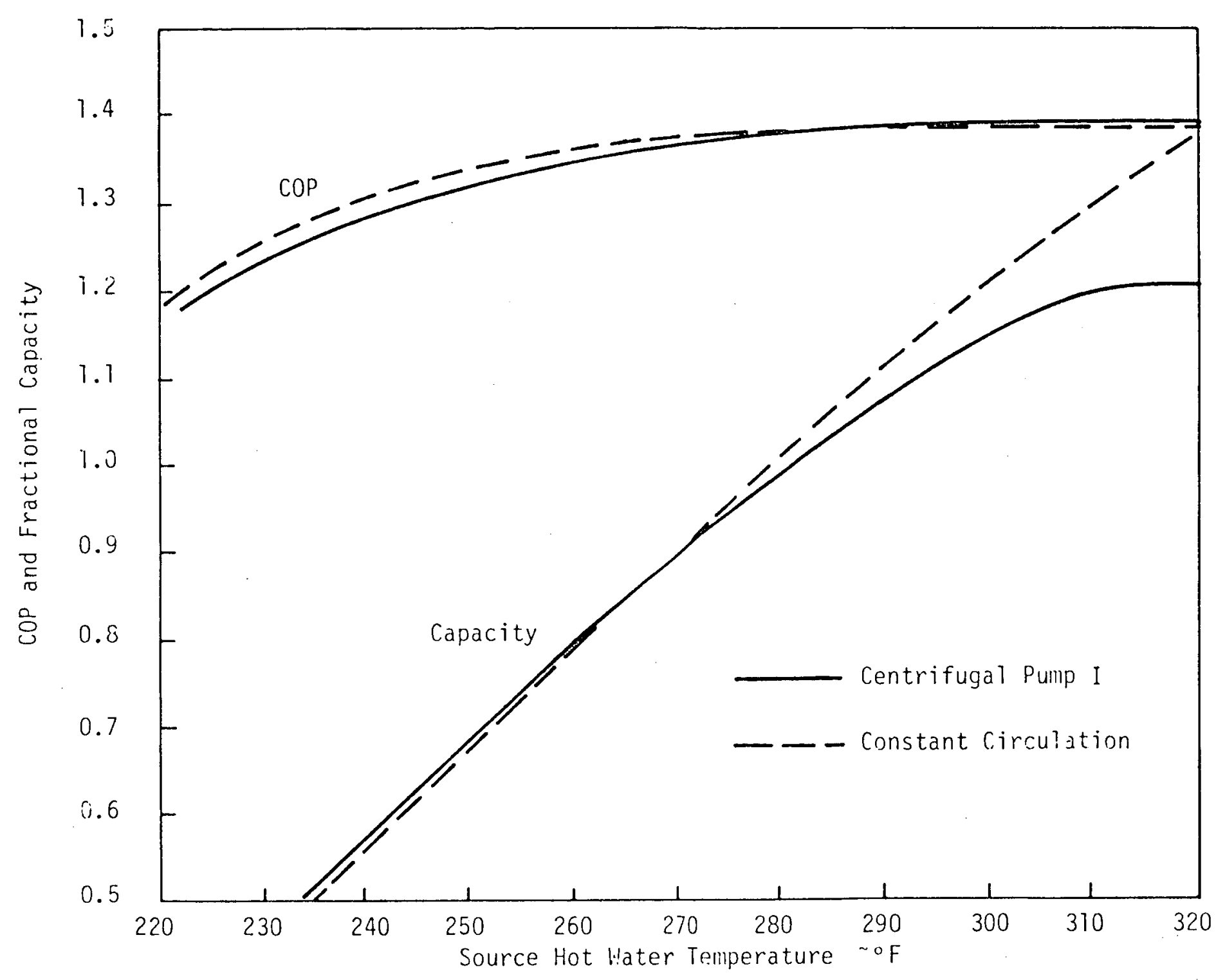

Figure 3.8a EFFECT OF CENTRIFUGAL PUMP I ON PERFORMANCE 


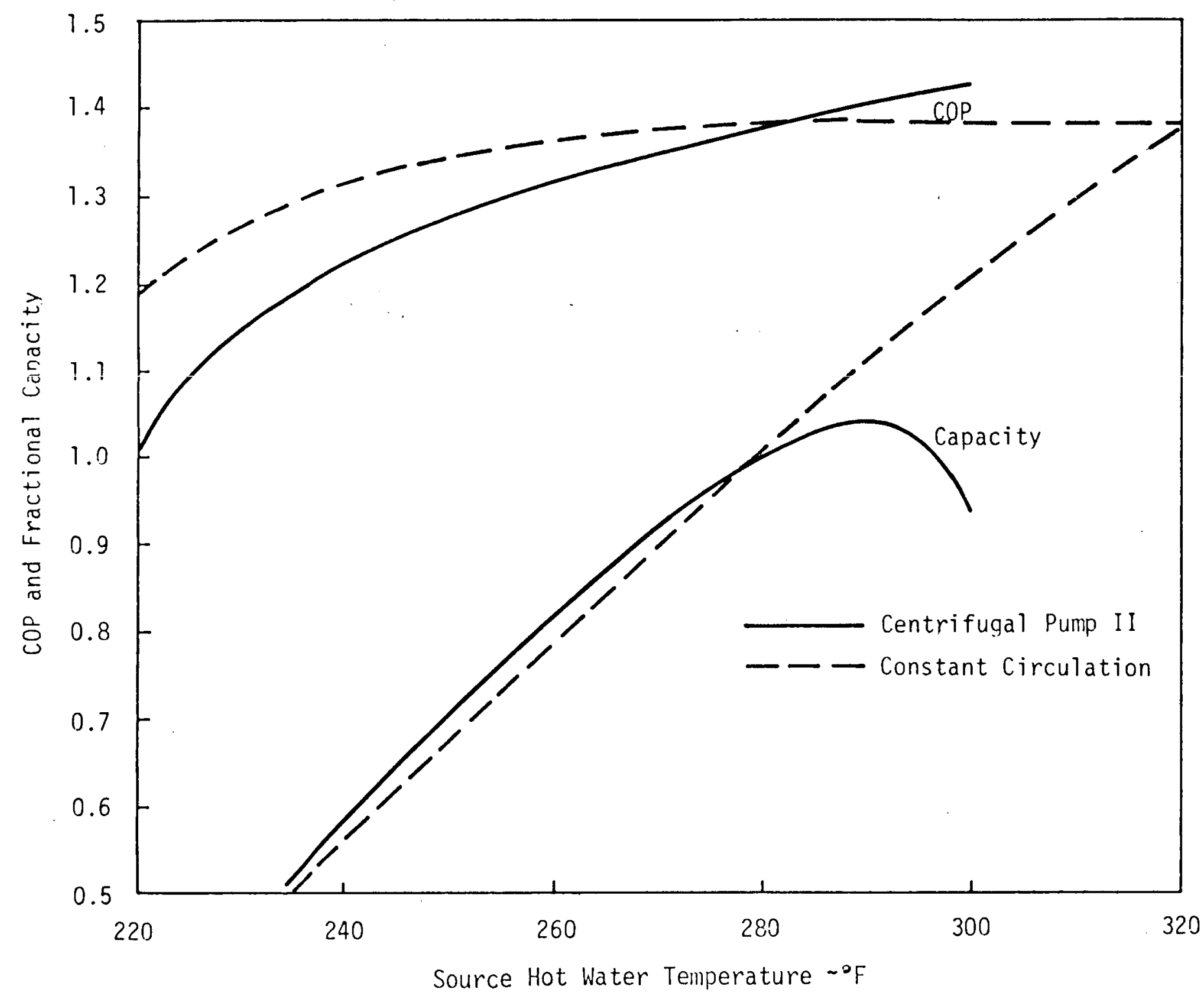

Figure 3.8b EFFECT OF CENTRIFUGAL PUMP II ON PERFORMANCE. 


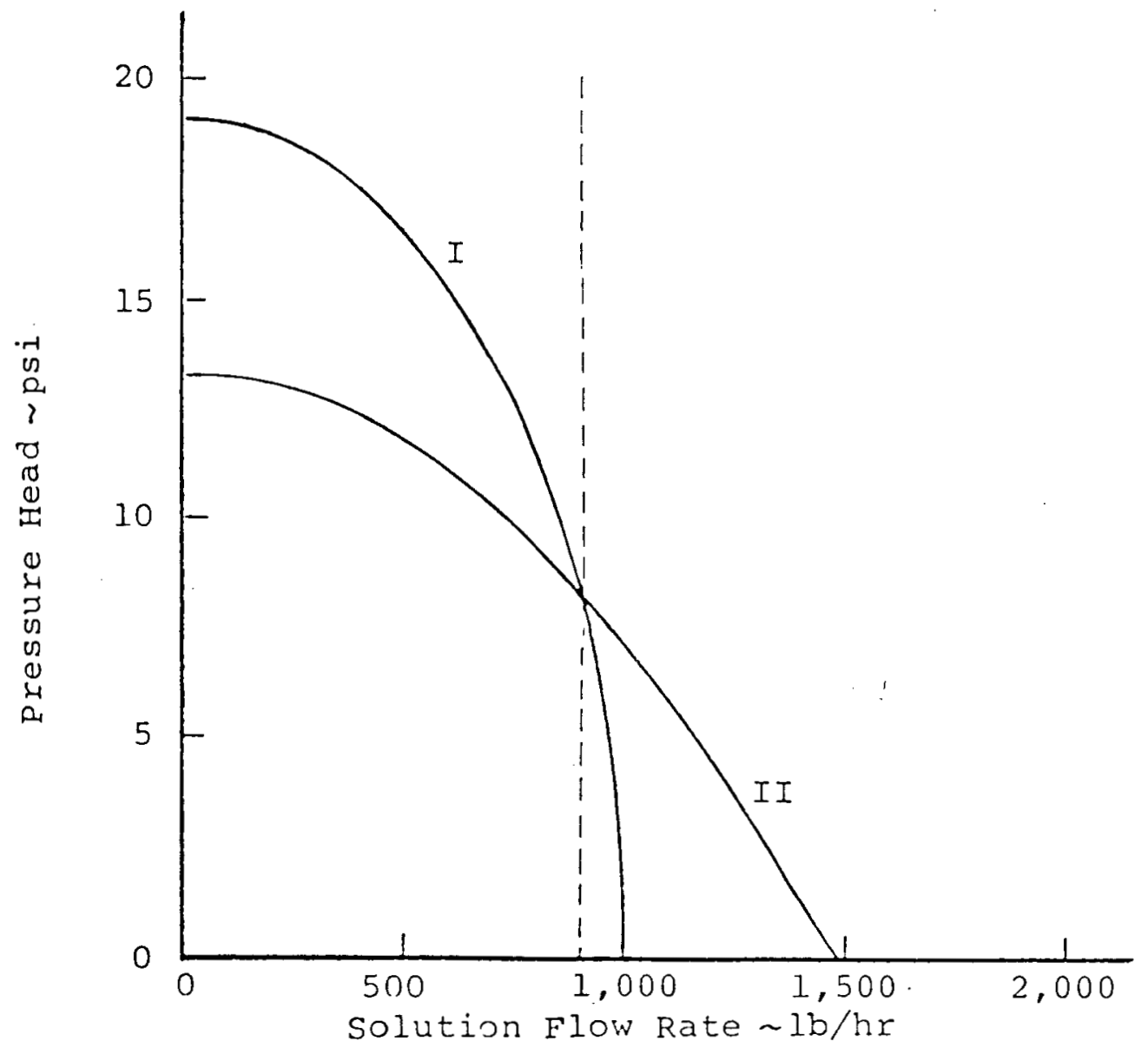

Figure 3.9 PUMP I AND II CHARACTERISTICS 


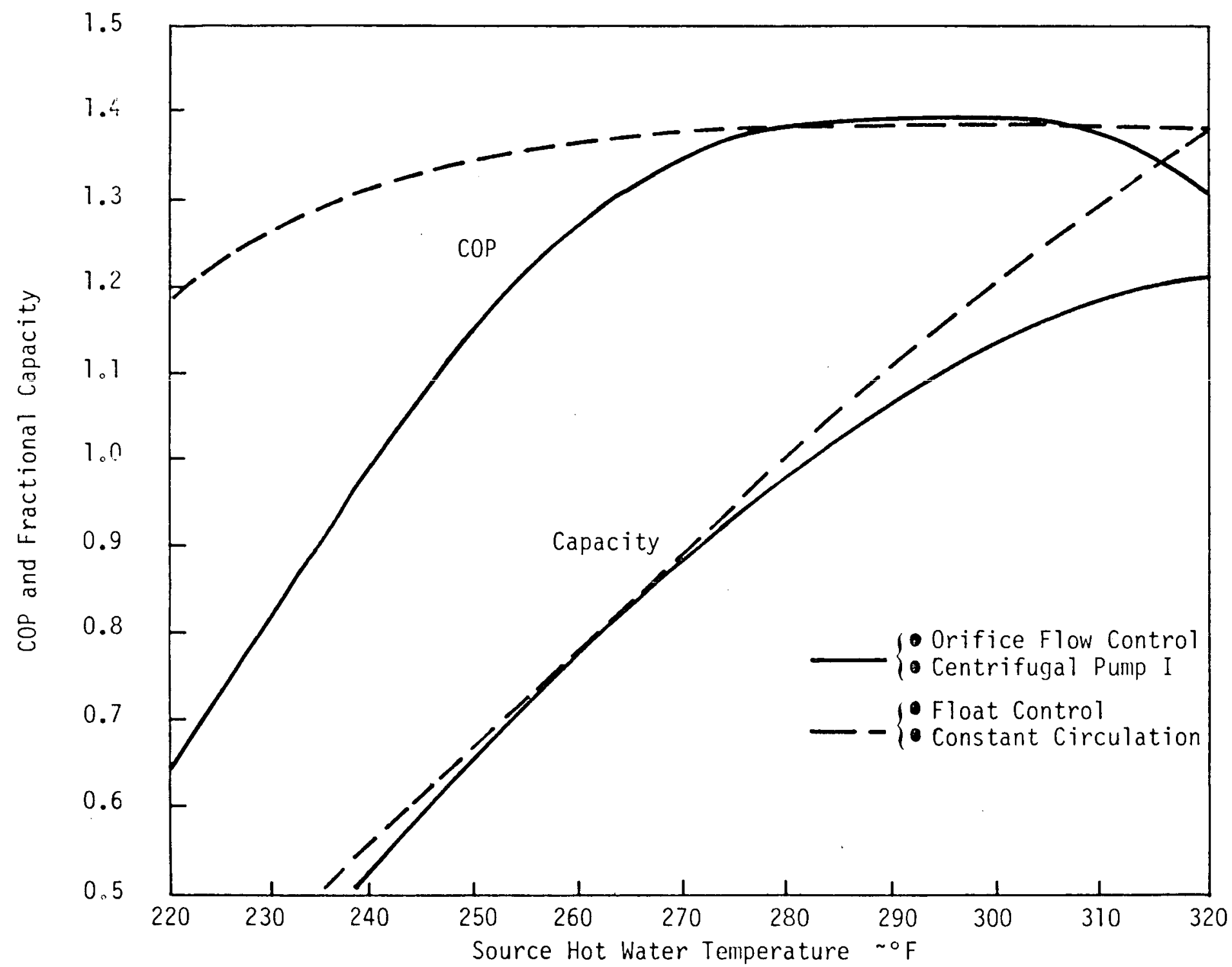

Figure 3.10a EFFECT OF ORIFICE FLOW CONTROL WITH CENTRIFUGAL PUMP I 
dominance of each of these variables in their respective regions. Thus the worst characteristics of each type of orifice control (pressure driven flow) and the pump characteristic are seen at the extremes of the range of source hot water temperatures.

Using the pump with curve II characteristics (Figure 3.9) in combination with orifice-type of flow control produces the curves in Figure 3.10b. This very sensitive pressure pump and orifice-type flow control give the worst performance of any condition examined. These types of curves, however, show performance very similar to real absorption machines.

\subsection{Performance Maps}

Three of the parameters examined previously--the source hot, cooling, and chilled water temperatures--are operational variables, while the remainder are selected design variables. It is of interest to observe how the system performance varies simultaneously with all three of these operational variables. The results discussed below are based on the nominal condition (including float control and constant circulation) except for variation in the three fluid temperatures.

Figures $3.17 a-c$ are general performance maps of the system, each the effect on performance of source hot and cooling water temperatures for a different chilled water temperature. They illustrate that these three variables can have a great impact on system COP and capacity. For example, if the cooling water temperature is decreased to $75^{\circ} \mathrm{F}$ and the chilled water temperature is increased to $60^{\circ} \mathrm{F}$, while the nominal $280^{\circ} \mathrm{F}$ source hot water temperature is maintained, the capacity of the system 


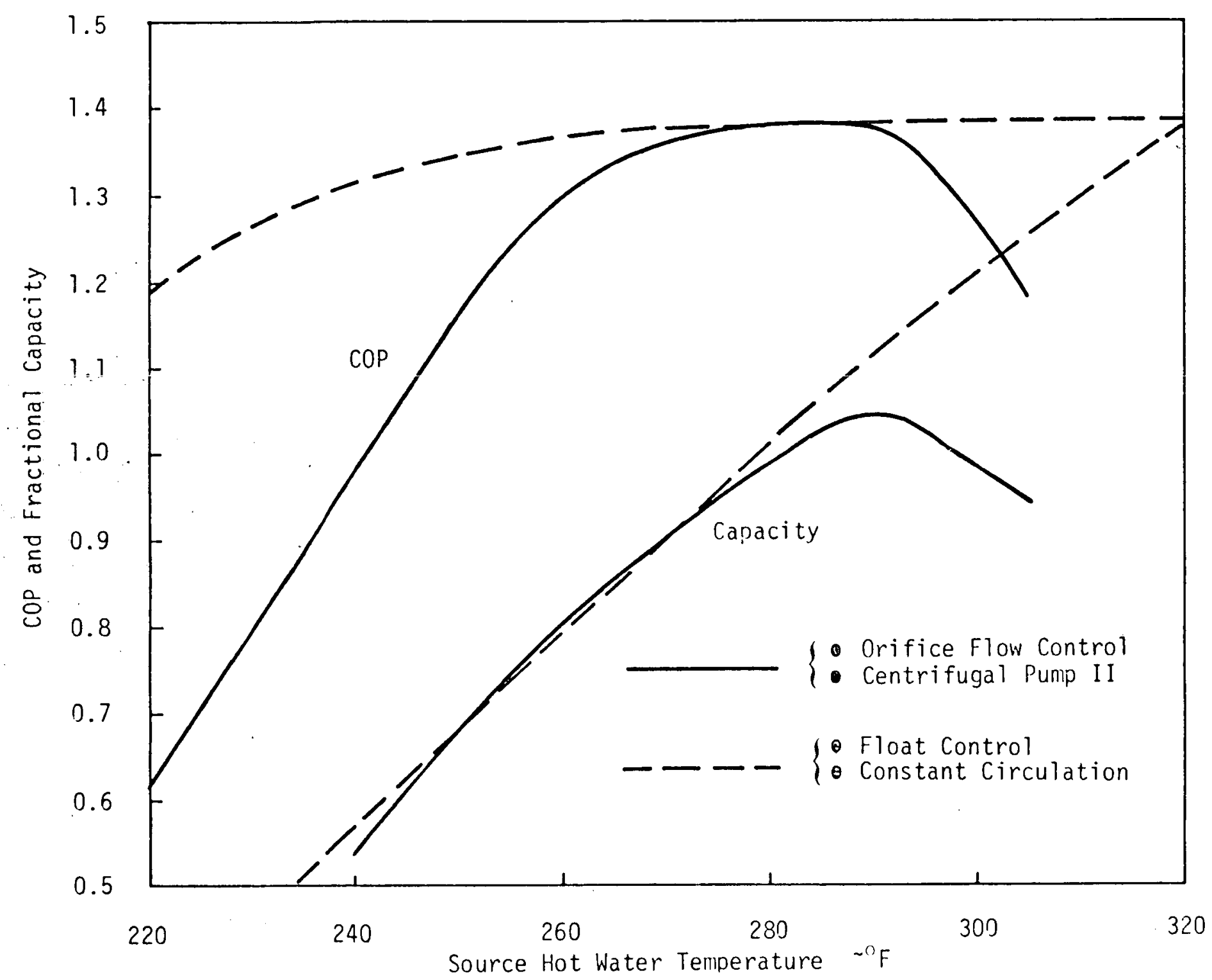

Figure $3.10 \mathrm{~b}$ EFFECT OF ORIFICE FLOW CONTROL WITH CENTRIFUGAL PUMP II 


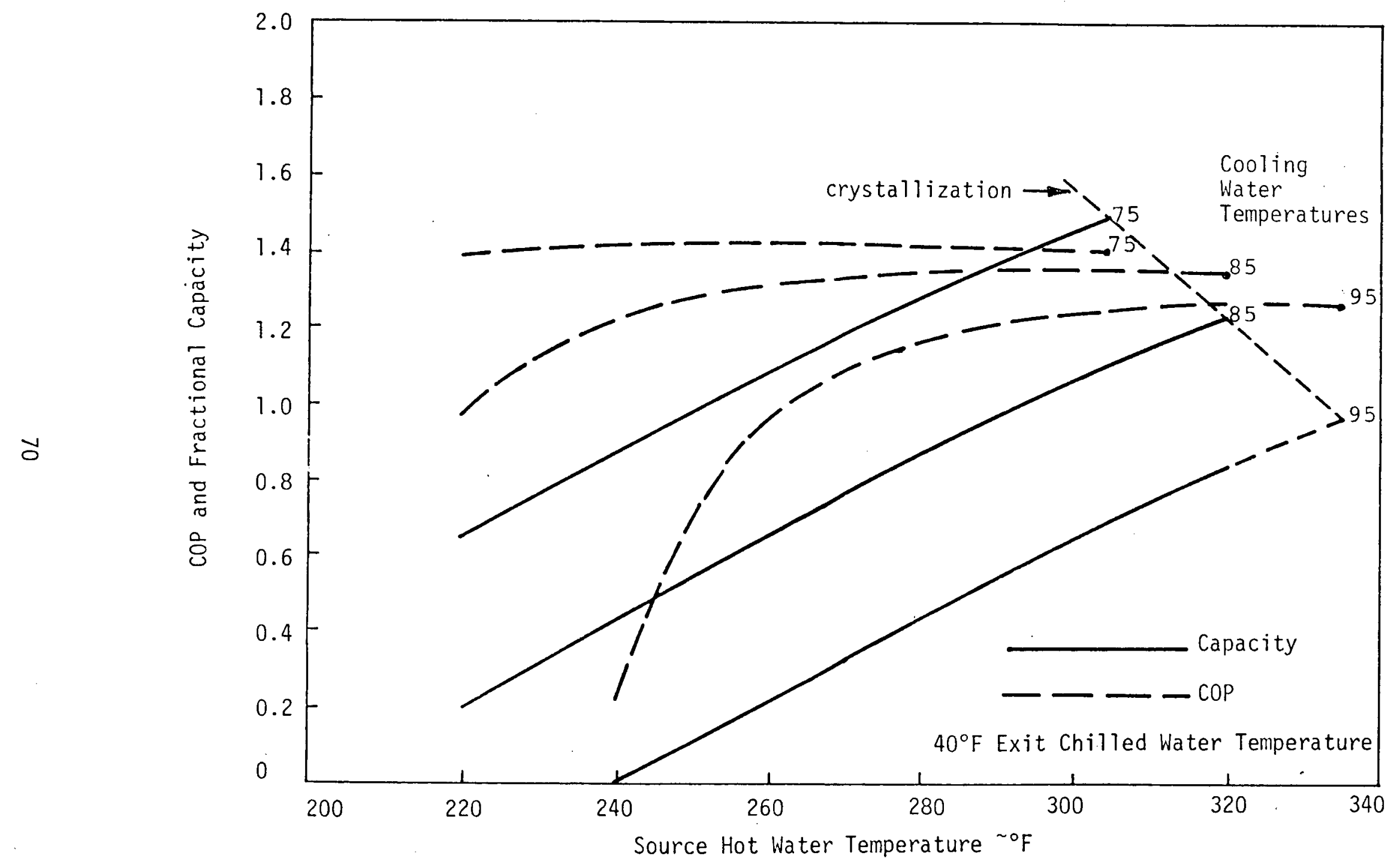

Figure $3.11 \mathrm{a}$ PERFORMANCE FOR $40^{\circ} \mathrm{F}$ EXIT CHILLED WATER 


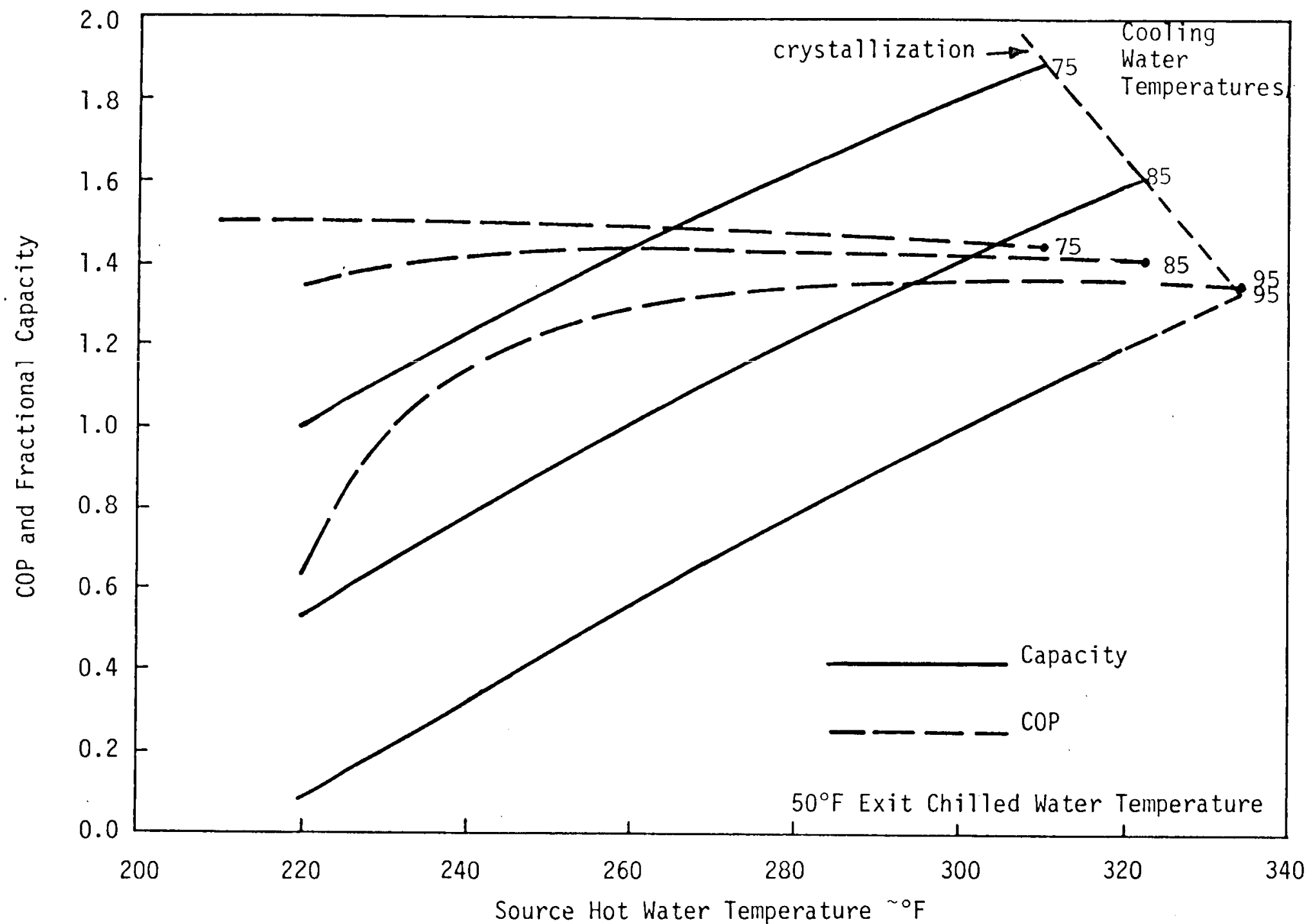

Figure $3.11 \mathrm{~b}$ PERFORMANCE FOR $50^{\circ} \mathrm{F}$ EXIT CHILLED WATER 


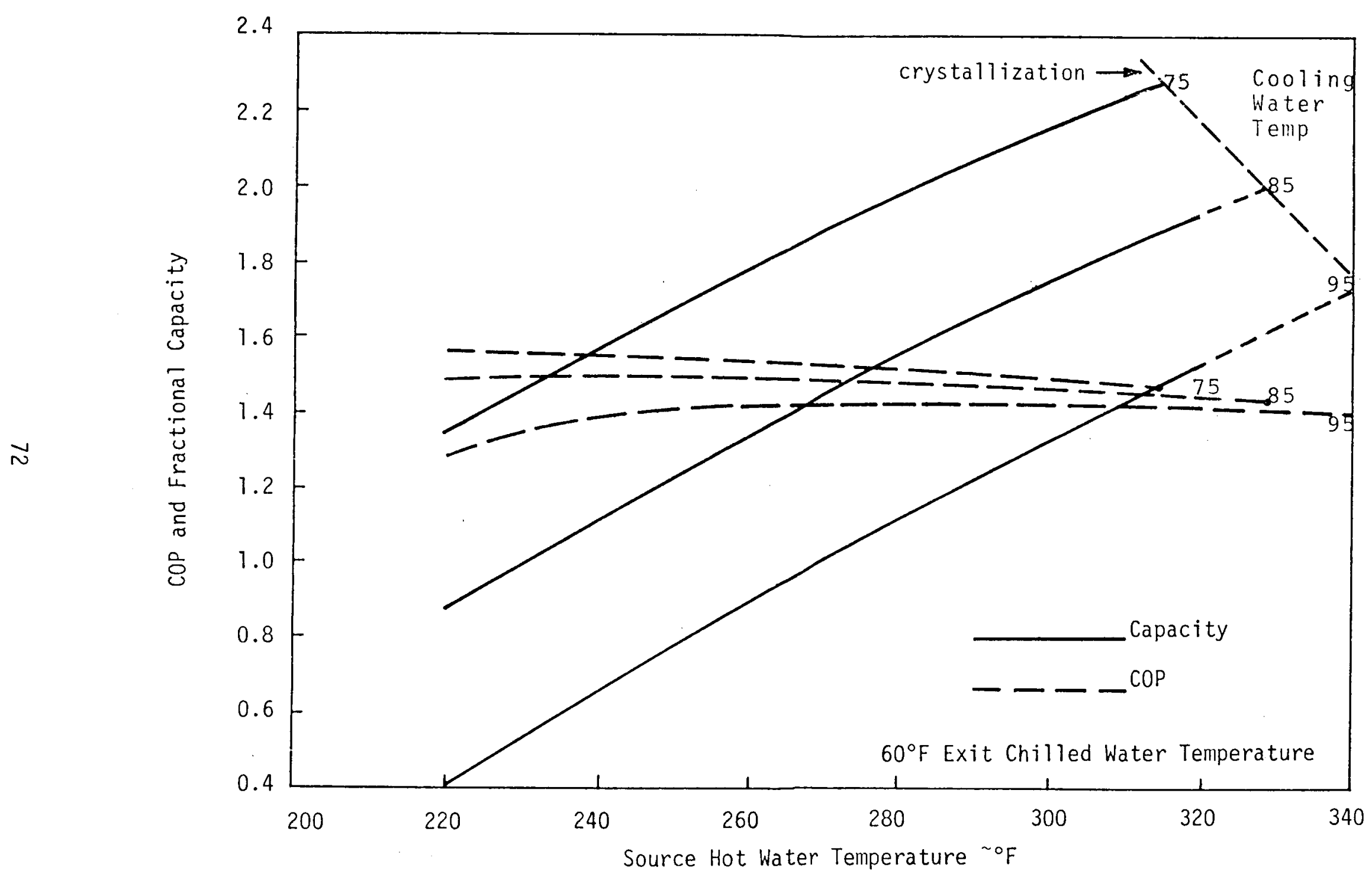

Figure $3.11 \mathrm{C}$ PERFORMANCE FOR $60^{\circ} \mathrm{F}$ EXIT CHILLED WATER 
can be nearly doubled and the COP increased to 1.5 compared to the nominal condition. Conversely, if the cooling water temperature is $95^{\circ} \mathrm{F}$, the chilled water exit temperature is $40^{\circ} \mathrm{F}$, and the source hot water temperature remains at: its nominal condition, then the capacity will drop $44 \%$ with a COP of only 1.16. It is encouraging to note, however, that the COP is still greater than 1.0 for most conditions in which an actual system might: operate.

\subsection{Comparison with Trane Two-Stage Absorption Unit Performance}

Although it was not an identified task in the contract objectives, some effort was directed toward predicting the performance of the Trane "Two-Stage Absorption Cold Generator" using the computer model, and comparing these results with the available data from Trane. This comparison is presented in Figure 3.12.

To simulate the performance of the Trane unit, all quoted flow rates and areas for the $385-$ ton machine were used $[19,20,21]$. The solution circulation rate was somewhat uncertain, as were the solution pump characteristics. The Trane unit is normally steam fired with 123 psig saturated steam; however, the equivalent performance is achieved with approximately $370^{\circ} \mathrm{F}$ hot water. The COP predicted by the model is presented in Figure 3.12. It is compared with the COP of the Trane unit, which is very close to 1.0 when operating at the $370^{\circ} \mathrm{F}$ design hot water condition. The prediction shows a COP approximately 10\% higher. The predicted and actual Trane capacity data presented in Figure 3.12 have been normalized by 385 tons, and thus represent fractional capacity. The predicted capacity at the design condition is approximately $30 \%$ 


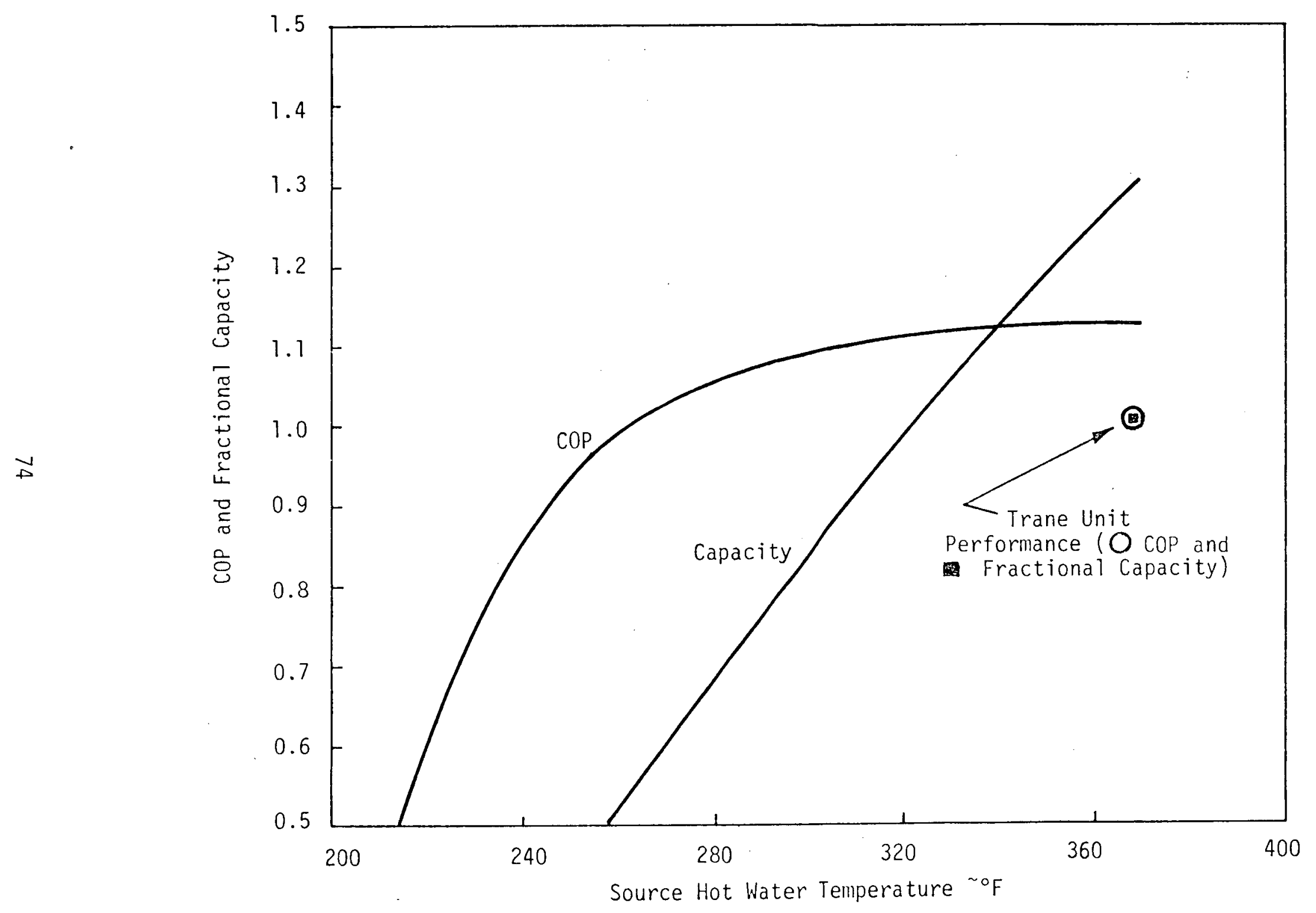

Figure 3.12 COMPARISON OF PREDICTED AND TRANE RESULTS 


\section{y.s.}

above the capacity of the Trane unit. The reason that data for the Trane unit are shown only for one temperature is that COP and capacity are not given as functions of hot water temperature.

The reasons for the predictions not being better are several. Only a modest effort was made to truly simulate the Trane unit's performance. Some specific differences are:

- The absorber heat transfer coefficient used was that recommended for another machine

- The Trane unit has an absorber recirculation pump

- The Trane unit has an evaporator recirculation pump

- More accurate heat exchanger tubing resistance and fouling factor data could be used

- The heat transfer coefficient data for water-lithium bromide solutions is extrapolated to heat fluxes below that for which the experimental data were obtained.

Considering the several sources of error and the limited effort expended, the comparison is reasonably good. The predicted COP is within 0.1 of the actual Trane performance, and earlier data showed that COP can be considerably greater than for the condition predicted; thus, the COP of the Trane unit could presumably be increased considerably.

\subsection{Cost Projections}

Figure 3.13 presents cost data for single- and double-effect absorption machines in the form of purchase price per ton as a function of machine tonnage. Cost data for single-effect machines were obtained from Arkla Industries, Yasaki, and Trane, while only data from Trane 


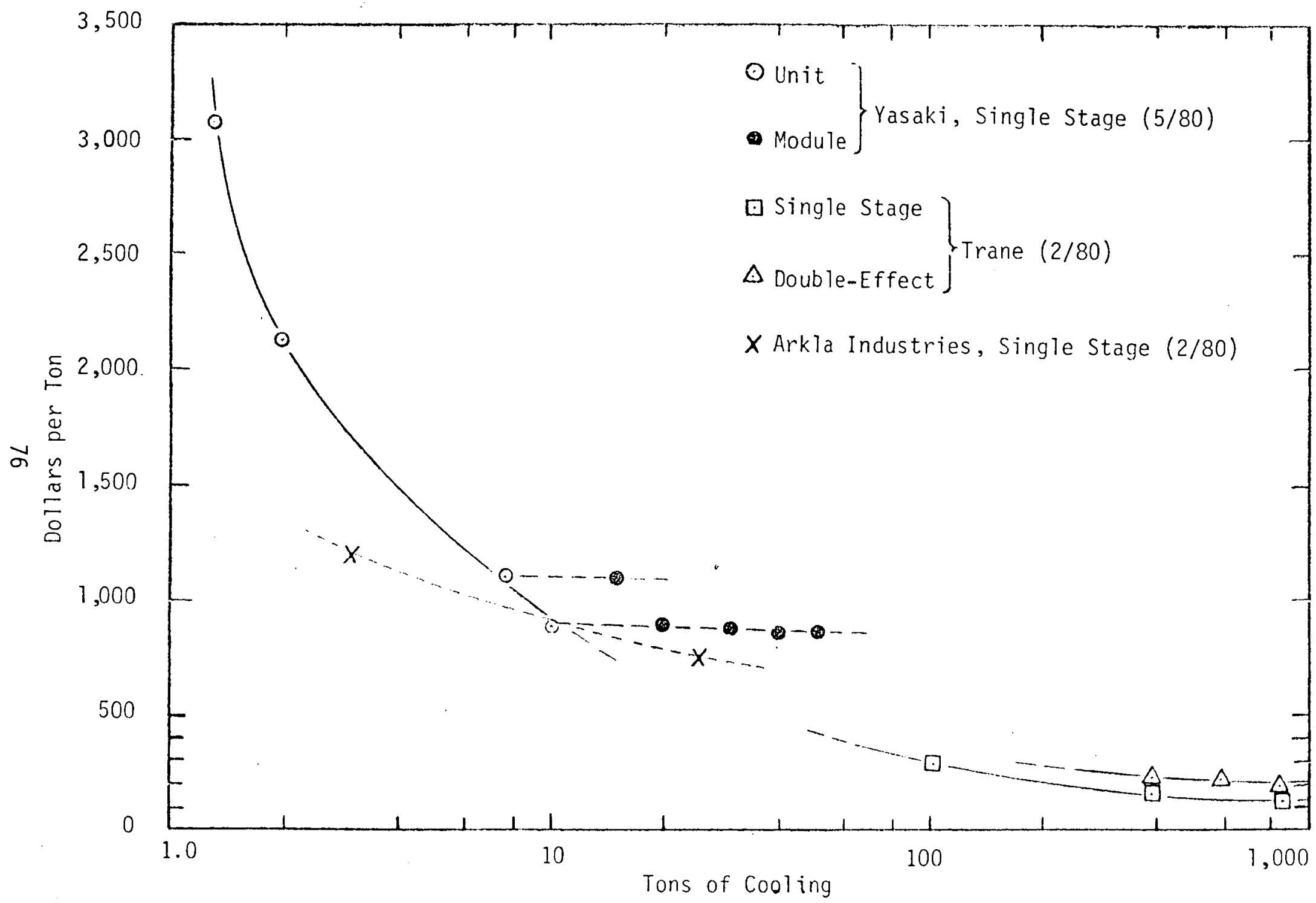

Figure 3.13 COMPARISON OF COST PER TON VERSUS TONNAGE FOR WATER-LITHIUM BROMIDE ABSORPTION SYSTEMS 
were available for double-effect machines, since at this date Trane is the only manufacturer of the latter. The single-effect units range in size from the 1.5-ton Yasaki units to the nearly 1,000-ton Trane units. Yasaki modularizes two of $i$ ts units to produce larger tonnage machines. The range of the Trane double-effect units is 385 to 1,000 tons. The Trane double-effect machines are approximately $70 \%$ more expensive than the single-effect for the same tonnage. It is anticipated that if manufactured in the 50 - to 100 -ton range the relative costs would be similar. Particularly in a solar cooling application where the collectors represent a large fraction of system cost, it would appear that doubleeffect machines would have potential merit. Even for other applications it would seem that double-effect machines would find favor because of current high fuel costs. 


\subsection{SUMMARY}

\subsection{Conclusions}

This study has developed a tool for use in analyzing the performance of double-effect absorption cooling systems and has examined the effect of the several design and operational variables on system performance. These latter results provide better insight into the effects of certain design features on the cycle operation. The major results include:

1. A computer model has been developed which simulates the dynamic behavior of double-effect water-lithium bromide absorption cooling systems.

2. The computer model was used to predict the performance of the Trane double-effect unit, and the results were found to be in fair agreement.

3. Double-effect systems can be operated at much lower temperatures than generally accepted. Most sources report that double-effect systems require hot water temperatures of 350 to $400^{\circ} \mathrm{F}$ to obtain good performance. This study indicates that for operation at source temperatures as low as approximately 230 to $240^{\circ} \mathrm{F}$, the COP is maintained remarkably flat and the penalty is mainly in capacity. The coefficients of performance for source hot water temperatures of $320^{\circ}, 280^{\circ}$ and $240^{\circ} \mathrm{F}$ are $1.372,1.376$, and 1.312 respectively, and the relative capacities are $1.37,1.0$, and 0.57 tons respectively. Coefficient of performance is quite constant, while capacity is nearly linearly dependent on source hot water temperature (in contrast to the Rankine cycle concept, where COP is strongly affected by source temperature). 
4. COPs approaching 1.5 can be obtained with favorable external conditions: chilled water temperatures above $50^{\circ} \mathrm{F}$ and cooling water temperatures lower than $80^{\circ} \mathrm{F}$.

5. The source hot water temperature, cooling water temperature, and chilled water temperature have strong effects on system performance. Capacity increases nearly linearly with hot and chilled water temperatures and decreases nearly linearly with cooling water temperature.

6. The flow rates of source hot water, cooling water, and chilled water have relatively small influences on coefficient of performance.

7. The sizes of the two liquid-liquid solution heat exchangers have a significant impact on system performance. Although the capacity of the system is not appreciably affected by them, there are significant increases of COP with increased heat exchanger area. Therefore, new double-effect systems should incorporate as much heat transfer surface area into these two heat exchangers as the economic constraints will a $110 w$.

8. The use of a float control valve (to insure that all refrigerant vapor from the first-effect generator is condensed in the second effect) is necessary to obtain maximum performance for units that will experience large variations in source hot water temperatures. This design feature would be highly desirable for solar-driven double-effect systems where the source temperature may vary strongly. The orifice-type of control, on the other hand, would be adequate for constant source temperature applications: and when very little on-off cycling is expected. 
9. The selection of the main solution pump is an important design consideration for absorption systems that operate with a wide range of source hot water temperatures. Ideally, for this type of application, a constant dispiacement pump or a variable rate pump with a control system that increases the flow with increasing pressure head is desirable. For systems that operate continuousiy and at a constant temperature, pump selection is not so critical.

10. Refrigerant pressure drop between the evaporator and absorber is an important design consideration. Often these components are in the same pressure vessel and a uniform pressure is considered throughout. But pressure drops of only $0.5 \mathrm{~mm}$ of mercury can cause a noticeable loss in system capacity, since the evaporator and absorber operate at only a few millimeters of mercury.

11. Cost data assembled for single- and double-effect machines suggest that in the 50- to 100-ton capacity double-effect:machines should cost about $70 \%$ more than single-effect machines of the same capacity.

\subsection{Recommendations}

It has been shown in several investigations that from a performance standpoint, double-effect absorption systems are more than competitive with conventional vapor. compression systems for the cooling of buildings using moderate grade thermal energy. The major drawback to the doubleeffect system is the cost. However, with rising costs of fossil fuel, the potentially high coefficients of performance make this system very attractive for geothermal and waste heat applications as well as for 
solar. More emphasis should be placed on optimizing the performance and expanding the applications of double-effect absorption systems.

The computer model, LIBR, developed in this study could be incorporated into a system simulation model for analysis and optimization of solar heating and cooling systems using double-effect absorption cooling. Simplified algorithms describing the performance of double-effect absorption systems should be developed from the results of program LIBR for use in large HVAC programs and for solar simulation programs such as TRNSYS. This capability would enable building and system designers to assess the relative merit of double-effect and single-effect systems.

Several additional tasks should be undertaken to further improve and justify the accuracy of the model. Specific effort should be devoted to comparing the predictions with the actual performance data of the Trane machines, and to improve the model where appropriate. While performance trends predicted by the model are felt to be valid, it is important for future users to know the quantitative accuracy of the results.

The current program, LIBR, should be a valuable design tool for either developing an absorption machine or modifying an existing design to optimize operation based on today's and future energy costs. The computer model developed under this study should be valuable in reevaluating the double-effect absorption system. Most of the available literature and the current hardware are from a time before the cost of energy was a primary concern. With the past and expected continuing escalation in energy costs, double-effect systems show real promise. 
Because of different design criteria brought on by rising energy costs, analytical tools like the model developed for this study can play an important part in developing a new generation of energy-efficient double-effect systems. 
APPENDIX A

COMPUTER PROGRAM LIBR 
PROGRAM LIBR (INPUT, OUTPUT, TAPES=INPUT, TAPEG=OUTPUT)

C

C

C

NOMENCLATURE

C

C VARIABLE

DESCRIPTION

C

C AABS

C ABM

C ACOND

C $A E$

C AGEN1

C AGEN2

C $A H \times 1$

C $A H \times 2$

C AI

C AL

C AN

C $\quad A R$

C CDM

c COX

C CHH

C CL

C $\mathrm{CN}$

C CONDG 1

C

CONDG 2

COP

CPH

$\mathrm{CH}$

CHC

CX()

DA

DABM

$D C$

DCDM

$D E$

DELP

DG 1

DG2

C DP 18

AREA OF THE ABSORBER

MASS OF SOLUTION INSIDE THE ABSORBER

AREA OF THE CONDENSER

AREA OF THE EVAPORATOR

AREA OF THE HIGH TEMPERATURE GENERATOR

AREA OF THE LOW TEMPERATURE GENERATOR

AREA OF THE LOH TEMPERATURE HEAT EXCHANGER

AREA OF THE HIGH TEMPERATURE HEAT EXCHANGER

FLOW AREA OF THE TUBES IN THE HIGH TEMPEPATURE

GENERATOR

ABSORBER TUBE LENGTH

NUMBER OF TUBES IN THE ABSORHER

OUTSIDE TO INSIDE AREA RATIO FOR ANY HEAT EXCHANGE

TUBE SURFACE

MASS OF FLUID IN THE CONDENSER

MAXIMUM YASS POSSIBLE. IN THE CONDENSER

CHILLED WATER FLOH RATE

CONDENSER TUBE LENGTH

NUMBE? OF TUBES IN THE CONDENSER

CONDUCTION TERM IN CALCULATION OF THE U.A. FOR THE HIGH TEMPERATURE GENERATOR

CONDUCTION TERM IN CALCULATION OF THE U.A. FOR THE

LOH TEMPERATURE GENERATOR

COEFFICIENT OF PERFORMANCE

SPECIFIC HEAT OF HATER

COOLING WATER FLOW RATE THROUGH THE ABSORBER

COOLING WATER FLOH RATE THROUGH THE CDNDENSER

CONCENTRATION AT SPECIFIC STATE POINTS

DIAMETER OF ABSORBER TUBES

DM/DT DERIVATIVE, THE CHANGE IN MASS IN THE ABSORBE?

HITH RESPECT TO TIME

DIAMETER OF THE CONDENSER TUBES

DMIDT DERIVATIVE, THE CHANGE IN YASS IN THE CONDENSER

WITH RESPECT TO TIME

DIAMETER OF THE EVAPORATOR TURE

PRESSURE DIFFERENTIAL OF PUMP

DIAMETER OF THE TUBE IN THE HIGH TEMPERATURE

GENERATOP

DIAMETER DF THE TUBE IN THE LOH TEMPERATURE GENERATOR TEMPORARY VARIABLE FOR CENTRIFUGAL PUMP FLOH 


\begin{tabular}{|c|c|c|}
\hline C & & CALCULATI ON \\
\hline C & DT & CHANGE IN TIME \\
\hline C & DT 1 & DT/DT DERIVATIVE, THE CHANGE IEN \\
\hline C & & TIME \\
\hline$C$ & DT 10 & THE CHANGE IN T(10) WITH RESPECT \\
\hline$C$ & DT 18 & THE CHANGE IN T(18) WITH RESPECT \\
\hline C & DT 4 & $\begin{array}{l}\text { TU TIME } \\
\text { OT/DT DERIVATIVE. }\end{array}$ \\
\hline$C$ & & TO TIME \\
\hline C & $\begin{array}{l}D \times A \\
0 \times 1\end{array}$ & IN X(1) WITH RESPECT \\
\hline C & & TO TI:AE \\
\hline $\begin{array}{l}\text { C } \\
C\end{array}$ & $0 \times 10$ & $\begin{array}{l}\text { DX/DT DER IVATIVE. } \\
\text { TO TIIE }\end{array}$ \\
\hline C & $0 \times 4$ & $\begin{array}{l}\text { DX/DT DERIVATIVE, THE CHANGE IN X(4) WITH RESPECT } \\
\text { TO TIME }\end{array}$ \\
\hline C & EFF 1 & FFECTIVENESS OF THE LOA TEMPERATURE HEAT EXCHANGER \\
\hline $\mathbf{C}$ & EFF2 & EFFECTIVENESS OF THE HIGH TEMPERATURE HEAT EXCHANGEZ \\
\hline C & EL & EVAPORATOR TUBE LENGTH \\
\hline C & EN & NUMBER OF EVAPORATOR TUBES \\
\hline C & FLWC) & FLOH AT EACH STATION \\
\hline C & FNR & TIME TO FINISH RUN \\
\hline C & F BC & TEMPORARY VARIABLE REPRESENTING THE FLOW AT STATION \\
\hline C & F $13 \mathrm{C}$ & INTERMEDIATE VALUE FOR FLW(13) \\
\hline C & F $8 \mathrm{~T}$ & ITERATIVE VARIABLE FOR THE FLOW AT STATION 8 \\
\hline C & GM1 & MASS OF SOLUTION IV THE HIGH TEMPERATURE GENERATOR \\
\hline C & GH2 & MASS OF SOLUTION IN THE LOH TEMPERATURE GENERATOR \\
\hline $\begin{array}{l}\text { C } \\
\text { C }\end{array}$ & GPP2 & $\begin{array}{l}\text { MASS FLOU RATE INSIDE THE TUBES OF LOH TEMPERATURE } \\
\text { GENERATOR }\end{array}$ \\
\hline $\begin{array}{l}\text { C } \\
\text { C }\end{array}$ & $6 \times 1$ & $\begin{array}{l}\text { AVERAGE CONCENTRATION IN THE HIGH TEMPERATURE } \\
\text { GENERATOR }\end{array}$ \\
\hline C & $6 \times 2$ & AVERAGE CONCENTRATION IN THE LOH TEMPERATURE \\
\hline C & & GENERATOR \\
\hline C & 61L & LENGTH OF TUBES IN THE HIGH TEMPERATURE GENERATOR \\
\hline C & G1 & NUMBER OF TUBES IN THE HIGH TEMPERATURE GENERATOR \\
\hline C & G2L & LENGTH OF THE TUBES IN THE LOH TEMPERATURE GENERATOR \\
\hline C & G2N & NUMBER OF TUBES IN THE LOH TEMPERATURE GENERATOR \\
\hline C & H() & ENTHALPY AT THE DESIGNATED STATION \\
\hline C & HD & MASS TRANSFER COEFFICIENT \\
\hline C & HF 14 & ENTHALPY. OF SATURATED LIQUID \\
\hline C & HF 20 & ENTHALPY OF SATURATED LIQUID AT STATION 20 \\
\hline C & HG Y 14 & ENTHALPY OF SATURATED VAPOR AT STATION 14 \\
\hline $\begin{array}{l}\text { C } \\
\text { C }\end{array}$ & HII & $\begin{array}{l}\text { INSIDE HEAT TRAYSFER COEFFICIENT FOR THE HIGH } \\
\text { TEMPERATURE GENERATOR }\end{array}$ \\
\hline $\begin{array}{l}C \\
C\end{array}$ & $\mathrm{HI2}$ & $\begin{array}{l}\text { INSIDE. HEAT TRANSFER COEFFICIENT FOR THE LOH } \\
\text { IEMPERATURE GENERATOR }\end{array}$ \\
\hline C & HOL & OUTSIDE HEAT TRANSFER COEFFICIENT FOR THE HIGH \\
\hline C & & TEMPERATURE GENERATOR \\
\hline C & HO2 & DUTSIDE HEAT TRANSFER CDEFFICIENT FOR THE LOW \\
\hline C & & TEMPERAT URE \\
\hline$C$ & H14 & SATURATED LIQUID \\
\hline
\end{tabular}




\begin{tabular}{|c|c|c|}
\hline C & I & DUMAY VARIABLE \\
\hline c & ICHAR & ARRAY FOR TITLE IN PLOTTING ROUTINE \\
\hline C & IER & FLAG FROM PLOTTING ROUTINE \\
\hline C & IFF & FLAG FOR CONSTANT DISPLACEMENT PUMP OR CENTRIFUGAL \\
\hline C & & PUMP OPTION \\
\hline C & & $(0=C E N T$ PUMP, 1 = CONSTANT DISPLACEMENT, \\
\hline C & IFLO & FLAG FOR REFRIGERANT FLOH CONTROL OPTION \\
\hline & & $(0=$ FLOAT CONTROL ; 1 = ORIFICE CONTROL, \\
\hline C & IMAG 4 & \\
\hline C & I NC & VARIABLE REQUIRED FOR USPLT RDUTINE \\
\hline C & I 0 & INTEGRATION ORDER (LINEAR ALWAYS USED) \\
\hline c & I OPT & PARAMETER REQUIRED FOR USPLT ROUTINE \\
\hline c & I STEPS & NUMBER OF STEPS PER HOUR (I/OT) \\
\hline C & IY & PARAMETER REQUIRED FOR USPLT POUTINE \\
\hline C & $\mathcal{J}$ & NUMBER OF POINTS STORED FOR PLOTS \\
\hline C & JA & TITLE ARRAY FOR PLOTTING ROUTINE \\
\hline C & $J B$ & COUNTER FOR JA \\
\hline C & $J N$ & \\
\hline C & JS & \\
\hline C & JS4 & \\
\hline C & JU & LOOP COUNTER \\
\hline C & JUU & LOOP COUNTER \\
\hline c & K & \\
\hline C & KC & COUNTER FOR TIME INTERVAL FOR PRINTING \\
\hline C & KJ & FLAG FOR PRINTING INTERVAL \\
\hline C & KK & \\
\hline c & KL & \\
\hline C & KP & CCUNTER FOR TIME INTERVAL FOR PLOTTING \\
\hline C & LC & LOJP COUNTER FOR T(20) LOOP \\
\hline C & LH & LOOP COUNTER FOR T(8) LOCP \\
\hline C & LIM & MAXIMUM LIMIT FOA IMPLICIT LOOPS \\
\hline C & LL & LOOP COUNTER FOR SUBROUTINES ABST AND GEN \\
\hline C & $C P$ & LOOP COUNTER FOR TCP LOOP \\
\hline C & LPC & LDOP COUNTER FOR PRINT STATEMENTS \\
\hline C & L13 & LOOP COUNTER FOR FLW(13) LOOP \\
\hline C & L8 & LOOP COUNTER FOR FLH( \&) LOOP \\
\hline C & M & INDEX FOR READING TITLES FOR PLOTS AND PARAMETER FOR \\
\hline C & & USPLT ROUTINE \\
\hline C & NC & CONVERGENCE PARAMETER FROM CONV ROUTINE ( T(20) LODP) \\
\hline C & NCURVES & PARAMETER FOR CECPLOT ROUTINE \\
\hline C & NF & FLAG FOR END OF PROGPAM IN SUBROUTINE PRNTF \\
\hline C & NH & CONVERGENCE PARAMETER FROM CONV POUTINE ( T(B) LOOP, \\
\hline C & NP & CONVERGENCE PARAMETER FROM CONV ROUTINE (TCP LOOP, \\
\hline C & NPTS & PARAMETER FOR CECPLOT - NUMBER OF POINTS \\
\hline C & N13 & CONUERGENCE PARAMETER FROM CONV ROUTINE \\
\hline C & & (FLH(13) LOOP) \\
\hline C & M8 & CONVERGENCE PARAMETER FOR. CONV ROUTINE \\
\hline C & & $(F L W(B) \quad L O O P)$ \\
\hline C & $P()$ & PRESSURES AT DESIGNATED STATICN \\
\hline C & P I & $P I=3.1415926$ \\
\hline C & PR & PRANDTL NUMBER INSIDE THE TUBES OF THE HIGH \\
\hline C & & TEMPERATURE GENERATOR \\
\hline C & PR I & PRINT INTERVAL \\
\hline
\end{tabular}




\begin{tabular}{|c|c|}
\hline C & QABS \\
\hline C & QCOND \\
\hline C & QEVAP \\
\hline C & QGEN1 \\
\hline C & QGEN2 \\
\hline C & $\theta x$ \\
\hline C & RE 1 \\
\hline C & RG \\
\hline C & S() \\
\hline C & SC \\
\hline c & STR \\
\hline C & $S H$ \\
\hline C & \\
\hline C & SHPT \\
\hline C & $T()$ \\
\hline C & $\mathrm{TCP}$ \\
\hline C & TCPC \\
\hline C & $T C P P$ \\
\hline c & TCPT \\
\hline C & $\mathrm{TCH}$ \\
\hline C & $T E$ \\
\hline C & TEP \\
\hline C & $T G$ \\
\hline C & $T G_{1}$ \\
\hline c & TG2 \\
\hline C & $T M$ \\
\hline C & TPRINT \\
\hline C & TSM \\
\hline$c$ & \\
\hline C & $T 12$ \\
\hline C & $T 14$ \\
\hline$c$ & $T 20 \mathrm{C}$ \\
\hline C & T20T \\
\hline C & UAGEN 1 \\
\hline C & \\
\hline C & UAGEN2 \\
\hline C & \\
\hline c & UAHX 1 \\
\hline C & UAHX2 \\
\hline C & V( ) \\
\hline C & $x()$ \\
\hline C & $\times A C$ \\
\hline C & XB \\
\hline C & $x \times(\quad)$ \\
\hline C & $x \times x<$, \\
\hline C & $X X Y()$ \\
\hline C & $\times 14$ \\
\hline C & $Y()$. \\
\hline C & Y11) \\
\hline c & $Y 2()$ \\
\hline C & Y36 \\
\hline c & $Y 4(1)$ \\
\hline C & Y5() \\
\hline C & Y G ( ) \\
\hline c & ZRHO \\
\hline
\end{tabular}

HEAT TRANSFER IN THE ABSORBER

HEAT TRANSFER IN THE CONDENSER

HEAT TRANSFER IN THE EVAPORATOR

HEAT TRANSFER IN THE HIGH TEMMPERATURE GENERATOR

HEAT TRANSFER IN THE LOH TEMPERATURE GENERATOR

OUALITY AT STATION 20

REYNOLDS NUMBER IN THE FIRST GENERATOR IN THE TUBES

TITLE VARIABLE FOR PLOTS

ENTROPY RETURNED FROM SUBROUTINE 'SUPER'

AHOUNT OF SUB-CDOLING

STARTING TIME

SOURCE WATER FLOA RATE TO THE HIGH TEMPERATURE

GENERATOR

SOURCE HATER PER TUBE

TEMPERATURE AT THE INDICATED STATION

COOLING WATER TEMPERATURE EXITING FROM THE ABSJRBER

TEMPORARY VARIABLE FOR TCP

COOLING WATER TEMPERATURE EXITING FROM THE CONDENSER

TEMPORARY VARIABLE FOR TCP

EMTERING COOLING HATER TEMPERATURE TO THE ABSCRBER

CHILLED WATER EXITING TEMPERATURE

ENTERING CHILLED WATER TEMPERATURE

A VERAGE SOURCE HATER TEMPERATLRE $((T G 1+T$ T2)/2)

ENTERING SORCE WATER TEMPERATURE

EXITING SCURCE HATER TEMPERATURE

TIME

TOTAL SUM OF THE MASS IN COMPCNENTS THAT HAVE A LIBR-IATER SOLUTION

EQUILIBRIUM TEMPERATURE AT STATION 12

SUB-COOLED TEMPERATURE AT STATION 14

TEMPORARY VARIABLE FOR THE TEMPERATURE AT STATION 20

TEMPORARY VARIABLE FOR THE TEMPERATURE AT STATION 20

OVERALL HEAT TRANSFER COEFFICIENT OF THE HIGH

TEMPERATURE GENERATOR

UVERALL HEAT TRANSFER COEFFICIENT OF THE LOH

TEMPERATURE GENERATOR

UA OF THE LOW TEMPERATURE HEAT EXCHANGER

UA OF THE HIGH TEMPERATURE HEAT EXCHANGER

SPECIFIC VOLUME RETURNED FROM SUBROUTINE ' SUPER・

AVERAGE CONCENTRATION OF SOLUTION IN THE SYSTEM

ARRAY FOR PLOTTING OF TG1
ARRAY FOR PLOTTING OF TM
QUALITY AT STATION 14
ARRAY FOR PLOTTING
ARRAY FOR PLOTTING T(1)
ARRAY FOR PLOTTING T(4)
ARRAY FOR PLOTTING T( 9$)$
ARRAY FOR PLOTTING CX(1)
ARRAY FOR PLOTIING CX(4)
ARRAY FOR PLOTTING CX(3)
DENSITY OF HATER INSIDE TUBES OF SECONO GENERATOR 


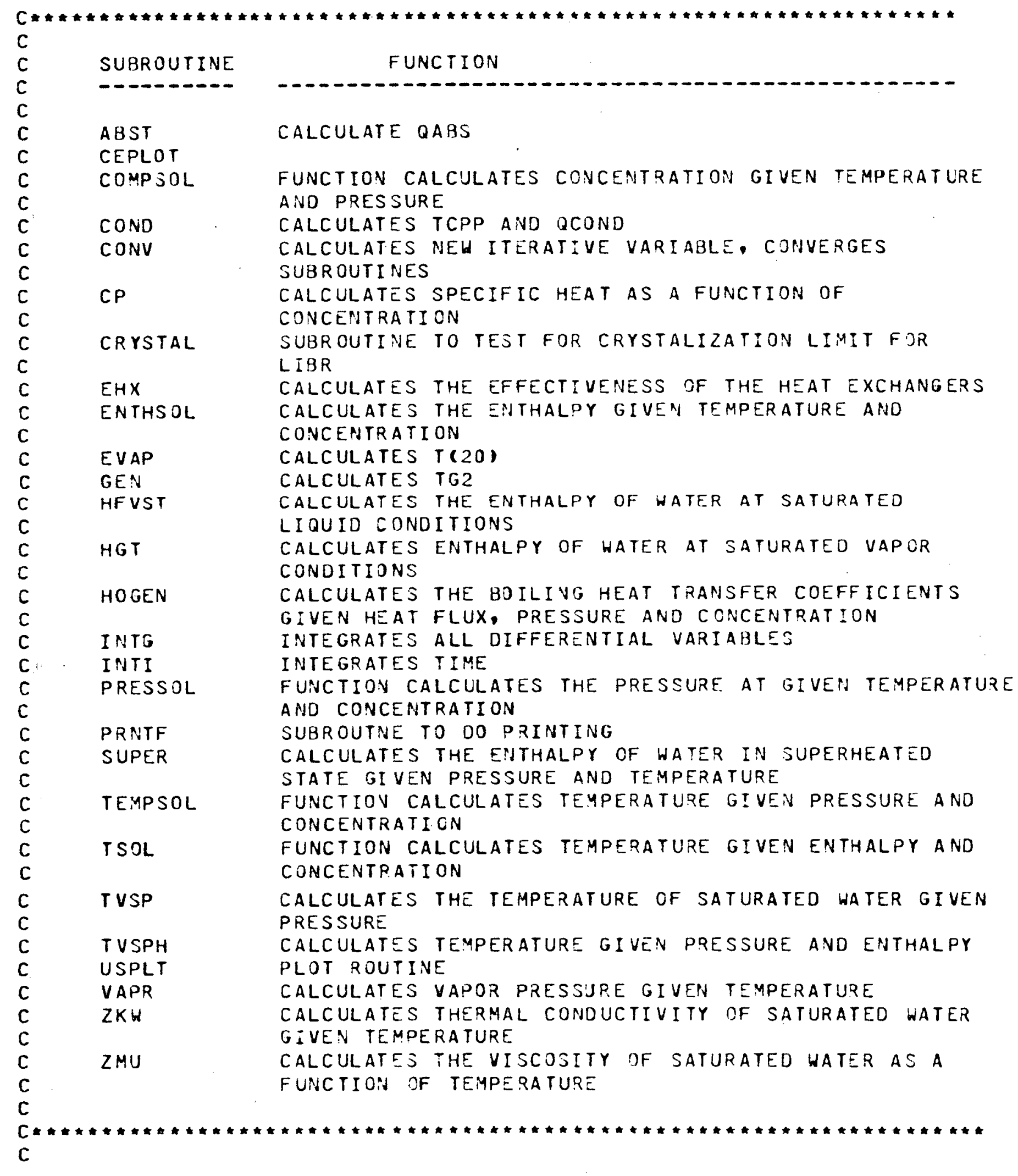




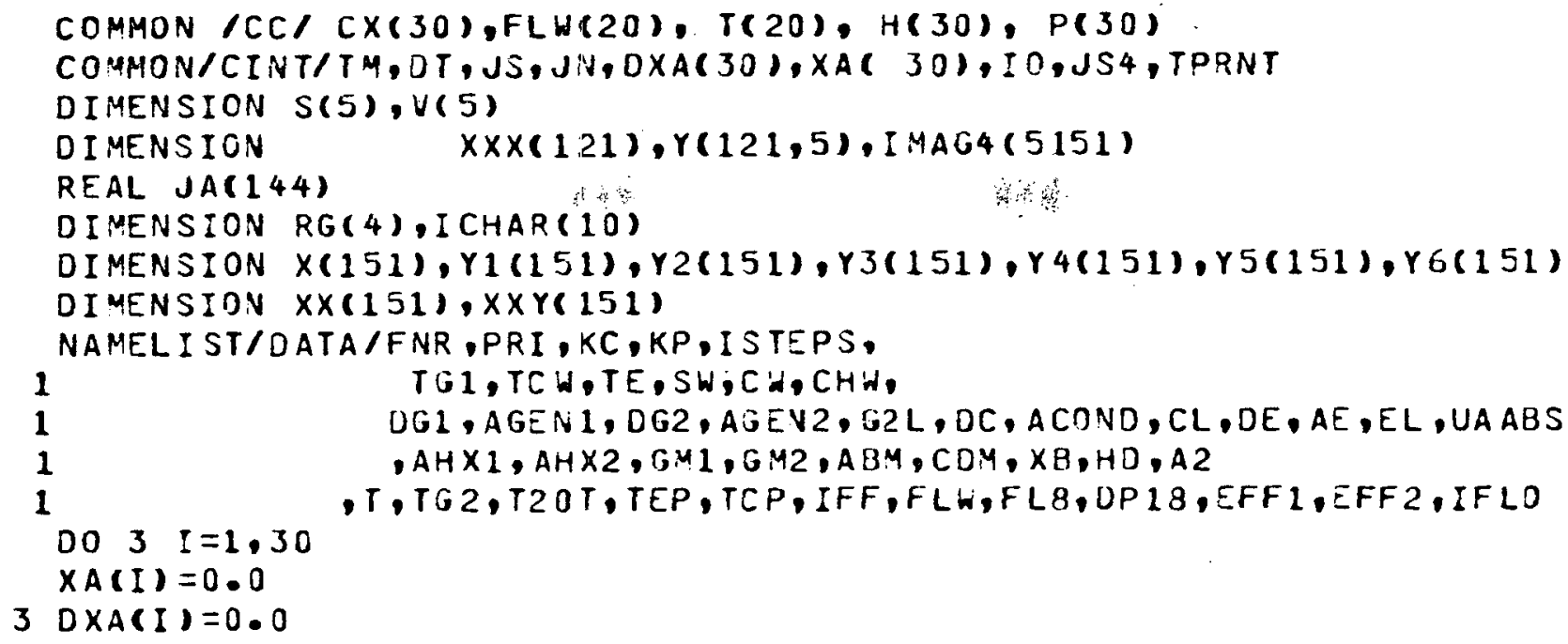




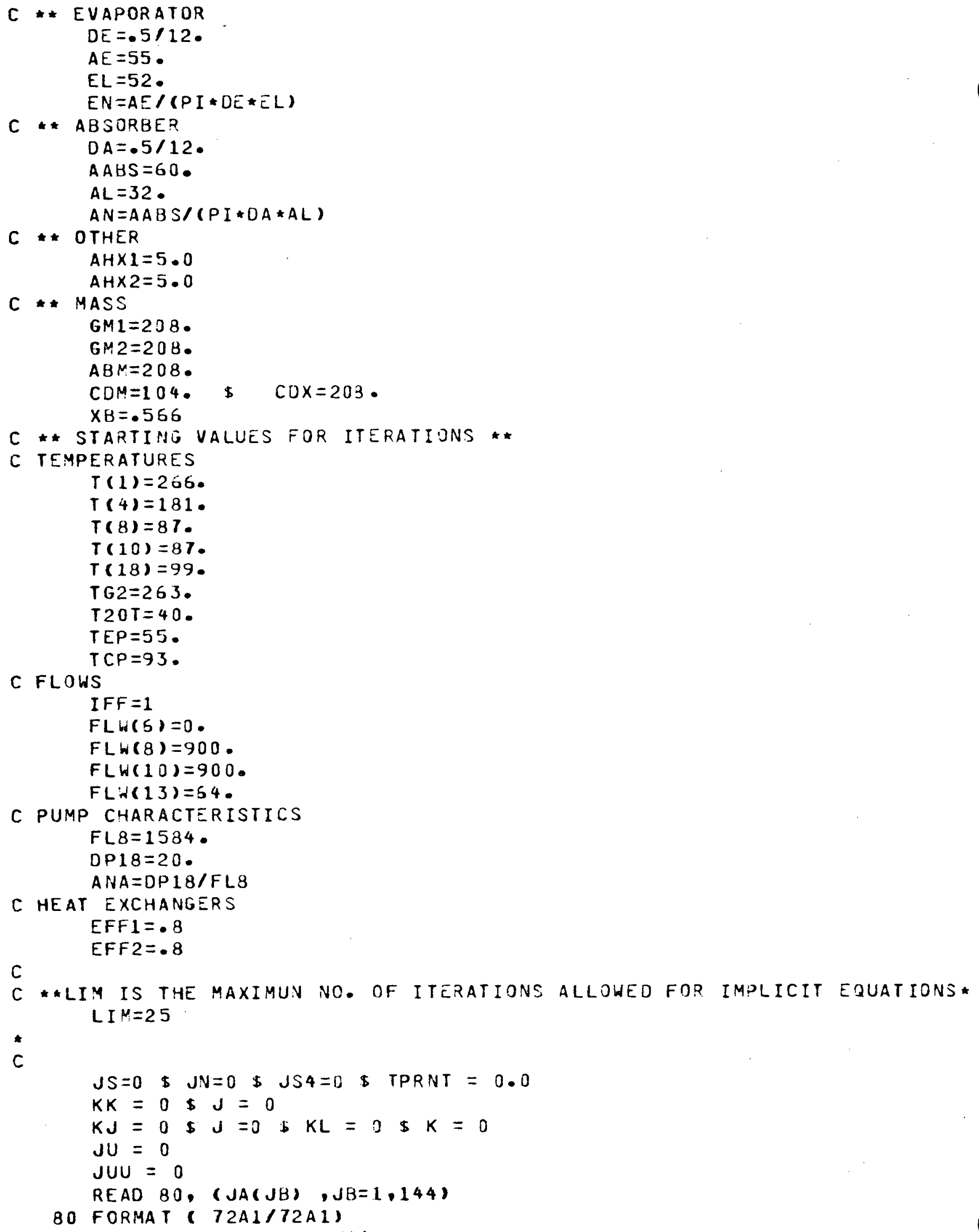

*

JS $=0$ \$ JN=0 \$ JS4 $=0$ \$ TPRNT $=0.0$

$K K=0$ \& $J=0$

$K J=0 \$ J=0 \quad \& L=0 \$ K=0$

$J U=0$

JUU $=0$

READ $80,(J A(J B), J B=1,144)$

80 FORMAT (72A1/72A1) 
READ $81,(R G(I), I=1,4)$

81 FORMAT (\$F10.0)

READ $82,(I$ CHAR (I), I $=1,2)$

82 FORMAT (1OA1)

C

READ ( 5, DATA)

IF (EOF (5). NE.O) GO TO 4

4 CONTINUE

$T S M=G M 1+G M 2+A B M$

$C \times(1)=X B$

$C \times(4)=X B+.04$

$C \times(10)=(T S M * X B-(C X(1) * G M 1)-(C X(4) * G M 2)) / A B H$

$C \times(8)=C \times(10)$

C OTHERS

$C W C=C H$

$T C P T=T C P$

QGEN1 $=S W *(T G 1-T G 2)$

QGEN2 $=.8 *$ OGEN1

$F L W(18)=.10 * F L W(10)$

$T C P P=T C P+3 \cdot 5$

WRITE (6,DATA)

PRINT 6O0,STR,FNR,DT,PRI,KC,KP

600 FORMAT (1H1./,1X, *STR=* ,F4.2, $6 X, * F N R=*, F 5.2,6 X, * D T=* F 4.3,6 X$,

$1 * P R I=*, F 4,3,6 X, * K C=*, I 3,9 X, * K P=*, I 3 / 1$

PRINT 601,TGL,TCH,TE, SH, CH,CHW

601 FORMAT $\left(1 X_{0} * T G 1=*, F 5.1,5 X, * T C H=*, F 5.1,6 X_{*} * T E=*, F 5.1,5 X, * H=*\right.$,

IF $5.0,6 X, * C H=*, F G .0,6 X, * C H W=*, F 7.0 /)$

PRINT 602,DG1, AGEN1,G1L, DG2, AGEN2, G2L, OC, ACOND, CL, DE, AE, EL,

$1 D A, A A B S, A L, A H X 1, A H X 2$

602 FORMAT $(1 X, * D G 1=*, F 5.4,5 X, * A G E N 1=*, F 4,1,5 X, * G 1 L=*, F 5.1,4 X, * 0 G 2=*$

$1, F 5.4,5 X, \cdot A G E N 2=$.

$1, F 4.1,5 X, * G 2 L=*, F 4.2, /, 1 X, * D C=*, F 5,4,6 X, * A C O N D=*, F 4,1,5 X$,

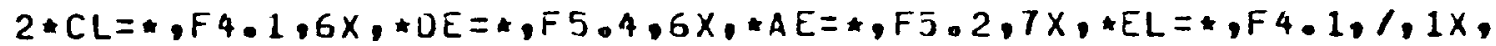

$3 * D A=*, F 5.4,6 X, * A A B S=*, F 4.1,6 X, * A L=*, F 5.1,5 X, * A H X 1=*, F 5.2$,

$44 \times, * A H \times 2=*, F 5.2 / 1$

PRINT 603,GM1,GM2,ABM, COY,CX(10), CX(1),CX(4)

603 FORMAT( $1 X_{*} * G M 1=*, F 5.1,5 X, * G M 2=*, F 5.1,6 X, * A B M=*, F 5.1,4 X, * C D M=*$,

$1 F 5.1,5 X, * C X(10)=*, F 4.3,4 X, * C X(1)=*, F 4.3,4 X, * C X(4)=*, F 4.3 /)$

PRINT $604, T(1), T(4), T(8), T(10), T(18), T G 2, T 20 T, T E P, T C P$,

1 IFF,FLH( 8$), F L H(10), F L 8, D P 1 B, E F F 1, E F F 2$

604 FORMAT $\left(1 X, * T(1)=*, F 5 \cdot 1,4 X, * T(4)=*, F 5 \cdot 1,5 X_{*} * T(8)=*, F 5.1,3 X_{0}\right.$

$1 * T(10)=*, F 5.1,3 X, * T(18)=*, F 5.1,4 X_{*} * T G 2=*, F 5.1,5 x_{0} * T 20 T=*, F 4.1$,

$25 X, * T E P=*, F 5.1,5 X, * T C P=*, F 5.1, /, 1 X, * I F F=*, I 1,9 X, * F L H 8=*, F 5.0$,

$35 X, * F L W 10=*, F 5 \cdot 0,2 X, * F L 8=*, F 5.0,5 X, * D P 18=*, F 4 \cdot 1,6 X, * E F F 1=*$,

$4 F 4.3,5 X_{0}+E F F 2= \pm, F 4.31$

PRINT 271

$L P C=0$

271 FORMATC1H1,3X,*TM TG

$1 * T(3) \quad T(4)$

2*T(7) T(8) T(10) T(11)*,//, TX, $T$ CX(1)

$3 * C \times(12) \quad P(1)$

TM TG1 TG2

$T(1)$

T(12) TE TEP TCH TCP*,
TE TCH

CX. (4) CX(8)

4* FLHÍ́ FLH2O CHC CHH CH*,8X,*FLHG*,1/,9X,*COP. QGENI*,

4* QGEN2 *,

5* OCONO OEVAP

QABS EFF 1

EFF2

$\operatorname{CDM}$

ABM $\quad 0 \times 20$

$0 \times 14 *, 11$ 
7 JUU $=J U U+1$

IF (JUU.EQ.ISTEPS) 31.253

31 CONTINUE

$J U=J U+1$

$Y(J U, 1)=$ QEVAP/120000.\$Y(JU,2)= DEVAP/QGEN 1

$X X X(J \cup)=T G 1$

$T G 1=T G 1-5$.

PRINT 20,TGI

20 FORMAT $\left(1 X_{,} * T G 1=*, F 10.3\right)$

JUU $=0$

253 CONTINUE

C

$C *$ ABSORBER PRESSURE* *

$T(20)=T 20 T$

$L C=0$ \& $N C=0$

C

C START OF ITERATIVE LOOPS

C

C BEGIN T(20) LOOP

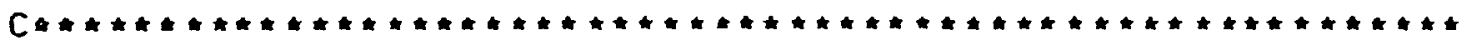

33 CONTINUE

$P(20)=V A P R(T(20))$

$P(19)=P(20)$

$P(8)=P(20)-.0000005 * F L W(18) * F L W(18)$

C LIMIT IMPOSED BY 32 DEGREE F FREEZING POINT

IF $(P(8) \cdot L T \ldots 08859) \quad P(8)=.08859$

$P(7)=P(8)$

C * * EVAPORATOR TEMPERATURE**

TC 19$)=T(20)$

$H(20)=H G T(T(20))$

$C X(12)=C X(10)$

C * ENTHALPY OF THE SOLUTION LEAVING THE ABSORBER

$H(10)=E N T H S O L(T(10), C X(10))$

$T 1 T=T(1) \& T(13)=T(1)$

$P(1)=$ PRESSOL $(T(1), E \times(1))$

$P(10)=P(11)=P(12)=P(13)=P(14)=P(2)=P(1)$

C FOR FIXED FLOW IFF $=0$, VARIABLE FLOH IFF $=1$

IF(IFF.NE. O) GO TO 35

$D E L P=P(1)-P(8)$

$F L H(10)=S Q R T(1000000 \cdot-2742.139 * D E L P \star D E L P)$

35 FLH(12) = FLW(10)

$H(1)=$ ENTHSOL $(T(1), C \times(1))$

$I=13$

CALL SUPER (P(I),T(1), 1, 1,S(I),H(I),V(I))

$T(14)=$ TVSP $(P(14))$

$T 14=T(14)$

* * SECOND GENERATOR ***

$36 P(4)=$ PRESSOL $(T(4), C \times(4))$

$P(3)=P(5)=P(15)=P(16)=P(17)=P(4)$

$T(17)=\operatorname{TVSP}(P(17))$

$H(17)=H F V S T(T(17))$

$H(19)=H(17)$

IF (IFLO.NE.1) GO TO 50

$F L W(13)=1.0 *((P(1)-P(4)) \cdot 144) *$.

$F L H(1)=F L H(12)-F L W(13)$

FLH(3) $=F L H(1)$ 
50 CONTINUE

$T(3)=$ TEMPSOL $(P(3), C \times(1))$

; $: 3$

IF(T (14),LE.T(3)) PRINT 301,T(14),T(3),T(4)

IF (T (14),LE.T (4)) PRINT 301,T(14),T(3),T(4)

301 FORMAT $(1 X, * T(14)=*, F 7.2$, IS LESS THAN T(3 OR 4),*,2F9.2)

IF(TC (14). GT.T(4)) GO TO 37

$T(4)=T(14)-.1$

C PREVENTS BAD LOG-MEAV TEMPERATURE CALCULATION

GO TO 36

37 CONTINUE

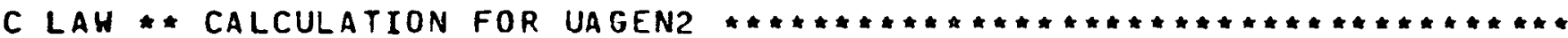

$G \times 2=(C X(1)+C \times(4)) / 2$.

HO2=HOGEN( GGEN2/AGEN2,P(4),GX2)

$L 13=N 13=0$

38 CONTINUE

GPP2 $=2 * F L H(13) /(G 2 L * G 2 N)$

HI 2 $=1 \cdot 51 *(4 \cdot * G P P 2 / Z M U(T(14))) *(-1 . / 3 \cdot) *((\operatorname{ZMU}(T(14))) * 2 \cdot / Z K W(T(1$

$(4)) * 3 .(Z R H O * * 2 .(417000000 \cdot)) * *(-1 . / 3$.

CONDG $2=0$.

UAGEN2 $=A G E N 2 /((A R / H I 2)+(1 \cdot / H O 2)+C O N D G 2)$

C LA A

QGEN2 = UAGEN2*(T(4)-T(3))/ALOG(T(14)-T(3))/(T(14)-T(4)))

IF (IFLO.NE.1) 60 TO 55

HI 4 MIN =HFVST $(T(3))$

$H(14)=H(15)=H(13)-Q G E N 2 / F L H(13)$

IF(H(14).LE.H14MIN) $H(14)=H 14 M I N$

H14=HFVST (T (14))

$S C=H 14-H(14)$

IF (SC.LE•Oค) SC $=0$.

C CP OF LIQUID WATER IS ASSUMED 1.

$T 14=T(14)-S C$

IF (T14.GT.T(3)) GOTO 40

$T 14=T(3)$

$H(14)=H(15)=H 14-(T(14)-T 14)$

QGEN2 $=F L H(13) *(H(13)-H(14))$

GO TO 40

$55 H(14)=H F V S T(T(14))$

$F L H(13)=Q G E N 2 /(H(13)-H(14))$

$F 13 C=F L H(13)$

CALL CONV(FLH(13),F 13C,2,N13,.0005)

$L 13=L 13+1$

IF(L13.GE.LIM) PRINT 245,L13

245 FORMAT $(1 X, *$ CONV HAS CALLED BY F13 LOOP*,I4, *TIMES*)

IF (LI3.GE.LIM) GO TO 999

IF (N13.NE.1) GO TO 38

$F L H(1)=F L W(12)-F L H(13)$

$F L H(3)=F L W(1)$

40 CONTINUE

$H(4)=$ ENTHSOL $(T(4), C \times(4))$

$I=16$

$T(16)=T(4)$

CALL SUPER (P(I),T(I), 1, 1,S(I),H(I),V(I))

$T(5)=T(4)-E F F 1 \cdot(T(4)-T(10))$

$H(7)=H(5)=E N T H S O L \quad(T(5), C X(4))$

$C X(7)=C X(5)=C X(4)$

$T(7)=$ TEMPSOL $(P(7), C X(7))$

$L H=0 \$ N H=0$ 


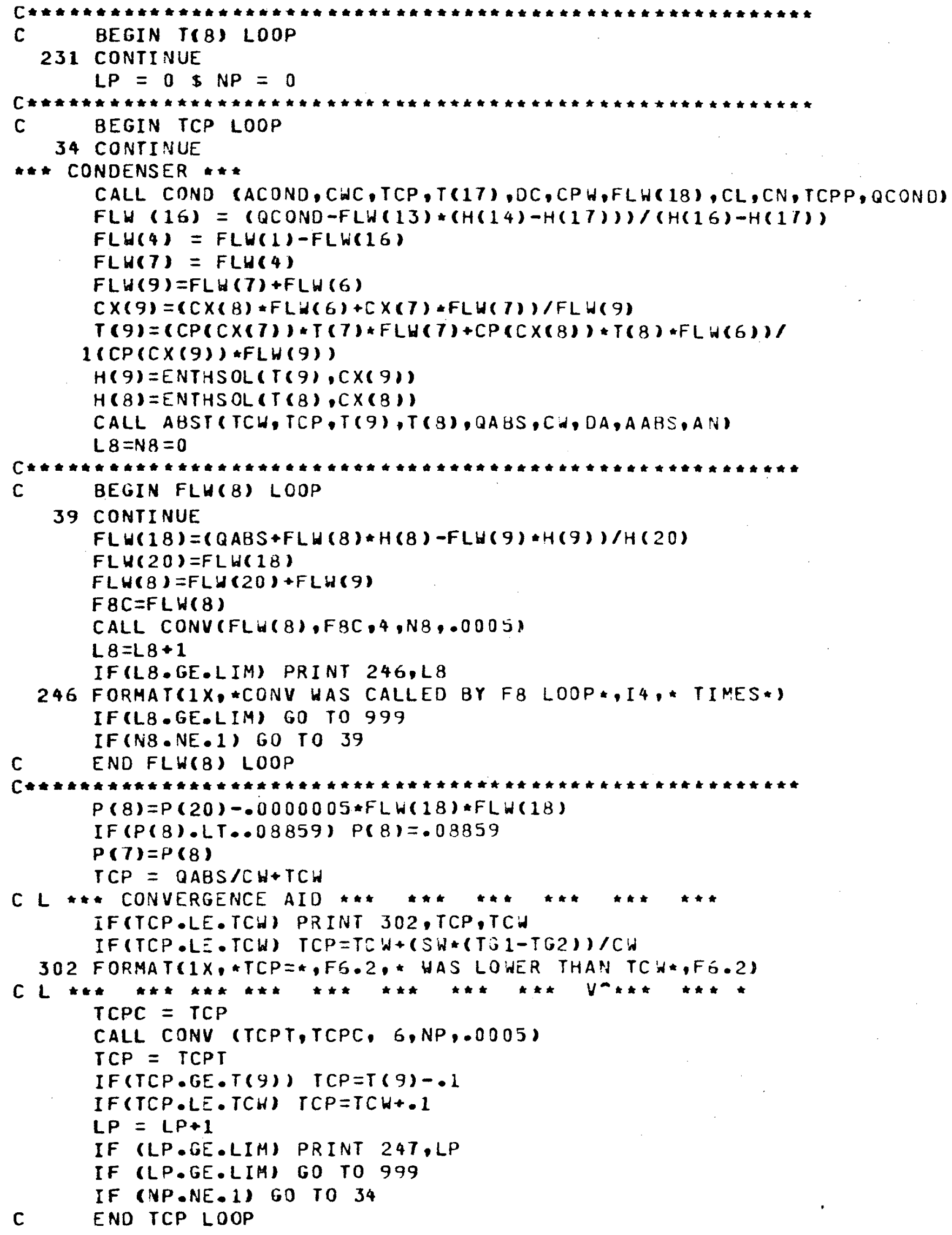




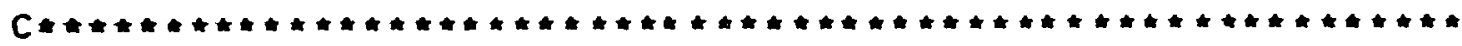

247 FORMAT (* CONV HAS CALLED BY TCP-LOOP*, I * TIMES*)

$C X(8)=F L H(9) * C X(9) / F L W(8)$

T $8 C=$ TEMPSOL $(P(8), C X(8))$

CALL CONV (T(B), T8C,5,NH.0.0005)

IF $(T(3) \bullet L E \bullet T C H) T(B)=T C H+\cdot 1$

LH $=L H+1$

IF (LH.GE•LIM)PRINT $250, L H$

IF (LH.GE.LIM) GO TO 999

IF (NH.NE.1) GO TO 231

C END T 8 LOOP

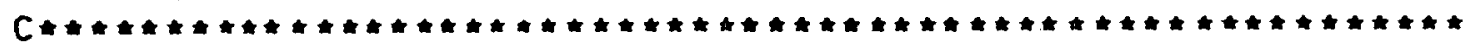

IF (TCT)-TCP . LT.0.0) PRINT 10,T(7).TCP

10 FORMAT (*TT,TCP *, 2FT.1)

250 FORHAT (* CONV HAS CALLED BY T8-LOOP*,I4, * TIMES*)

* HEAT EXCHANGERS **

$T(11)=T(10)+F \operatorname{L}(4) * C P(C X(4)) *(T(4)-T(5)) /(F L H(12) * C P(C X(12)))$

$T(2)=T(1)-E F F 2 *(T(1)-T(11))$

$H(3)=H(2)=E N T H S O L(T(2), C \times(1)$

$T(12)=T(11)+F L W(1) * C P(C X(1)) *(T(1)-T(2)) /(F L W(12) * C P(C X(12)))$

$H(12)=E N T H S O L(T(12), C \times(12))$

T12 = TEHPSOL $(P(1), C \times(10))$

C LAH * SECTION TO CALCULATE UAGENI

$G \times 1=(C \times(10)+C \times(1)) / 2$.

$A I=061 * 0 G 1 * P I / 4 . / A R / A R$

HO $1=H O G E N(Q G E N 1 / A G E N 1, P(1), G \times 1)$

$T G=(T G 1+T G 2) / 2$.

SUPT $=S W / G 1 N$

REI =DG 1/AR*SHPT/AI/ZMU(TG)

IF(RE L.LT.2300.) PRINT 280.REI

280 FORMAT(1X, ${ }^{\circ}$ RE OF GENL. IS NOT TURBULENT*,F10.0)

$P R=C P H * Z M U(T G) / Z K U(T G)$

$H I 1=.023 * R E 1 * \bullet 8 * P R *(.3) * 2 K H(T G) /(D G 1 / A R)$

CONDG $1=0$.

UAGEN1 =AGEN1/( (AR/HII)+(1-/HO1)+CONDG1)

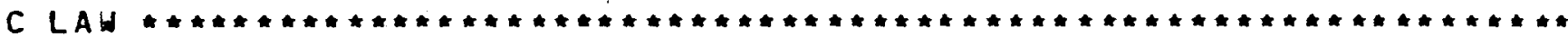

CALL GEN (UAGEN1,SH,TG1,TG2, T12,T(1),LL,LIM,1)

QGEN1 = SH*(TG1-TG2)

H(18) = HFVST $(T(18))$

C $L * * *$ NOTE IF FLH(18)=0---QEVAP=0 *** *** **

QEVAP $=F L W(18)+(H(20)-H(18))$

$T E P=T E+Q E V A P / C H H / C P H$

$T 2 O C=T(20)$

CALL EVAP (TE,TEP,T2OC,QEVAP,CHU,DE,AE,CPH,FLH(20),EL,EN)

CALL CONV (T2OT,T20C, 3,NC,.0005)

$\mathrm{T}(20)=\mathrm{T} 20 \mathrm{~T}$

$L C=L C+1$

IF (LC.GE.LIM) PRINT $446 . L C$

IF (LC.GE.LIM) GO TD 999

IF (NC.NE. 1) GOTO 33

C ENO T(20) LOOP

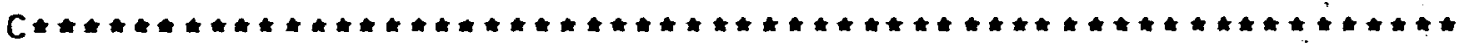

446 FORMATC CONY WAS CALLED BY T20 LOOP*I4** TIMES*) 
IF (T(5).LT.115คOR.T(T).LT.115.) GO TO 300

IF(T (4).LT.115.) GO TO 300

CALL C.RYSTAL (T(4),T(5),T(7),CX(4))

300 CONTI NUE

* * DEFINING QUANTITIES FOR PLOTTING * *

IF $((K J \cdot E Q \cdot 0) \cdot O R \cdot(K J \cdot E Q \cdot K C)) 255,257$

$255 J=J+1$

$X X Y(J)=T M$

$Y 1(J)=T(1)$ \& $Y 2(J)=T(4)$ \& $Y 3(J)=T(9)$

$Y 4(J)=C X(1) \& Y 5(J)=C X(4)$ \& $Y G(J)=C X(3)$

$K J=0$

$257 K J=K J+1$

HF $20=H F V S T(T(20))$

$Q X=(H(20)-H F 20) /(H G T(T(20))-H F 20)$

HF $14=$ HFVST $(T(14))$

HGT14 = HGT(T(14))

$$
\times 14=(H(14)-H F 14) /\left(H_{G T} 14 \quad-H F 14\right)
$$

COP $=$ QE VAP / QGEN 1

C

** derivative section * *

C

DABM $=F L H(8)-F L H(10)$

$D C D M=F L H(16)+F L H(13)-F L H(19)$

$D \times 1=(F L H(12) * C X(12)-F L H(1) * C X(1)) / G M 1$

$D \times 4=(F L W(1) * C X(1)-F L W(4) * C X(4)) / G M 2$

$D \times 10=(F L H(B) * C X(8)-F L H(10) * C \times(10)-C \times(10) * D A B M) / A B M$

$D T 1=C F L H(12) * H(12)-F L H(1) * H(1)-F L W(13) * H(13)+Q G E N 1+G M 1 * T(1): .7157$

$1 * D \times 1) / G M 1 / C P(C X(1))$

DT4 $=(F L H(3) * H(3)+Q G E N 2-F L$ W $(16) * H(16)-F L W(4) * H(4)+G M 2 * T(4) * .7157$

$1 * 0 \times 4) / G M 2 / C P(C \times(4))$

DT $10=(F L H(8) * H(8)-F L W(10) * H(10)-T(10) * C P(C X(10)) * D A B M+A B M * T(10) *$

$1 \quad .7157 * D \times 10) / A B M / C P(C \times(10))$

DT $18=($ F LW(13) +FLW(16)) *H(17)-FLH(18)*H(18)-T(18)*DCDM)/CDM

C

C * *TEST FOR PRINT AND FINISH**

C

CALL PRNTF CPRI,STR,FNR, NF, TG1, TG2,T14,TCPP,TE, TEP,TCH,TCP,SH, CHC 1, $C H H, C H, C O P, Q G E N 1, Q G E N 2, Q C O N D, Q E V A P, D A B S, E F F 1, E F F 2, C D M, A B M, Q X$, $2 \times 141$

C 60 TO $(5,8), N F$

C* INTEGRATION SECTION**

C

5 CALL INTI ( TM,DT.IO)

CALL INTG $(C \times(1), D \times 1)$

CALL INTG $(C \times(4), 0 \times 4)$

CALL INTG $(C \times(10), 0 \times 10)$

CALL INTG (T(1),DT1)

CALL INTS (T(4),DT4)

CALL INTG (T(10),OT10)

CALL INTG (T(18), OT18) 
IF $(T(18) \cdot G E \cdot T(17)) T(18)=T(17)$

CALL INTG (CDM,DCDM)

IF (CDM•GE•CDX) CDM=CDX

CALL INTG (ABM,DABM)

$E F F 1=E H X(A H \times 1,1)$

$E F F 2=E H \times(A H \times 2,2)$

GO TO 7

8 CONTINUE

NPTS $=J$

NCURVES $=3$

C

CALL CECPLOT (NCURVES, NPTS, XXY,Y $4, Y 5, Y 6$,

NCURVES $=3$

C

CALL CECPLOT (NCURVES, APTS,XXY,Y $1, Y 2, Y 3$ )

INC $=1$ S IY $=121 \$ M=2 \$$ IOPT $=1$

CALL USPLT $(X X X, Y, I Y, J U, M, I N C, \quad J A$, RG,ICHAR, IOPT, I MAG 4, IER )

$R G(3)=0$. $\$ \quad R G(4)=2$.

CALL USPLT $(X X X, Y, I Y, J U, M, I N C, J A, R G$, I CHAR, IDPT,I MAG4, IER )

IF (IER.EQ.65) PRINT 85

85 FORMAT (* H IS LT 1 *)

216 IF ( $(F L H(13), L E \cap 0.0) . O R \bullet(T(17) \cdot L E \cap T C P) . O R \cdot(T(4), L E . T(3)))$

1 PRINT 215,FLH(13),T(17), TCP,T (4),T(3)

215 FORMATC/* FL13 $=*$,F6.1/* T1J 1 *,F6.1,* TCP =*,F6.1/* T4=*,F6.1,

2* T3=* FG. 1 )

999 STOP \$ END 


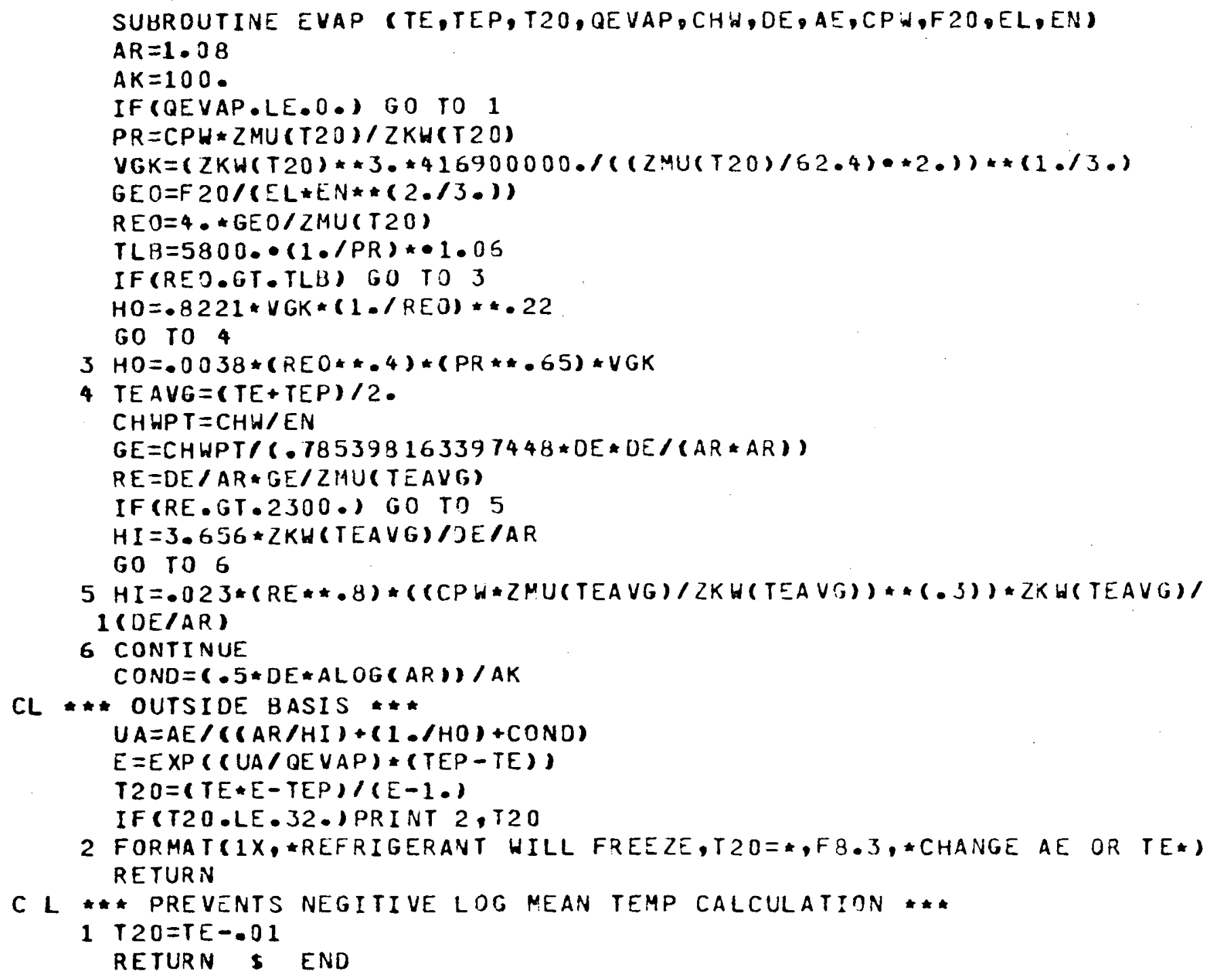


SUBROUTINE COND (ACOND,CHC,TCP,TIT,DC,CPH,FIS,CL,CN,TCPP,QCOND)

C FOR TURBULENT LIQUID FLOW INSIDE TUBES AND HEATING (PR**4)

$A R=1.08$

$A K=100$.

ZRHO $=61.9$

$T I C A=(T C P+T C P P) / 2$

$C H C P T=C H C / C N$

$G C=C * C P T /(.785338163397448 * D C * D C /(A R * A R))$

$H I=.023 *((D C / A R * G C / Z M U(T I C A)) * * 8) *((C P H * Z M U(T I C A) / Z K W(T I C A)$

$1) *(-4)) * Z K W(T[C A) /(D C / A R)$

$C O N D U C=(\cdot 5 * D C * A L O G(A R)) / A K$

C FOR CONDENSING ON THE OUTSIDE OF HORIZONTAL TUBES (LAMINAR)

$G P P C=F 18 /(C L *(C N *(2 . / 3 *)))$

$H O=1.51 *(4 * 6 P P C / Z M U(T 17)) *(-1.13) *.((Z K \forall(T 17) * 3) *(Z R H O * 2) * 417$

$1000000 . /(Z M U(T 17) * * 2)) *(1 . / 3$.

UACOND $=A C O N D /((A R / H I)+(1 \cdot / H O)+C O N D U C)$

$B=$ UACOND/CHC

$T C P P=T 17-(T 17-T C P) / E X P(B)$

QCOND $=$ CWC* $(T C P P-T C P)$

RETURN \$ END

$N C=0 \$ L L=0$

$A=$ UAGEN /SH 5 TT $=$ THO

$8 B=T H I-T C O-T T+T C I$

IF (THI.LE•TCO),OR・(TT•LE,TCI) PRINTS,THI,TT,TCI,TCO

C

9 FORMAT (* TG1,TG2,T12,T1:4F8.1)

AN ATTEMPT TO HELP CONVERGENCE

IF (TT - LE. TCI ) TTETCI+1.

$T C=T H I-A * B / A L O G((T H I-T C O) /(T T-T C I))$

CALL CONV (TT,TC, 1,NC,.0005)

$L L=L L+1$

IF (LL•GE•LIM) PRINT 14,I,LL

IF (LL.GE.LIM) 60 TO 16

IF (NC.NE. 1) GO TO 8

$T H O=T T$

14 FORMAT (* CONV HAS CALLED BY GEN *I1, I4, * TIMES*)

16 RETURN S END

FUNCTION CP(X)

$C P=.8752-.7157 * X$

RETURY \$ END

FUNCTION ENTHSOL (TI,XI)

$E_{1}=673.65 \$ E 2=-410.15 \$ E 3=-206.81 \$ E 4=45.0416$

$E 5=.8752 \$ E 6=-.7157 \ldots$

ENTHSOL $=X I *(X I *(X I * E I+E 2)+E 3)+E 4 *(E 5+E G * X I) *(T I-T T *)$

RETURN \$ END 
FUNCTION PRESSOL (TI,XI)

$A A 1=-17.86920 \$ A A 2=.880241 \$ A A 3=-.00776302$

$B B 1=25093.5599 \$ B B 2=-1026.057$ \$ $B B 3=9.16021$

$C C 1=-7844930 . \$ C C 2=282606.7$ \& $C_{1}=3=-2626.9764$

$X T=X I * 10^{*} 0$.

$T T=T I+459.67$

$A A=A A 1+(A A 2+A A 3 * X T) * X T$

$B B=B B 1+(B B 2+B B 3 * X T) * X T$

$C C=C C 2+(C C 2+C C 3 * X T) * X T$

$P L=A A+(B B+C C / T T) / T T$

$P R E S S O L=10 * * P L$

RETURN\$END

FUNCTION COMPSOL (TI,PI)

$A A 1=-17.86920 \$ A A 2=.880241 \$ A A 3=-.00796302$

$B B I=25093.5599 \$ B 32=-1025.057 \$ B B 3=9.15021$

$C C 1=-7844730 . \$ C C 2=282606.7$ b CC3 $=-2626.9764$

$T=T I+459.67$

$A A=T * T * A A 3+T * B B 3+C C 3$

$B B=T * T * A A 2+T * B B 2+C C 2$

$C C=T * T *(A A 1-A L O G 1 O(P I))+T * B B 1+C C I$

$X=(-3 B-S O R T(B B * B B-4 * A A * C C)) / 2 * / A A$

COMPSOL $=X / 100$.

RETURN \$ END

FUNCTION TEMPSOL (PI, XI)

$A A 1=-17.86920 \$ A A 2=.880241$ \$AA3 $=-.00796302$

$B B 1=25093.5599 \$ B 92=-1026.057 \$ B B 3=9.16021$

$C C_{1}=-7844930 . \& C=2=282606.7$ \& CC3 $=-2626.9764$

$X T=X I .100$.

$A A=A A 1+(A A 2+A A 3 * X T) * X T$

$B B=B B 1+(B B 2+B B 3 * X T) * X T$

$C C=C C 1+(C C 2+C C 3+X T) * X T$

$A L P=A L O G 10(P I)-A A$

$T T=(B B-S Q R T \quad B B \star 2 * 4 * A L P \star C C)) /(2 \star A L P)$

TEMPSOL $=T T-459.67$

RETURN \$ END

FUNCTION TH2C (HI)

$T H 20=31.93785+1.00081138 * H I$

RETURN \$ END

FUNCTION TSOL (HI, XI)

$E_{1}=673.55 \mathrm{~B} E 2=-410.15 \$ E 3=-205.91 \$ E 4=45.0416$

$E 5=.8752 \$ E 6=-.7157$

TSOL $=(H I-X I *(X I *(X I * E 1+E 2)+E 3)-E 4),(E 5+E 6 * X I)+77$.

RETURN D END 
FUNCTION TUSP(P)

C..-DETERMIVATION OF THE SATURATION TEMPERATURE OF WATER, GIVEN PRESSURE DIMENSION A(B)

C... DETERMINE PRESSURE RANGE --

IF $(P-450) 1,$.

C ... COMPUTE TEMPERATURE FOP PRESSURE. IN LOW RANGE --

$1 A O=35.157890$ \$ $A(1)=24.592588$ \& $A(2)=2.1182069$ \& $A(3)=-0.3414474 J$

$A(4)=0.15741542 \& A(5)=-0.031329585$ \& $A(6)=0.0038658282$

$A(7)=-0.00024901784$ \& $A(8)=0.0000068401559$

$X=A L O G(10 * P)$

TVSP $=A O+A(1) \star X$

DO $10 \quad I=2,8$

10 TVSP $=$ TVSP+A(I)*X*\#

RETURN

C -.. COMPUTE TEMPERATURE FOR PRESSURE I: HIGH QANGE --

$2 A O=11545.164$ \& $A(1)=-8386.0182$ \& $A(2)=2477.7661$ \& $A(3)=-363.44271$ $A(4)=25.690978$ \& $A(5)=-0.73073813$ \& $X=A \operatorname{LOG}(P)$ \& $T V S P=A O+A(1) * X$ DO $11 \mathrm{I}=2.5$

11 TVSP $=T V S P+A(I) \bullet X * * I$

RETURN \& END

FUNCTION HFVST (T)

C DETERMINATION OF THE ENTHALPY OF HATER AT THE SATURATED LIOUID

C CONDITION, GIVEST TEMPERATURE

DIMENSION A(5)

C... DETERMINE TEMPERATURE RANGE --

IF $(T-360,1,2,2$

C -.. SET CONSTANTS FOR LOH RANGE --

$1 A O=-32.179105$ S $A(1)=1.0088034$ S $A(2)=-1.1516996 E-4$

$A(3)=4.855836 E-7 \$ A(4)=-7.3613778 E-10 \$ A(5)=9.6350315 E-13$

GO TO 100

C -.. SET CONSTANTS FOR HIGH RANGE --

$2 A D=-904.11706 \& A(1)=10.673802$ \& $A(2)=-4.2753336 E-2$

$A(3)=9.41244 E-5$ \& $A(4)=-1.0315357 E-7$ \& $A(5)=4.560246 E-11$

C... COMPUTE ENTHALPY --

$100 \mathrm{HFVST}=A D+A(1) * T$

DO $10 \quad I=2,5$

10 HFYST $=$ HFVST+A(I) $\bullet T \bullet \cdot I$

RETURN \$ END

FUNCTION ZKW(T)

CL *** FUNCTION TO FIND THE THERNMAL CONDUCTIVITY CF

CL SATURATED LIQUID WATER

IF(T.LT.32_.OR.T.GT.400.) PRIINT 1,T

ZKW $=-.0000012 * T * T+.000644 * T+.3099633$

1 FCRMAT(1X,FG.2, IS JUTSIDE THE RANGE FOR ZKH*)

RETURN \& END

FUNCTION ZMU(T)

CL *** FUNCTION TO FIND VISCOSITY JF SATURATED WATER AS A

CL FUNCTION OF TEMPERATURE

IF (T.LT.32,.CR.T.GT.350.) PRINT 1,T

ZMU $=211.4363177 *(1 . /(T * 1.068252605))$

1 FORMAT(1X,F6.2, IS OUTSIDE THE RANGE FOR ZMU*)

RETURN \$ END 


\section{FUNCTION TVSPH $(P, H)$}

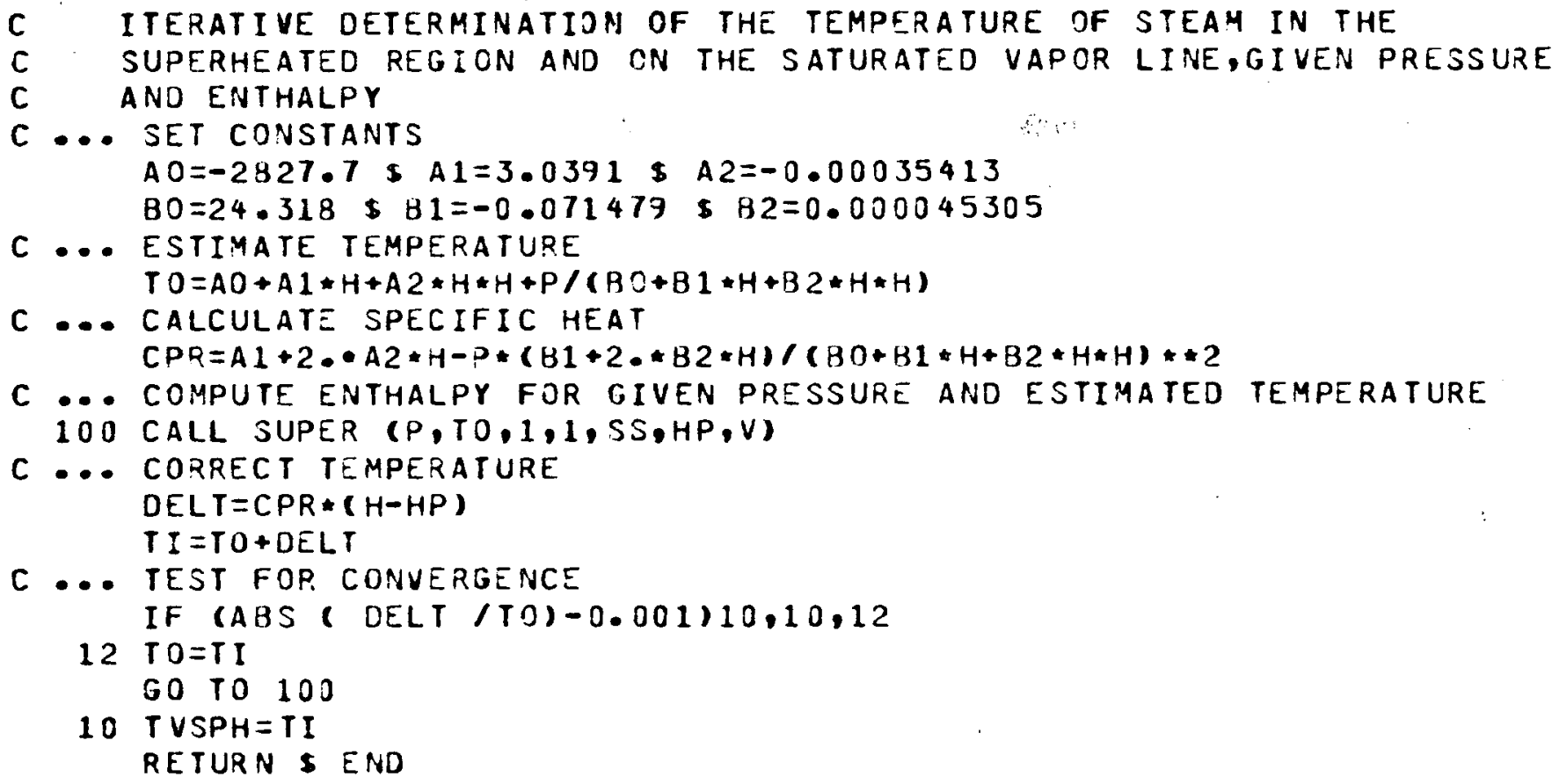

1 FORMAT $(1 X, * T 4,(*, F 6.2, *)$ IS GREATER THAN THE CRYSTALIZATION 1 LIMIT $(*, F 6.2, *), C X(4)=\star, F 5.3)$

2 FORMAT(1X,*T5,(*,F6.2,*) IS GREATER THAN THE CRYSTALIZATION 1 LIMIT $(*, F 6.2, *), C X(4)=*, F 5.3)$

3 FORMAT $(1 X, * T 7(*, F 6.2, *)$ IS GREATER THAN THE CRYSTALIZATION

1 LIMIT(*,F6.2,*),CX(4)=*,F5.3)

RETURN \$ END

FUNCTION HOGEN(Q,P,X) $H 25=E X P(.795 * A L O G(0)-.132249348 * A L O G(P)-.73381733)$

IF $(X \cdot B E \cdot .25)$ GO TO 25 $H 10=E X P(.78 * A L O G(1)-.158899639 * A L O G(P)-.401968398)$ $H O G E N=H 25-((.25-X) \bullet(H 25-H 10)) / .15$

GO TO 99

$25 H 5 C=E X P(.66 * A L O G(0)-.162354479 * A L O G(P)+.492493016)$ HOGEN $=((X-.25) *(H 50-H 25)) / .25 * H 25$

99 CONTINUE

RETURN \$ ENO 


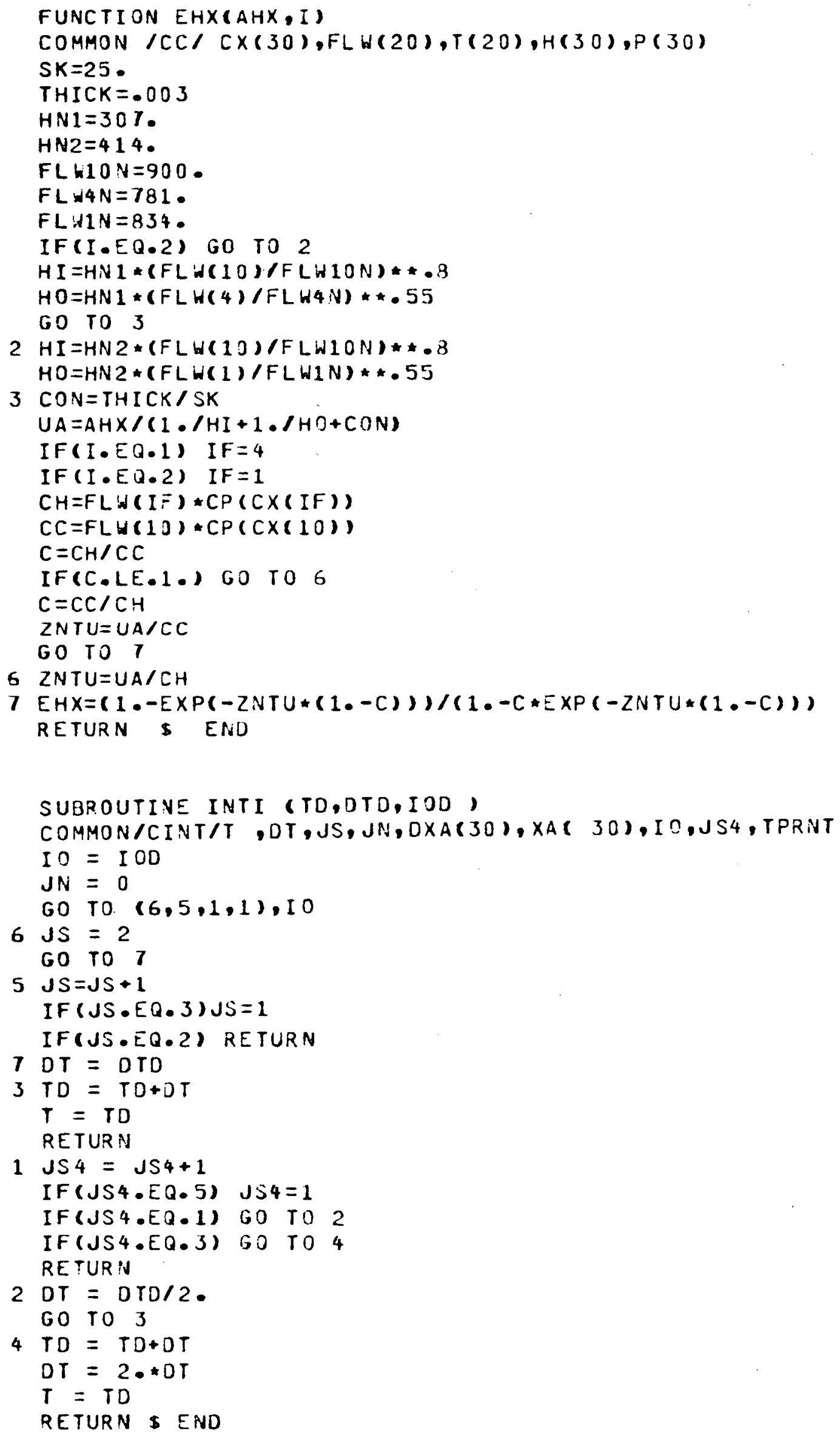




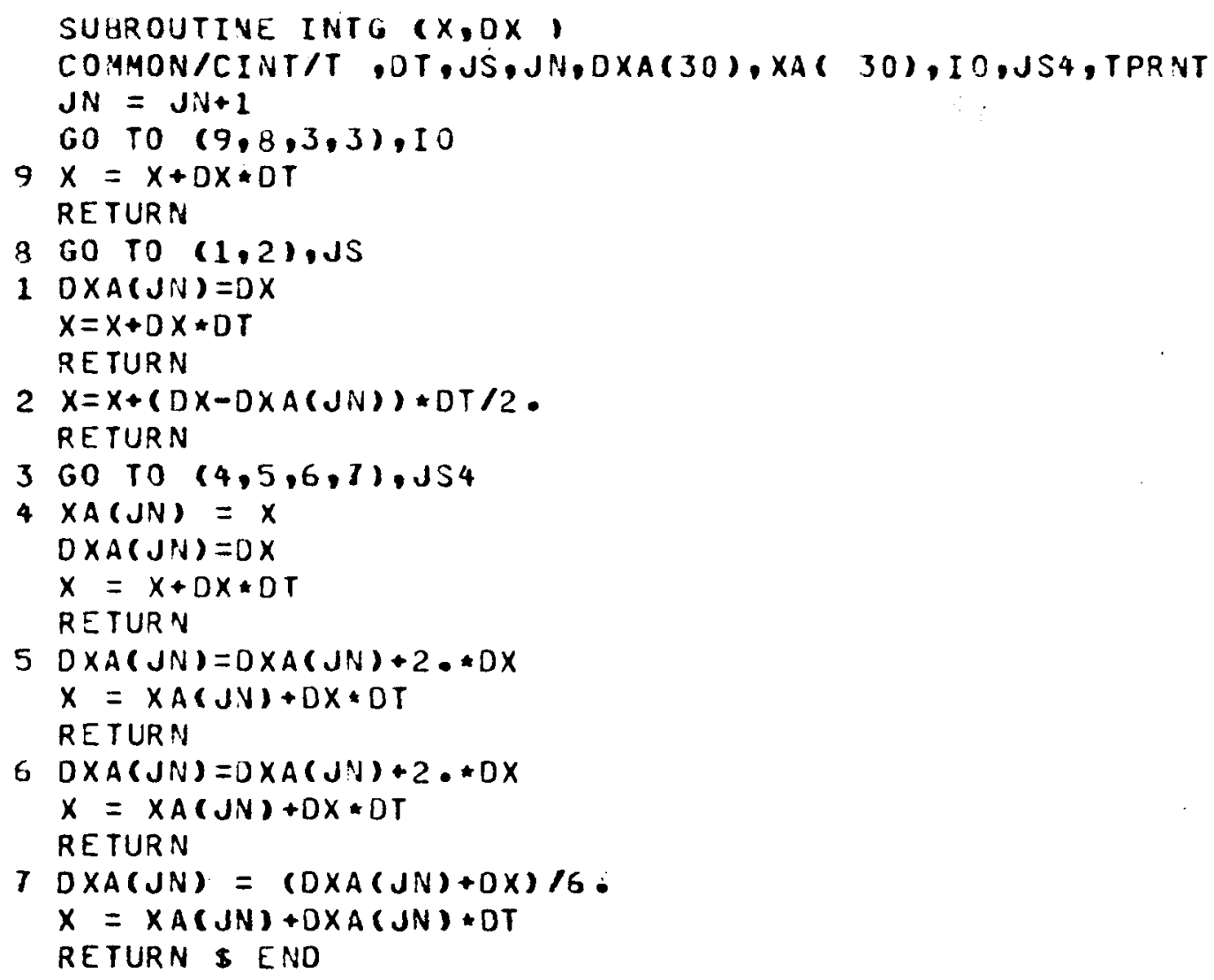


SUBROUTINE PRNTFCPRI, STR, FNR, NF, TGI,TG2,T14,TCPP, TE,TEP,TCW, ITCP,SH,CHC,CHH,CH, COP, OGEN1, JGEN2, QCOND, QEVAP, QABS, EFF1, EFF2, $2 C D M, A B M, Q X, X 14)$

COMMON/CC/CX(30), FLW(20), T(20),H(30),P(30)

COMMON/CINT/TM,DT,JS,JU,OXA(30),XA( 301, IO,JS4, TPRNT

100 F ORMATC $/, 1 X, F 5.2,2 X, F 5.1,5 F B .1,1 X, F 5.1,2 F 7.1,2 F 5.1,1 X$,

$13 F 6.1,1 X, 3 F G .1,2 F 5.0,1,4 X, 4 F 8,3, F 8,2, F 8,3, F 6,3,2 F 7.1, F 6.1$,

$2 F 6.0, F 7.1,2 F G .1, F 7.0, F 6.0, F 7.0, F 9.1, /, 7 X, F 5.3,5 F 8.0, F 6.3, F 7.3,1 X$,

$32 F 6.1, F 6.2, F 7.2)$

IF (TPRNT.LT.PRI) GO TO 4

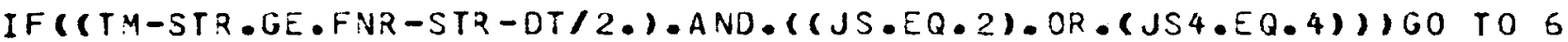

IF ( (TM-STR.GE.TPRNT-DT/2.).AND. ( (JS.EQ.2).OR・(JS4.EQ.4)) GO TO J

$I=I N T(T M)$

$A=T M-F L O A T(I)$

$B=1 \cdot-2 \cdot * D T$

$C=A-B$

$D=A B S(C)$

IF (D.LT.DT/2, ) GO TO 8

RETURN

$4 \mathrm{NF}=1$

5 TPRNT $=$ TPRNT +PRI

3 PRINT 100,TM,TG1,TG2,T(1),T(12),T(2),T(3),T(4),T14,T(17),TCPP,

$1 T(20), T E, T E P, T C W, T C P, T(7), T(8), T(10), T(11), C X(1), C X(4), C X(8)$,

$2 C X(12), P(1), P(4), P(20), F L W(12), F L W(1), F L H(13), S$ S,$F L H(4)$,

3FL

4, EFF1, EFF $2, C D M, A B M, Q X, X 14$

IF $(T M \cdot E Q \cdot 0 \bullet I L C=0$

$L P C=L P C+1$

IF(LPC.NE - 12) GO TO 9

PRINT 271

271 FORMATC1H1,3X,*TM TG

2* TCP T(T) T(8) T(10) T(11)*,//,7X,*CX(1)

$3, * C X(8) \quad C X(12) \quad P(1) \quad P(4) \quad P(20) \quad F L H 12$ FLH1 FLH13*,

4* SH FLH4 FLH1S FLW2O CHC CHW CW*,8X, \#LHG*,//,

$59 X, * C O P$ QGEN1 GGEN2 BCOND QEVAP QABS EFF1*,

6* EFF2 CDM ABM QX20 QX14*1

$\angle P C=0$

9 CONTINUE

RETURN

- $T M=F N R$

TPRNT $=0.0$

$N F=2$

DO $7 J=1,30$

$7 \times A(J)=0.0$

GO TO 8

END 
APPENDIX B

HEAT TRANSFER COEFFICIENTS FOR WATER-LITHIUM BROMIDE

SOLUTIONS AS FUNCTIONS OF HEAT FLUX,

PRESSURE, AND CONCENTRATION

(Data from Minchenko and Firsova [10]) 


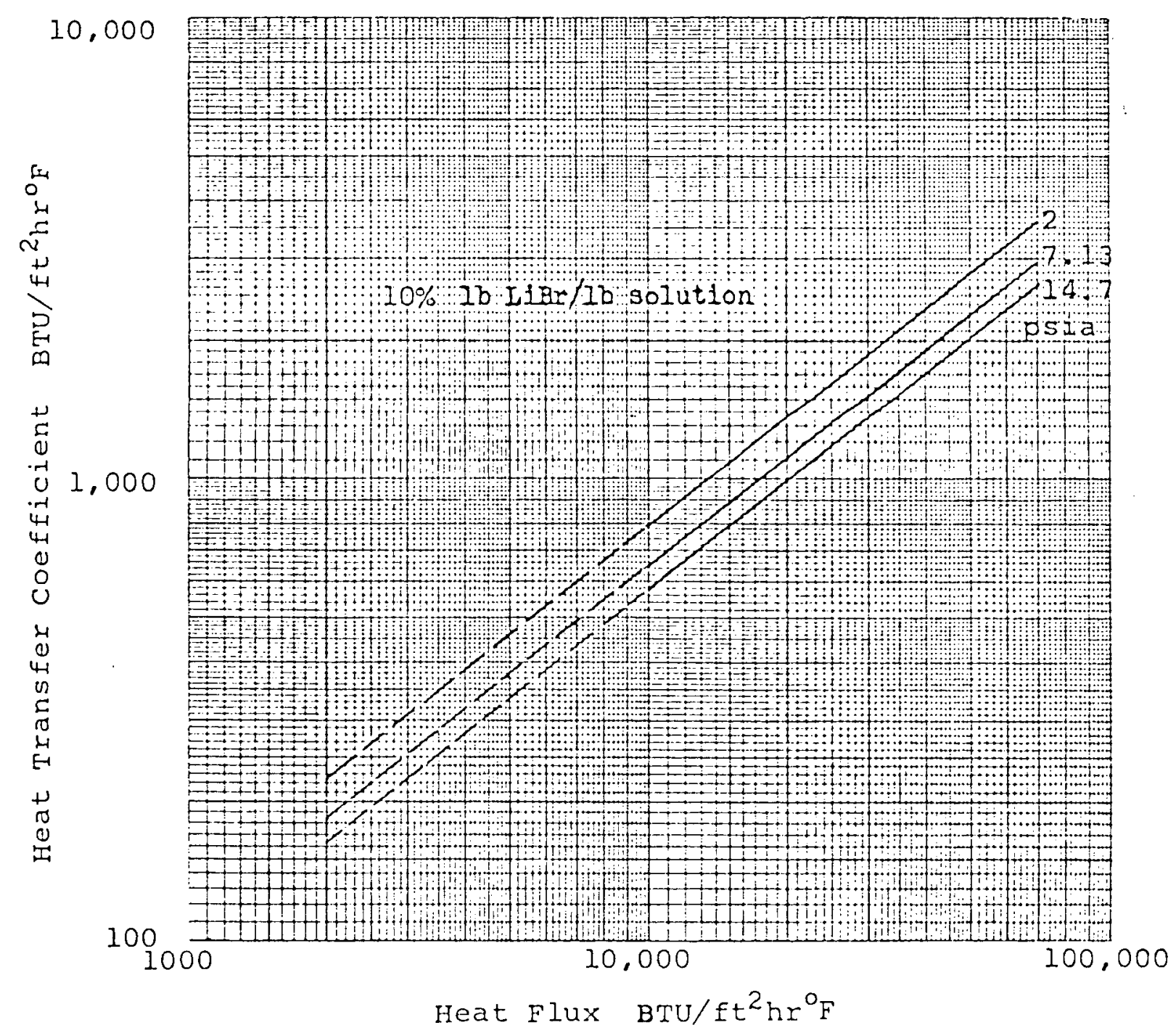




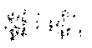
61:

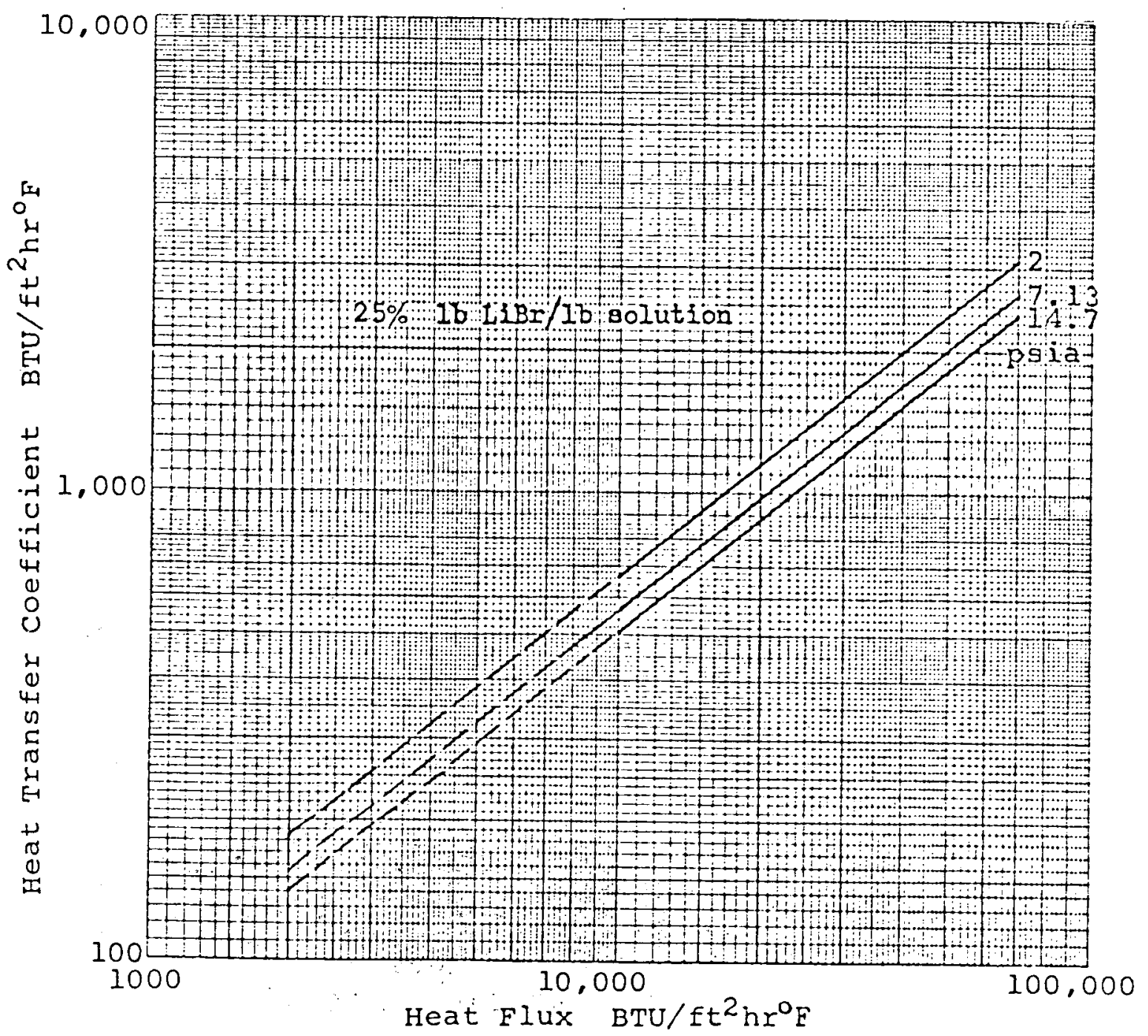




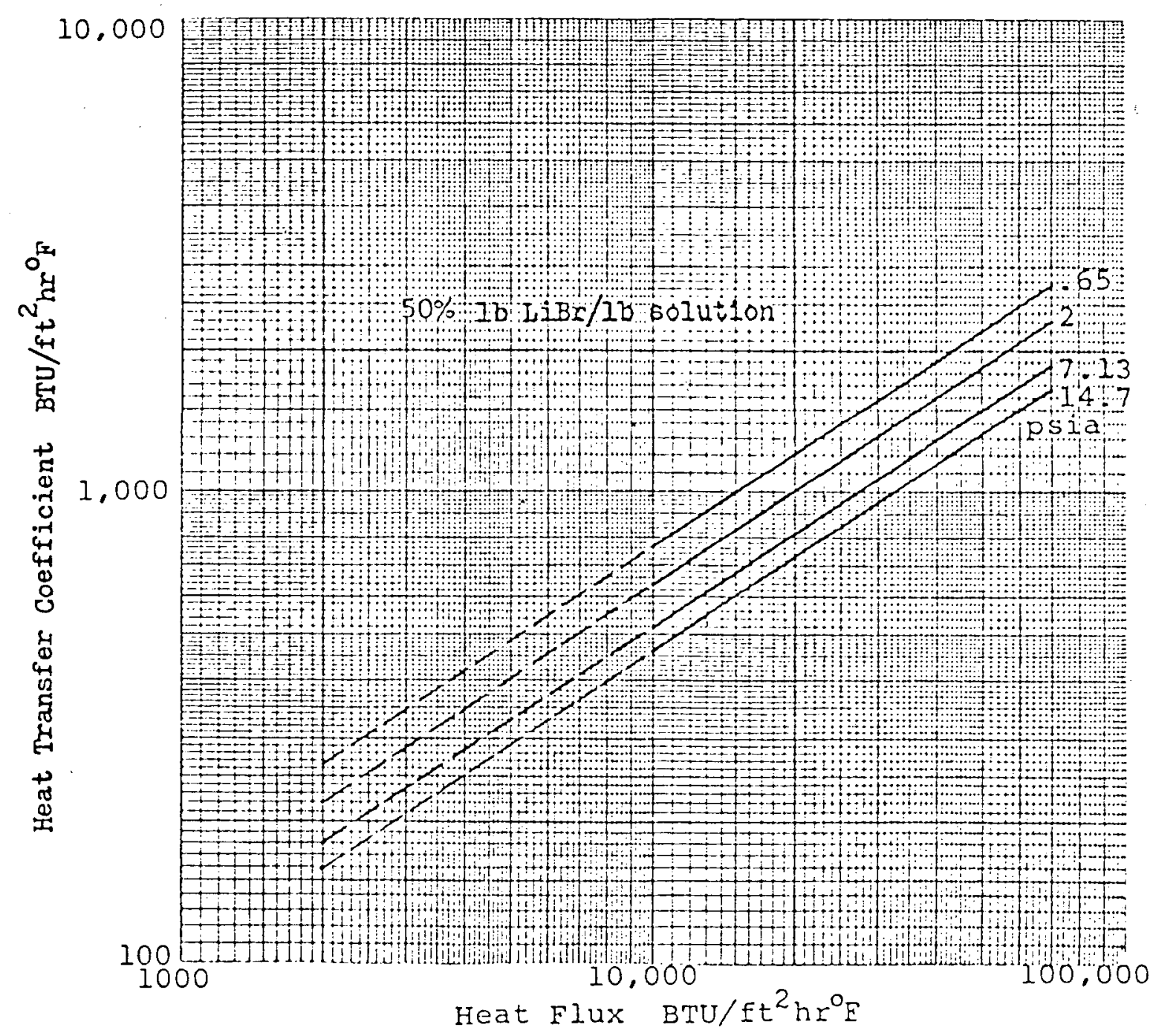




$$
4
$$

APPENDIX C

EQUILIBRIUM CHART FOR WATER-LITHIUM

BROMIDE SOLUTIONS

(From ASHRAE Handbook of Fundamentals [22]) 


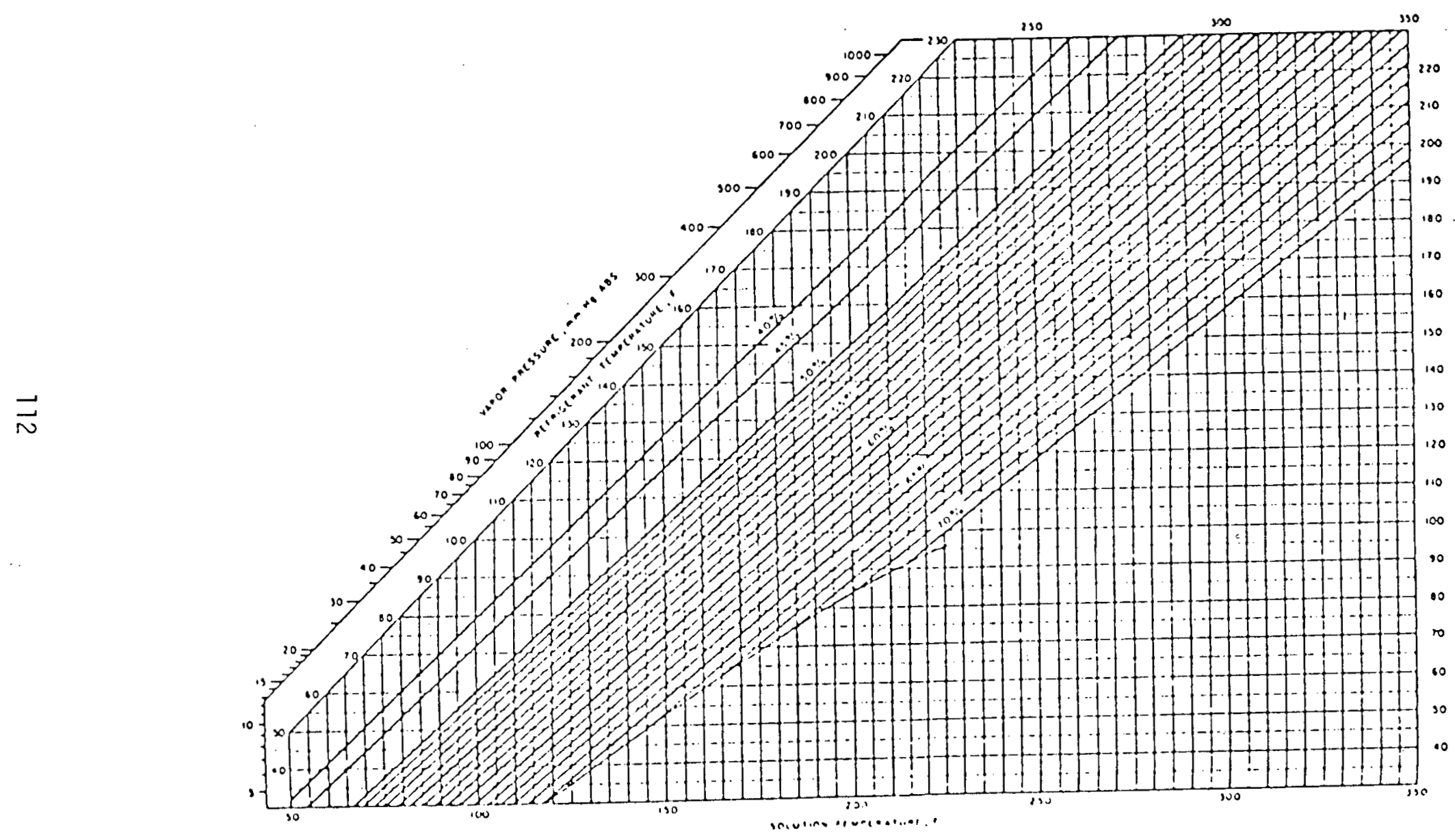




$$
\text { in }
$$

$\cdots$

Appendix D

THE NOMINAL CONDITION 
Source hot water temperature

Cooling water temperature

Chilled water temperature

Source hot water flow rate

Cooling water flow rate

Chilled water flow rate

Circulation flow rate

Generator 1 Area

Generator 2 Area

Condenser area

Evaporator area

Absorber area

Heat exchanger 1 area

Heat exchanger 2 area

Tube size

Area ratio

Evaporator to absorber pressure drop coefficient
$\left(\mathrm{T}_{\mathrm{s}}\right)$

$\left(T_{C}\right)$

$\left(T_{c h}\right)$

$\left(m_{s}\right)$

$\left(m_{c}\right)$

$\left(m_{c h}\right)$

$[\mathrm{m}(10)]$

$\left(A_{G 1}\right)$

$\left(A_{G 2}\right)$

$\left(A_{C}\right)$

$\left(A_{E}\right)$

$\left(A_{A}\right)$

$\left(A_{H X\rceil}\right)$

$\left(A_{H \times 2}\right)$

(AR)

(c) $280^{\circ} \mathrm{F}$

$85^{\circ} \mathrm{F}$

$44^{\circ} \mathrm{F}$

5,000 1b/hr

$18,000 \mathrm{~Tb} / \mathrm{hr}$

$10,000 \mathrm{lb} / \mathrm{hr}$

$900 \mathrm{lb} / \mathrm{hr}$

$35 \mathrm{ft}^{2}$

$20 \mathrm{ft}^{2}$

$20 \mathrm{ft}^{2}$

$55 \mathrm{ft}^{2}$

$60 f t^{2}$

$5 f t^{2}$

$5 \mathrm{ft} t^{2}$

0.5 in

1.08

$5 \times 10^{-7} \frac{1 b f-h r^{2}}{i n^{2}-1 b^{2}}$ 


\section{REFERENCES}

1. Daniels, Farrington, Direct Use of the Sun's Energy, Ballantine Books, New York, 1974.

2. Auh, Paul C., A Survey of Absorption Cooling Technology in Solar Applications, Brookhaven National Laboratory Report Number BNL50704, Upton, New York, July 1977.

3. Whitlow, E. P., and Swearingen, E. P., "An Improved Absorption Refrigerant Cycle," J. Gas Age, October 1958.

4. Versagi, Frank J., Technical Conversations in Air Conditioning and Refrigeration, Business News Publishing Company, Detroit, 1962.

5. Vliet, Gary C., and Saiidi, Mohammad J., "Double Effect Absorption Cooling with Solar Energy," Proceedings of the Third Workshop on the Use of Solar Energy for Cooling of Buildings, 1978.

6. Lithgow, Rodolfo A., Preliminary program and documentation and personal communications between June 1979 and August 1980.

7. Franks, Roger G. E., Modeling and Simulation in Chemical Engineering, Wiley-Interscience, New York, 1972. 
8. Himmelblau, David M., and Bischoff, Kenneth B., Process Analysis and Simulation: Deterministic Systems, John Wiley and Sons, Inc., New York, 1968.

9. Holman, J. P., Heat Transfer, First Edition, McGraw-Hill Book Company, New York, 1972.

10. Minchenko, F. P., and Firsova, E. V., "Heat Transfer to Water and Water-Lithium Salt Solutions in Nucleate Pool Boiling," Problems of Heat Transfer and Hydraulics to Two Phase Media, Edited by S. S. Kutateladze, Pergamon Press, New York, 1969.

11. Ellington, R. T., et al., "The Absorption Cooling Process," Institute of Gas Technology, Research Bulletin 14, 1957.

12. Kern, Donald Q., Process Heat Transfer, McGraw-Hill Book Company, New York, 1950.

13. Chun, K. R., and Seban, R. A., "Heat Transfer to Evaporating Liquid Films," ASME Journal of Heat Transfer, November 1971.

14. Merrick, Richard, ARKLA Industries, personal communications, May 1980.

15. The Trane Company, plant visit, February 15, 1980. 
16. Kays, W. M., and London, A. L., Compact Heat Exchangers, McGrawHill Book Company, New York, 1964.

17. Lawson, B. M., "A Parametric Analysis of Water-Lithium Bromide Double-Effect Absorption Cooling Systems," M.S. Thesis, The University of Texas at Austin, December 1980.

18. The Trane Company, "Two-Stage Absorption Cold Generator," 1979, Service Bulletin ABS-SB-35, November 1978.

19. Personal communication with Mr. L. Porter of the Trane Company, spring 1980.

20. The Trane Company, "Technical Data: Two-Stage Absorption Cold Generator", Brochure D-ABS2-20-1072-175-DJL-RFS.

21. The Trane Company, "Two-Stage Absorption Cold Generator," Absorption/ Refrigeration Brochure D-ABS-2/Feb 1979.

22. ASHRAE, ASHRAE Handbook of Fundamentals, American Society of Heating, Refrigerating, and Air Conditioning Engineering, New York, 1977.

23. Uemura, Tadashi, and Hasaba, Shigeo, "Studies on the Lithium Bromide-Water Absorption Refrigerating Machine," Technology Reports of Kansai University No. 6, Osaka, Japan, December 1964. 
24. Himmelblau, David M., Applied Nonl inear Programming, McGraw-Hill Book Company, New York, 1972.

25. Ward, Dan S., "Solar Absorption Cooling Feasibility," Solar Energy, Vo1. 22, Pergamon Press Ltd, Great Britain, 1979. 